\title{
Site-Specific Health and Safety Plan for Work Activities at the Natural and Accelerated Bioremediation Research (NABIR) Field Research Center (FRC), Oak Ridge, Tennessee
}

\author{
D. B. Watson \\ H. D. Quarles
}


Environmental Sciences Division

\title{
Site-Specific Health and Safety Plan for Work Activities at the Natural and Accelerated Bioremediation Research (NABIR) \\ Field Research Center (FRC), Oak Ridge, Tennessee
}

\author{
D. B. Watson \\ Environmental Sciences Division \\ Oak Ridge National Laboratory \\ Field Research Center Manager \\ H. D. Quarles \\ Environmental Sciences Division \\ Oak Ridge National Laboratory \\ Field Research Center Regulatory Specialist
}

August 2000

Prepared by

Environmental Sciences Division

Oak Ridge National Laboratory

UT-Battelle, LLC.

ESD Publication 5040

Prepared for

U.S. Department of Energy

Office of Environmental Restoration and Waste Management

Under budget and reporting code EW 264502000

\author{
OAK RIDGE NATIONAL LABORATORY \\ Oak Ridge, Tennessee 37831-6285 \\ managed by \\ UT-BATTELLE, LLC \\ for the U.S. Department of Energy \\ under contract number DE-AC05-00OR22725
}


Greg Rowlond

Greg Rowland

ORNL HAZWOPER Program Coordinator $\frac{8 / 30 / 00}{\text { Date }}$

$\frac{8 / 30 / 00}{\text { Date }}$
Mary Annd Bogle

Site Safety and Health Officer

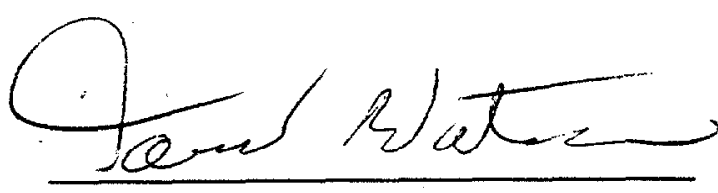

David B. Watson

$\frac{8 / 24 / 00}{\text { Date }}$ 


\section{CONTENTS}

ACRONYMS

FORWARD

1. INTRODUCTION AND PROJECT DESCRIPTION ………………………………………….. I

1.1. INTRODUCTION

1.2. DESCRIPTION OF ACTIVITIES CONDUCTED ON THE FRC ……………………...... 3

2. SITE ORGANIZATION AND COORDINATION ......................................................

2.1. SITE SAFETY AND HEALTH OFFICER ……………..........................................5

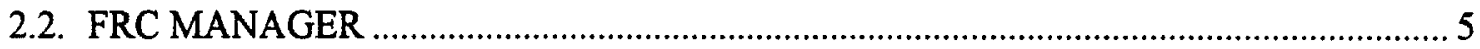

2.3. PRINCIPAL INVESTIGATORS AND FIELD PROJECT PERSONNEL .......................... 6

2.4. RADIATION PROTECTION ………………………...........................................

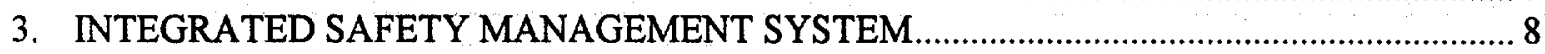

4. TASK SPECIFIC HAZARD EVALUATION AND CONTROLS ……………………............9

4.1. FIELD SAMPLING AND ANALYSIS OF GROUNDWATER ………………….........9

4.2. TRACER TESTS OR MICROBIAL MANIPULATION AND TRANSPORT

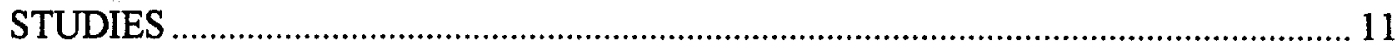

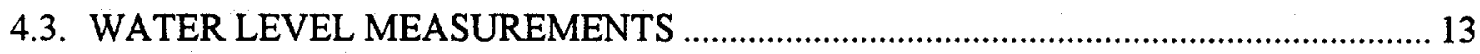

4.4. HYDRAULIC TESTING (E.G., PUMPING TESTS, SLUG TESTS, POINT DILLUTION TESTS AND FLOW METERS SUCH AS THE COLLOIDAL BORESCOPE).

4.5. CORING AND WELL INSTALLATION............................................................... 16

5. OTHER HEALTH AND SAFETY PLAN ELEMENTS …………………………………..... 20

5.1. REVISIONS/ MODIFICATIONS TO THE HASP ....................................................... 20

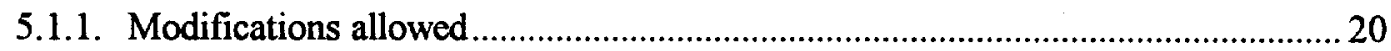

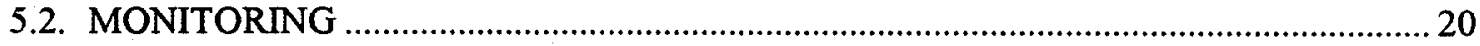

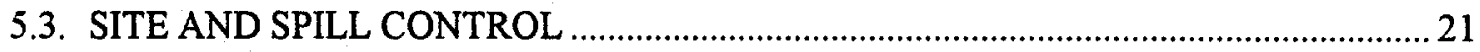

5.4. PERSONAL PROTECTIVE EQUIPMENT ………................................................... 21

5.5. TEMPERATURE EXTREMES AND SITE CHARACTERISTICS ………………........21

5.5.1. Effects and Prevention of Heat Stress.............................................................2

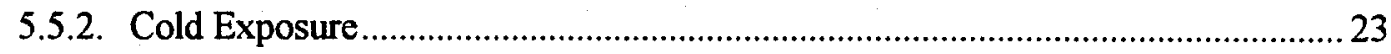

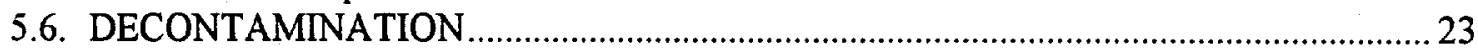

5.7. EMERGENCY PREPAREDNESS/RESPONSE ................................................. 24

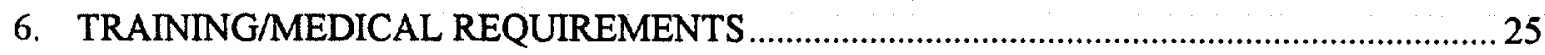

6.1. SITE-SPECIFIC HAZARD COMMUNICATION AND ACCESS BRIEFING ……….....25

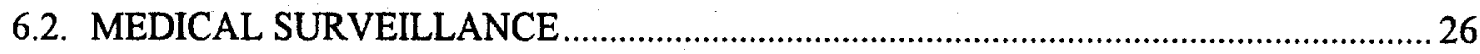

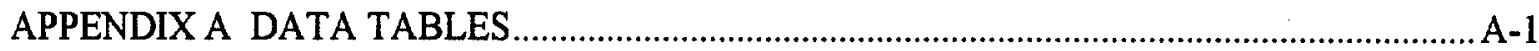

APPENDIX B MATERIAL SAFETY DATA SHEETS …………............................................ B-1

APPENDIX C HEALTH AND SAFETY PLAN ACCEPTANCE AND TRAINING ACKNOWLEDGEMENT …………..................................... C-1 


\section{ACRONYMS}

$\begin{array}{ll}\text { BY/BY } & \text { Bone Yard/Burn Yard } \\ \text { CFR } & \text { Code of Federal Regulations } \\ \text { DOE } & \text { Department of Energy } \\ \text { ESD } & \text { Environmental Sciences Division } \\ \text { ESH\&Q } & \text { Environmental Safety, Health, and Quality } \\ \text { FRC } & \text { Field Research Center } \\ \text { ISMS } & \text { Integrated Safety Management Systems } \\ \text { HASP } & \text { Health and Safety Plan } \\ \text { LSS } & \text { Laboratory Shift Superintendent } \\ \text { MSDS } & \text { Material Safety Data Sheet } \\ \text { NABIR } & \text { Natural and Accelerated Biorcmediation Research } \\ \text { ORNL } & \text { Oak Ridge National Laboratory } \\ \text { ORR } & \text { Oak Ridge Reservation } \\ \text { OSHA } & \text { Occupational Safety and Health Act } \\ \text { PPE } & \text { Personal Protective Equipment } \\ \text { PSS } & \text { Plant Shift Superintendent } \\ \text { RWP } & \text { Radiological Work Permit } \\ \text { SSHO } & \text { Site Safety \& Health Officer } \\ \text { VOC } & \text { volatile organic compound } \\ \text { WSS } & \text { Work Smart Standards }\end{array}$




\section{FOREWORD}

The Occupational Safety and Health Act (OSHA) implementing regulations of 29 CFR 1910.120 govern hazardous waste operations and emergency response. These regulations require that employers of employees involved in certain specific hazardous waste operations 1) develop and implement a written safety and health PROGRAM for employees involved in hazardous waste operations, and 2) that the PROGRAM incorporate a site-specific safety and health plan.

Oak Ridge National Laboratory (ORNL) has employees conducting activities which fall within the scope of these regulations, and thus, has in place a written safety and health PROGRAM as required. Its contents are contained in the ORNL HAZWOPER Program Manual. Some activities conducted at the contaminated portion of the Natural and Accelerated Bioremediation Research Program's Field Research Center may fall within the scope of these OSHA regulations. Thus, to assure regulatory compliance, this site-specific safety and health plan covering activities conducted at the contaminated portion of the Field Research Center has been prepared. The Integrated Safety Management System and Environmental Safety, Health, and Quality check lists will be used to define safe work procedures for work conducted in uncontaminated areas of the Field Research Center.

The regulatory requirements for site-specific safety and health plans are found at 29 CFR 1910.120 (b)(4) and include ten specific elements which are designated with the letters A through J. Each of these elements is addressed in this safety and health plan for the Field Research Center. Each element is listed below along with the section number where it is addressed in this safety and health plan.

\section{SAFETY AND HEALTH PLAN ELEMENT}

A) Safety and health risk hazard analysis

B) Employee training assignments and requirements

C) Personal protective equipment requirements

D) Medical surveillance requirements

E) Frequency and types of monitoring required

F) Site control measures

G) Decontamination procedures

H) Emergency response plan

I) Confined space entry procedures

J) Spill containment program
SECTION NO. IN THIS PLAN

4.

$4 ., 5.4$

6.2

4., 5.2

5.3

4., 5.6

5.7

none (no confined space entry)

5.3 


\section{INTRODUCTION AND PROJECT DESCRIPTION}

\subsection{INTRODUCTION}

The Environmental Sciences Division (ESD) at the Oak Ridge National Laboratory (ORNL) has established a Field Research Center (FRC) on the U.S. Department of Energy (DOE) Oak Ridge Reservation (ORR) in Oak Ridge, Tennessee for the DOE Headquarters Office of Biological and Environmental Research. The FRC provides a site for investigators in the Natural and Accelerated Bioremediation Research (NABIR) program to conduct research and obtain samples related to in situ bioremediation. The NABIR program is a ten-year program designed to increase the understanding of fundamental biogeochemical approaches for cleaning up DOE's contaminated legacy waste sites. The FRC is integrated with existing and future laboratory and field research and provides a means of examining the biogeochemical processes that influence bioremediation under controlled, small-scale field conditions.

The FRC lies within the Y-12 Plant area of responsibility on the Oak Ridge Reservation (Fig. 1.1). The Y-12 Plant is located in Bear Creek Valley adjacent to the City of Oak Ridge.

The FRC includes a 243-acre (98-ha), previously disturbed contaminated area to be used for conducting expcriments on a plume of contaminated groundwater. The FRC also includes a 404-acre (163-ha) background area (Fig. 1.1), which provides for comparison studies in an uncontaminated area, and ancillary structures located within a 3.2 -mile $(5.2-\mathrm{km})$ radius of each other on the Oak Ridge Reservation. The FRC contaminated field site includes the commingled groundwater plume found in the shallow unconsolidated sediments ( $<10 \mathrm{~m}$ depth), Nolichucky Shale, and Maynardville Limestone that originated from a combination of the S-3 Waste Disposal Ponds and the Bone Yard/Burn Yard (BY/BY). However, the initial focus of NABIR investigations is on the easily accessible shallow unconsolidated sediments that overlie the Nolichucky Shale. Contaminants in this plume and in the shallow saturated and unsaturated soils include uranium, Tc-99, strontium metal, nitrate, barium, cadmium, volatile organic contaminants (VOCs) and other inorganics and radionuclides believed to be of interest to the NABIR investigators.

The overall procedures for conducting research at the FRC and ensuring regulatory compliance are outlined in the FRC Management Plan. This Site-Specific Health and Safety Plan (HASP) addresses the safety aspects of the spectrum of work activities conducted at the contaminated area of the FRC. The work will be conducted as a series of individual projects by various principal investigators (PIs) from academia, national laboratories, industry, and federal agencies that have been selected to participate in the NABIR Program. FRC research activities are supported by ORNL ESD personnel, and associated ORNL environmental, safety, and health support groups. Activities at the contaminated area (but not the uncontaminated background arca) fall under the scope of Code of Federal Regulations, 29 CFR 1910.120, Hazardous Waste Operations and Emergency Response (HAZWOPER). The purpose of this document is to establish overall site-specific health and safety guidelines to be followed by all personnel conducting work at the FRC, regardless of organizational affiliation. Work will be performed in accordance with requirements, as stipulated, in the ORNL HAZWOPER Program Manual and applicable ORNL, UT-Battelle, and DOE policies and procedures. This HASP serves as an extension of the ORNL HAZWOPER Program Manual, which, combined with the FRC Management Plan, fulfills the requirements of 29 CFR 1910.120. 

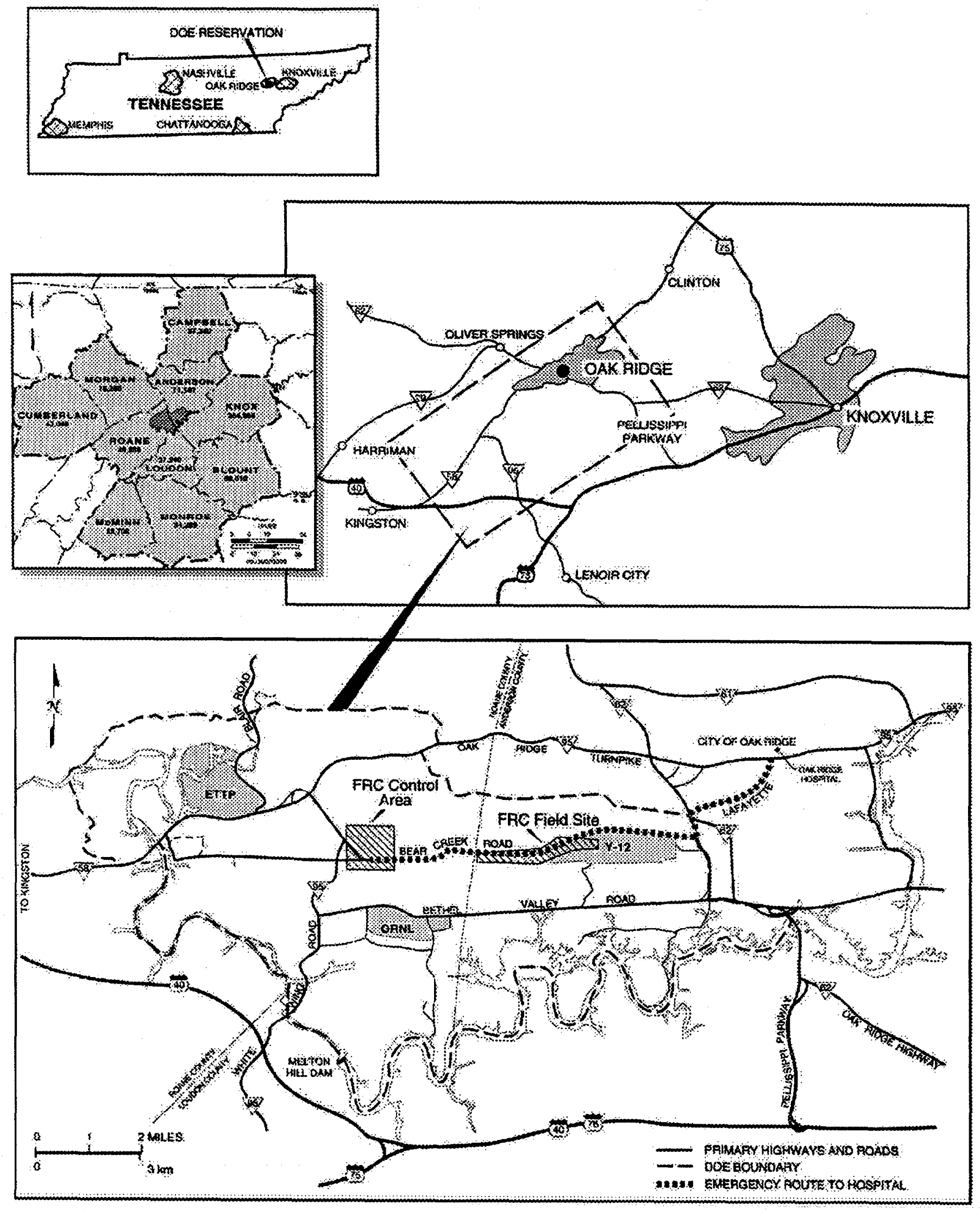

Fig. 1.1 . Location of the FRC in Oak Ridge, Tennessee. 
ORNL strongly emphasizes worker health and safety. Therefore, in addition to the requirements of this HASP, specific environment, safety, health, and quality (ESH\&Q) reviews are conducted for cach individual project proposed for the FRC using the Integrated Safety Management System (ISMS) process. These reviews highlight the specific safety requirements applicable to each project based on its location and project activities. ISMS reviews result in mandatory project-specific ESH\&Q requirements lists, which apply to activities conducted not only at the contaminated area of the FRC (covered by this HASP), but in the uncontaminated background area as well (see FRC Management Plan). Thus, all activities at the FRC are conducted in accordance with ORNL Health and Safety Directives.

The levels of protection and procedures specified in this HASP are based on the best information available from historical data and recent evaluations of the area. Therefore, these recommendations represent the minimum health and safety requirements to be observed by all personnel engaged in work at the FRC. Unforeseeable site conditions, changes in scope of work, or hazardous conditions not previously considered will warrant a reassessment of the protection levels and controls stated. Refer to Sect. 5.1 for requirements pertaining to field modifications and changes to the HASP.

\subsection{DESCRIPTION OF ACTIVITIES CONDUCTED ON THE FRC}

The FRC supports four broad types of activities:

1. Sample acquisition: Source of subsurface samples for use by NABIR investigators.

2. Intrinsic bioremediation analyses: Characterization and analysis of the key subsurface features, processes, and conditions that support intrinsic bioremediation.

3. Accelerated bioremediation research: In situ experimental research on fundamental mechanisms to accelerate bioremediation.

4. Assessment (field-based evaluation) research: In situ evaluation of new methods for characterizing, monitoring and predicting the effectiveness of bioremediation.

Implementing these activities at the FRC involves:

- Installation of groundwater wells and coring

- Drilling (e.g., air rotary, coring, push-probes, hollow-stem auger, sonic)

- Hydrogeologic characterization

- Hydraulic testing (e.g., pumping tests, slug tests, point dilution tests)

- Geophysical testing

- Seismic, neutron, electrical, gamma, sonic, ground penetrating radar, etc.

- Microbiology \& geochemistry

- Groundwater and core samples

- Sampling for other investigators

- Sample preservation in the field (e.g., chemical fixing, freezing with liquid nitrogen) 
- Biogeochemistry

- Groundwater and core samples under controlled atmosphere conditions

- Sample storage and shipment (on \& off site)

- Manipulative tests

- Addition of nutrients (carbon, nitrogen, phosphorous, methane, hydrogen, etc.) for biostimulation

- Addition of chemical stimulants for immobilization of contaminants

- Bacterial Transport Research \& Development - Bioaugmentation

- Enrichments of indigenous, naturally occurring organisms

- Enrichments of non-indigenous naturally occurring organisms 


\section{SITE ORGANIZATION AND COORDINATION}

FRC research activities will be performed by PIs and field project personnel with various affiliations, and by ESD personnel. All work is performed under the direction of the FRC Manager and support staff, all of whom are ESD employees. Appropriate health and safety services including additional monitoring and oversight are provided by the ORNL Office of Safety and Health Protection, and Office of Radiation Protection.

The following section describes the organizational structure of the FRC. Key personnel and their responsibilities are listed. Mr. David Watson is the FRC Manager and Ms. Mary Anna Bogle will serve as the Site Safety and Health Officer (SSHO). Mr. Norman Farrow is the alternate SSHO; Mr. Kirk Hyder will act as the SSHO designee in their absence.

\subsection{SITE SAFETY AND HEALTH OFFICER}

The SSHO advises the FRC Manager on safety and health issues and conducts briefings prior to initiation of site activitics. The SSHO assesses the potential for worker cxposures to hazardous agents, recommends appropriate hazard controls for protection of task site personnel, and will require personnel to obtain immediate medical attention in the event of a work-related injury or illness. The SSHO ensures any necessary monitoring of potential chemical hazards is performed, reviews the effectiveness of monitoring and personal protective equipment, and recommends upgrades or downgrades in protective safety and health measures. The SSHO ensures that appropriate fall protection measures are available and that needed work permits such as Radiological Work Permits (RWPs) are obtained. The SSHO notifies the Office of Radiation Protection when radiological support is required. The SSHO has stop work authority and advises emergency response personnel of an emergency and contacts the Plant Shift Superintendent's Office (PSS) when stop work conditions exist due to suspected health and safety hazard(s). The SSHO authorizes the return to work following resolution of any safety and health hazards or other stop work issues. The SSHO ensures that this HASP is revised and approved if there are changes in site conditions or tasks. The SSHO shall be available for consultation when required and shall be aware of project-related work occurring on-site.

\subsection{FRC MANAGER}

The FRC Manager has primary responsibility for directing and managing all FRC activities, including coordination with Bechtel Jacobs Company (the Environmental Management contractor) and Y-12 support organizations. The FRC Manager ensures that all on-site project personnel meet the required level of training, have reviewed the HASP, and are instructed in safe work practices. The FRC Manager also ensures that a qualified SSHO is designated, maintains a current copy of the HASP, and documents field changes to the HASP in the project logbook. In addition, the FRC Manager and staff perform oversight of field activities, maintain awareness of site operations, and ensure that all project personnel adhere to ES\&H requirements in order to prevent potential accidents from occurring. 
The FRC Manager is responsible for ensuring that the following five core functions of the Integrated Safety Management System are fulfilled appropriately:

- Define the work, roles and responsibilities. Allocate resources to ensure that research goals are balanced with safe work practices.

- Identify and analyze the hazards using the ESH\&Q evaluation, consultation with subject matter experts, material safety data sheet information, Work Smart Standards (WSS), lessons learned by other PIs and staff, and other resources.

- Develop and implement hazard controls tailored to the work being performed.

- Resources include NABIR and FRC staff, ORNL Work Smart Standards, subject matter experts, the Hazardous Materials Inventory System, ESD Chemical Hygiene Plan, Division and project procedures, Training Needs Assessment process, Laboratory Operating Manuals, Laboratory Stewards, ESD ESH\&Q staff, and Lessons Learned and Alerts.

- Examples of actions and tools include optimization of engineering controls and procedural approaches with training, HAZCOM job-specific training, job pre-briefings, compliance-based and project-specific training, ES\&H permits (e.g., RWPs, Lockout/Tagout process), and protective equipment.

- Perform work within controls to ensure the work is done safely:

- Communicate expectations to project staff

- Ensure that the controls identified in the ESH\&Q evaluation and this HASP are carried out

- Ensure opportunity for procedure modification to respond to unanticipated situations

- Stop work if imminent danger exists

- Provide feedback and continuous improvement:

- Solicit feedback from project staff regarding ESH\&Q issues and act on that input

- Communicate concerns to and seek help from supervisors and the ESH\&Q group

- Reallocate resources to address issues that arise

- Ensure safety meetings and site briefings are performed

\subsection{PRINCIPAL INVESTIGATORS AND FIELD PROJECT PERSONNEL}

PIs and field project personnel involved in onsite operations are responsible for understanding the intent of the principles of Integrated Safety Management and are to be knowledgeable of the processes in place to satisfy the intent of Integrated Safety Management.

Define the Scope of Work

- Understand the expectations they are to meet in their particular work assignment

- Understand the responsibilities of the FRC Manager and SSHO

- Provide documentation of training to the FRC Manager 
Identify and Analyze the Hazard

- Notify the SSHO of any special medical conditions (i.e., allergies, diabetes, etc.)

- Actively participate in identification of hazards prior to beginning work

- Ensure that potential work hazards have been evaluated by subject matter experts and are accounted for in all work practices

Develop and Implement Hazard Controls

- Seek the help of the SSHO, ESD's ESH\&Q staff, and other subject matter experts, as appropriate, to analyze the hazards

- Ensure that control strategies are developed and implemented, as appropriate, before work begins

- Ensure safety measures are incorporated into activities (i.e., through HASP addendums or amendments, work aides, or standard operating procedures)

Perform Work Within Controls

- Perform only those tasks that they believe they can do safely

- Meet the responsibilities and safely perform the tasks that are delegated to them

- Take all reasonable precautions to prevent injury to themselves and to their fellow employees; be alert to potentially harmful situations

- Suspend work if unexpected concerns arise and modify plans to address concerns before resuming work

- Comply with the work plan and HASP as well as postings and rules at the project site

Provide Feedback and Continuous Improvement

- Keep the SSHO and FRC Manager informed of any issues, problems, or concerns regarding all aspects of their work

- Notify appropriate facility management personnel or the facility point of contact of any unsafe condition, violation, noncompliance, or environmental threat discovered in a facility

- Report to the SSHO any changes in site conditions that may affect safety and health

- Immediately notify the SSHO of symptoms or signs of exposure potentially related to any chemical, physical, or biological hazards present at the site and immediately report any accidents, injuries, and/or unsafe conditions to the SSHO

- If unsafe conditions develop, task site personnel are authorized and expected to stop work and notify the SSHO and FRC Manager of the unsafe condition.

\subsection{RADIATION PROTECTION}

The ORNL Office of Radiation Protection will be responsible for oversight and approval of personal protection requirements related to radiation protection. A Radiation Protection representative will be contacted for all radiological concerns at the site. Relevant directives include ORNL Radiation Protection Program, RP-110, ORNL Radiological Control Policy and the ORNL Radiological Protection Procedures, and 10 CFR 835, Occupational Radiation Protection. 


\section{INTEGRATED SAFETY MANAGEMENT SYSTEM}

The ISMS process systematically integrates safety into management and work practices at all levels so missions are accomplished while protecting the public, the worker, and the environment. Direct involvement of workers during the development and implementation of safety management systems is essential for success. DOE requires that the principles of ISMS be implemented for all ORNL activities. Therefore, all FRC personnel are expected to incorporate the following basic ISMS core functions during all work activities:

- Defining the scope of work;

- Identifying and analyzing hazards associated with the work;

- Developing and implementing hazard controls;

- Performing work activities within these controls; and

- Providing feedback on the adequacy of the controls to continue improving safety management. 


\section{TASK SPECIFIC HAZARD EVALUATION AND CONTROLS}

The purpose of this FRC hazard evaluation is to identify and assess potential hazards that personnel might encounter and to prescribe methods of hazard control. Data tables A-1, A-2, and A-3 in Attachment A give the results of chemical and radiological contaminant analyses in soils, groundwater, and surface water at representative locations within the FRC contaminated areas. Material Safety Data Sheets (MSDS) for chemicals that are likely to be handled when conducting field work are included in Attachment B.

A description of sampling procedures and the activities to be conducted at the FRC is described below, and is also provided in greater detail in the NABIR FRC Site Characterization Plan and FRC Management Plan.

\subsection{FIELD SAMPLING AND ANALYSIS OF GROUNDWATER}

Task Description: Procedures for field sampling and analysis of groundwater are described in the FRC Site Characterization Plan. Groundwater will generally be sampled with a peristaltic pump. Slow purge techniques will be used in order to reduce the disturbance caused by removal of large volumes of water from the system. Field parameters will be monitored until stable groundwater chemistry (e.g. specific conductance, $\mathrm{pH}, \mathrm{Eh}$, temperature, dissolved oxygen) readings are obtained on the YSI Sonde. The YSI Sonde will be fitted with a flow-through cell to reduce exposure to the atmosphere. The purge water will be collected in 55-gallon drums and removed by the environmental management contractor, Bechtel Jacobs Company. It may also be possible to discharge water back to the subsurface if approved by the regulators.

Samples will be handled and transported according to regulatory requirements and procedures outlined in the FRC Storage and Data Management Plan, and the FRC Management Plan. Samples will be preserved and stored as required by the analytical protocols (e.g. cooled, preservative added) and FRC health-physicists will check for exterior radioactivity. Storage on site may occur for short periods of time in ice chests containing "blue ice" but will be quickly transferred to refrigerator storage at the laboratory at the appropriate temperatures. All storage of contaminated samples will follow procedures and regulations relevant to radioactive materials. Special handling procedures may be required to maintain anoxic sterile conditions for some microbial samples collected.

Equipment and Materials: Sampling equipment includes sampling tube (remains in the monitoring wells); peristaltic pump; a YSI multi-probe flow cell (for measuring water ficld parameters); filters and sample containers (for collecting samples); $\mathrm{HACH}$ meter and test kits and reagents for analyzing sulfur, ferrous iron, nitrite/nitrate, dissolved oxygen, and carbonate (see Appendix B for reagent MSDSs). Some samples will be preserved with a few drops of nitric, hydrochloric or sulfuric acid or formaldehyde. Calibration standards including $\mathrm{pH}$ and conductivity are also used. Stand-alone probes will be used for soil gross radioactive screening, downhole uranium analysis, $\mathrm{pH}, \mathrm{Eh}$, and groundwater specific conductance measurements. Compressed gas cylinders filled with nitrogen, argon, or other inert gases may be used to flush the void space in sample containers of oxygen prior to and/or after sampling. 


\section{Task Hazards and Controls:}

- Chemical and Radiological Hazards

- Groundwater Contact: Based on previously obtained sample data, the risk of chemical or radiological exposure from short-term exposure to groundwater and surface water samples is minimal (Tables A-1, A-2, and A-3 give the results of chemical and radiological contaminant analyses in soils, groundwater, and surface water at representative locations within the FRC contaminated areas). However, direct contact with contaminated materials should be avoided, therefore, disposable latex or nitrile gloves and safety glasses will be worn when conducting groundwater sampling to prevent eye and skin contact.

- Reagent Contact: Corrosive or oxidizing reagents pose a contact hazard. To prevent eye and skin contact when corrosive or oxidizing reagents are used disposable latex or nitrile gloves and safety glasses will be worn.

- Physical Hazards

- Compressed gas cylinders: Rules and practices for handling compressed gas cylinders can be found at http://www-internall ornl.gov/oshp/nec suff/gasinfo.htm.

- Tripping/Falling: Precautions should be taken to avoid trip, slip, and fall accidents when climbing irregular or slippery surfaces. Before changing location visually survey the area for slippery surfaces and tripping hazards.

- Heat/Cold Stress: Wear clothing appropriate for environmental and weather conditions. Temperature extremes may be a hazard for consideration depending on the timing of the activity. Refer to Section 5.5 for discussion of recognition of symptoms and controls.

\section{- Explosion Hazards}

- Gas cylinders: Pressurized gas cylinders will be transported and handled in accordance with applicable Department of Transportation and ORNL guidance and regulations. Care will be taken to secure the cylinders upright during transport to ensure they are not damaged. Cylinders will also be secured at the site so they will not tip over during the injection process. Rules and practices for handling compressed gas cylinders can be found at http://wwwinternall.ornl.gov/oshp/nec suff/gasinfo.htm.

- Biological/Vector Hazards

- Ticks/Snakes/Pathogens: Be cautious of snakes, and vector carriers such as ticks. Check clothing and skin for ticks after walking in brush. Wash hands before eating and drinking.

- Personal Protective Equipment Required to Address General Site Hazards

- Level of Protection: D

- Protective Clothing: DOE-issued work clothes or disposable tyvek 
- Head Gear: Safety glasses

- Gloves: Latex or nitrile (when conducting groundwater sampling or handling corrosive or oxidizing reagents)

- Footwear: Sturdy work shoes

- Monitoring Requirements

- Radiological Monitoring: Although no radiological exposure is expected, the need for radiological monitoring will be reviewed by the Radiological Technicians prior to the start of work.

\subsection{TRACER TESTS OR MICROBIAL MANIPULATION AND TRANSPORT STUDIES}

Task Description: A description of tracer testing procedures is provided in the NABIR FRC Site Characterization Plan. Microbial manipulative tests may involve injection of nutrients (carbon sources, nitrogen, phosphorous, methane, hydrogen, etc.) into wells for biostimulation or the addition of chemical stimulants for immobilization of contaminants. Bacterial transport research may involve enrichments of indigenous, naturally occurring organisms or the addition of non-indigenous naturally occurring organisms in groundwater. The tracer or microbial stimulant may be injected/pumped directly into the injection well, or in some instances an injection system will consist of a double straddle packer design that will be used to isolate the screened interval in the injection well. A computational datalogger may be used to automatically deliver small increments of a concentrated tracer stock solution through a solenoid valve in order to maintain a constant diluted injection concentration within the packed interval. The fluid in the injection interval will be continuously mixed by re-circulating groundwater through a peristaltic pump and flow-through temperature-compensated conductivity cell. Copper refrigeration tubing may be used for the entire circulation system except for a $15-\mathrm{cm}$ long piece of silicon tubing needed in the pump head. Copper tubing will eliminate the loss of dissolved gas tracers (e.g., $\mathrm{He}, \mathrm{Ne}$, $\mathrm{SF}_{6}, \mathrm{Kr}$ ) that may be co-injected into the same wells. The gas injection and sampling system utilizes molecular diffusion through gas-permeable tubing. Gas is continuously pumped through a coil of Teflon tubing installed in the screened section of the well. The gas diffuses quickly into the surrounding groundwater, coming to a steady-state concentration based on the in situ temperature and pressure conditions, the solubility constant, and the average groundwater flux through the well screen.

Gas samples will be obtained from monitoring wells using passive-diffusive samplers consisting of two parts-a length of gas-permeable silicon tubing, and a copper sample tube that is sealed on one end and valved on the other. Dissolved gas diffuses through the silicon tubing and comes to equilibrium with the surrounding groundwater. A portion of the gas is trapped in the copper tube, which is open to the silicon tubing by way of a valve while the sampler is suspended in the well. When the sampler is removed from the well, the silicon tubing is immediately disconnected, closing the valve and sealing in the gas sample. Alternatively, water samples may be obtained using methods similar to those used for sampling some VOCs. In that case, headspace samples would be analyzed for the gas tracers. Anionic tracer and microbial samples are generally collected as described in Sect. 4.1 for groundwater field sampling, although special procedures may be required to maintain anoxic sterile conditions for the microbial samples. 
Equipment and Materials: Equipment includes sampling tube (remains in the monitoring wells); peristaltic pump; a YSI multi-probe flow cell (for measuring water field parameters); filters and sample containers (for collecting samples). Non-toxic tracers that may be used include: bromide, fluorescent dyes; ice-nucleating bacteria (INA), PFBA, PIPES, He, Ne, $\mathrm{SF}_{6}, \mathrm{Kr}$ and other noble gases. Gas cylinders will be used to transport and store gases. Nutrients may include carbon sources (e.g., lactate), nitrogen, phosphorous, methane, and hydrogen.

\section{Task Hazards and Controls:}

\section{- Chemical and Radiological Hazards}

- Groundwater Contact: Based on previously obtained sample data, the risk of chemical or radiological exposure from short-term exposure to groundwater and surface water samples is minimal (Tables A-1, A-2, and A-3 give the results of chemical and radiological contaminant analyses in soils, groundwater, and surface water at representative locations within the FRC contaminated areas). However, direct contact with contaminated materials should be avoided, thercfore, disposable latex or nitrile gloves and safety glasses will be worn when conducting groundwater sampling to prevent eye and skin contact.

- Reagent Contact: Corrosive or oxidizing reagents pose a contact hazard. To prevent eye and skin contact when corrosive or oxidizing reagents are used disposable latex or nitrile gloves and safety glasses will be worn.

\section{- Physical Hazards}

- Compressed gas cylinders: Rules and practices for handling compressed gas cylinders can be found at http://www-internall ornl.gov/oshp/nec_suff//gasinfo.htm.

- Tripping/Falling: Precautions should be taken to avoid trip, slip, and fall accidents when climbing irregular or slippery surfaces. Before changing location, visually survey the area for slippery surfaces and tripping hazards.

- Heat/Cold Stress: Wear clothing appropriate for environmental and weather conditions. Temperature extremes may be a hazard for consideration depending on the timing of the activity. Refer to Sect. $\mathbf{5 . 5}$ for discussion of recognition of symptoms and controls.

- Lifting: Use your legs to lift heavy objects; avoid awkward positions and twisting of the body and ask for assistance with awkward or heavy loads.

\section{- Explosion Hazards}

- Gas cylinders: Pressurized gas cylinders will be transported and handled in accordance with applicable DOT and ORNL guidance and regulations. Care will be taken to secure the cylinders upright during transport to ensure they are not damaged. Cylinders will also be secured at the site so they will not tip over during the injection process. Rules and practices for handling gas cylinders can be found at http://www-internall.oml.gov/oshp/nec_suff//gasinfo.htm. 


\section{- Biological/Vector Hazards}

- Ticks/Snakes/Pathogens: Be cautious of snakes, and vector carriers such as ticks. Check clothing and skin for ticks after walking in brush. Wash hands before eating and drinking.

\section{- Personal Protective Equipment Required to Address General Site Hazards}

- Level of Protection:

- Protective Clothing: DOE-issued work clothes or disposable tyvek

- Head Gear: Safety glasses

- Gloves: Latex or nitrile (when conducting groundwater sampling or handling corrosive or oxidizing reagents)

- Footwear: Sturdy work shoes

- Monitoring Requirements

- Radiological Monitoring: Although no radiological exposure is expected, the need for radiological monitoring will be reviewed by the Radiological Technicians prior to the start of work.

\subsection{WATER LEVEL MEASUREMENTS}

Task Description: Manual water levels are taken by lowering an electronic water level sounder down the well. As the sounder is brought out of the well the tip of the sounder that has been submerged is rinsed with distilled water to rinse off the groundwater. The rinse water is allowed to drip back down into the well. Sampling tubes at some locations have to be removed prior to taking a water level measurement. The top of the well casing will be surveyed for location and elevation.

Water level measurements taken with data loggers and transducers will be downloaded with a portable computer via a cable that runs out of the well. Contact with subsurface contaminants is not likely to occur when downloading data. Transducers will be rinsed when moved from well to well.

Equipment and Materials: Equipment includes water level sounder, transducers, data loggers, computers, and survey equipment

\section{Task Hazards and Controls:}

\section{- Chemical and Radiological Hazards}

- Groundwater Contact: Based on previously obtained sample data, the risk of chemical or radiological exposure from short-term exposure to groundwater and surface water samples is minimal (Tables A-1, A-2, and A-3 give the results of chemical and radiological contaminant analyses in soils, groundwater, and surface water at representative locations within the FRC contaminated areas). However, direct contact with contaminated materials should be avoided; 
therefore, disposable latex or nitrile gloves and safety glasses will be worn when conducting groundwater monitoring and during the handling of sample tubes and downhole transducers to prevent eye and skin contact.

- Downhole equipment: Rinse downhole equipment with distilled water as it is brought out of the well. Scanning of downhole equipment for possible radiological contamination will be required if the equipment is to be moved offsite but not when moving equipment between wells.

\section{- Physical Hazards}

- Tripping/Falling: Precautions should be taken to avoid trip, slip, and fall accidents when climbing irregular or slippery surfaces. Before changing location visually survey the area for slippery surfaces and tripping hazards.

- Heat/Cold Stress: Wear clothing appropriate for environmental and weather conditions. Temperature extremes may be a hazard for consideration depending on the timing of the activity. Refer to Section 5.5 for discussion of recognition of symptoms and controls.

- Biological/Vector Hazards

- Ticks/Snakes/Pathogens: Be cautious of snakes, and vector carriers such as ticks. Check clothing and skin for ticks after walking in brush. Wash hands before eating and drinking.

- Personal Protective Equipment Required to Address General Site Hazards

- Level of Protection: D

- Protective Clothing: DOE-issued work clothes or disposable tyvek

- Head Gear: Safety glasses

- Gloves: Latex or nitrile (when conducting groundwater sampling or handling corrosive or oxidizing reagents)

- Footwear: Sturdy work shoes

- Monitoring Requirements

- Radiological Monitoring: Although no radiological exposure is expected, the need for radiological monitoring will be reviewed by the Radiological Technicians prior to the start of work

\subsection{HYDRAULIC TESTING (E.G., PUMPING TESTS, SLUG TESTS, POINT DILLUTION TESTS AND FLOW METERS SUCH AS THE COLLOIDAL BORESCOPE)}

Task Description: Pumping tests are conducted by pumping groundwater from a well at a constant rate of discharge. Water levels in the pumped well and nearby well are monitored with a water level 
sounder or data logger and transducer. The groundwater will be discharged to storage containers like 55 -gallon drums or in some cases to the ground if regulatory approval is obtained.

Slug tests are conducted by placing a solid slug into a well and displacing a known amount of water. The slug is quickly removed and water levels are taken (with a transducer and data logger or manually with a water level sounder) to monitor groundwater recovery in the well.

Point dilution tests in uncased wells will be conducted by using a dual packer apparatus to isolate a small section of the well. For drivepoint wells targeting designated depths within the formation, the method will utilize a single packer apparatus to isolate the screened interval since the bottom of the well is already sealed. Groundwater in the test interval will be slowly replaced with deionized water and the specific conductance and pressure-head monitored continuously.

Flow meter (e.g., colloidal borescope or electromagnetic borehole flowmeter) measurements are taken by lowering the flowmeter into a well and using above-ground equipment (i.e., computers) to monitor the rate and direction of flow. The equipment is rinsed off with distilled water as it is removed from the well.

Equipment and Materials: Equipment includes peristaltic pumps, well packers and compressor to inflate the packers, water level sounder, transducers, data loggers and computers.

\section{Task Hazards and Controls:}

\section{- Chemical and Radiological Hazards}

- Groundwater Contact: Based on previously obtained sample data, the risk of chemical or radiological exposure from short-term exposure to groundwater and surface water samples is minimal (Tables A-1, A-2, and A-3 give the results of chemical and radiological contaminant analyses in soils, groundwater, and surface water at representative locations within the FRC contaminated areas). However, direct contact with contaminated materials should be avoided; therefore, disposable latex or nitrile gloves and safety glasses will be worn when conducting groundwater sampling to prevent eye and skin contact.

\section{- Physical Hazards}

- Tripping/Falling: Precautions should be taken to avoid trip, slip, and fall accidents when climbing irregular or slippery surfaces. Before changing location visually survey the area for slippery surfaces and tripping hazards.

- Heat/Cold Stress: Wear clothing appropriate for environmental and weather conditions. Temperature extremes may be a hazard for consideration depending on the timing of the activity. Refer to Sect. 5.5 for recognition of symptoms and controls.

- Lifting: Use your legs to lift heavy objects, avoid awkward positions and twisting of the body and ask for assistance with awkward or heavy loads. 


\section{- Biological/Vector Hazards}

- Ticks/Snakes/Pathogens: Be cautious of snakes, and vector carriers such as ticks. Check clothing and skin for ticks after walking in brush. Wash hands before eating and drinking.

\section{- Personal Protective Equipment Required to Address General Site Hazards}

- Level of Protection: D

- Protective Clothing: DOE-issued work clothes or disposable tyvek

- Head Gear: Safety glasses

- Gloves: Latex or nitrile (when conducting groundwater sampling or handling corrosive or oxidizing reagents)

- Footwear: Sturdy work shoes

\section{- Monitoring Requirements}

Radiological Monitoring: Although no radiological exposure is expected, the need for radiological monitoring will be reviewed by the Radiological Technicians prior to the start of work

\subsection{CORING AND WELL INSTALLATION}

Task Description: Pushprobes are installed by using a drill rig either hydraulically pushing or hammering steel drive pipe into the ground (please refer to the FRC Site Characterization Plan for a more complete description of coring and well installation). Sections of piping are added (threaded attachment) until the desired depth is reached. A small diameter well can then be constructed inside the hollow pipe as it is withdrawn from the ground or in the uncased hole after the drive pipe is removed completely. The drive point is either left in the ground or withdrawn through the hollow drive pipe. Soil cores can be taken during drilling operations by driving split-spoon or other types of samplers. The core samples will be scanned and processed at the surface. Special procedures and use of compressed inert gas such as nitrogen or argon may be required to maintain anoxic sterile conditions for some core samples that will have microbial analyses conducted on them. Sampling equipment and drive pipe is cleaned and decontaminated by pressure washing. Hollow-stem augers and other drilling methods may also be used to install groundwater monitoring wells. These methods produce drill cuttings that need to be contained and managed according to procedures outlined in the FRC Management Plan and site specific research plan prepared for the project.

Equipment and Materials: Equipment includes drill rigs and associated equipment and support vehicles such as air compressors, pressure washers, generators, drill rod, containment boxes equipped with HEPA filters, and well construction materials. A glove bag and compressed gas cylinders may be used to process cores that require microbial analyses. 


\section{Task Hazards and Controls:}

\section{- Chemical and Radiological Hazards}

- Groundwater Contact: Based on previously obtained sample data, the risk of chemical or radiological exposure from short-term exposure to groundwater and surface water samples is minimal (Tables A-1, A-2, and A-3 give the results of chemical and radiological contaminant analyses in soils, groundwater, and surface water at representative locations within the FRC contaminated areas). However, direct contact with contaminated materials should be avoided, therefore, disposable latex or nitrile gloves and safety glasses will be worn when conducting groundwater sampling to prevent eye and skin contact.

- Soil/cuttings Contact: Workers could be exposed to contaminated soil remaining on the piping as it is raised out of the ground. This hazard will be minimized by scanning the drive pipe as it is raised out of the hole. Prior to removal from the site, all drill pipe, drill cuttings, and any core samples collected will be scanned for radionuclide contamination. An exclusion area will be set up around the drill rig to prevent entry by personnel that are not trained or wearing proper protection. A minimum of two people are required for this operation. An RWP will be required if drilling is to be performed in areas contaminated with radionuclides, and containment boxes equipped with HEPA filters will be used as specified by the RWP.

\section{- Physical Hazards}

- Compressed gas cylinders: Rules and practices for handling compressed gas cylinders can be found at http://www-internall ornl gov/oshp/nec_suff/gasinfo.htm.

- Tripping/Falling: Precautions should be taken to avoid trip, slip, and fall accidents when climbing irregular or slippery surfaces. Before changing location visually survey the area for slippery surfaces and tripping hazards.

- Heat/Cold Stress: Wear clothing appropriate for environmental and weather conditions. Temperature extremes may be a hazard for consideration depending on the timing of the activity. Refer to Sect. 5.5 for discussion of recognition of symptoms and controls.

- Abrasions, Scrapes and Sprains: Always use appropriate care when using tools and mechanical equipment. Maintain awareness of body and limb location and think ahead to probable body and object path before applying force to tools. Wear protective clothing as listed below.

- Lifting: Use your legs to lift heavy objects, avoid awkward positions and twisting of the body and ask for assistance with awkward or heavy loads.

- Mechanical Hazard: Working with drill rigs can result in injuries from equipment dislodging and striking unsuspecting personnel, and from impacts due to flying objects or overturning vehicles. Therefore, follow these precautions:

- Drill rig brakes, hydraulic lines, light signals, fire extinguishers, fluid levels, steering, tires, horns, and other safety devices will be inspected routinely throughout the project.

- Drill rig cabs will be kept free of all nonessential items and all loose items will be secured.

- Drill rigs will be provided with necessary safety equipment, including seat belts. 
- Parking brakes will be set before shutting off any heavy equipment or vehicle.

- Electrical Hazard: Of special concern to drilling operations is the possibility for conducting electricity through the drilling tower through either inadvertent contact with underground or overhead power lines, or by lightning strikes. In addition, some of the equipment used is operated by electricity. Unless safe work practices are observed, serious injury or death can result. Therefore, observe the following precautions:

- Treat all electrical wires and circuits as "live" unless certain they are not.

- Always maintain a firm work base to prevent a loss of balance and potential fall onto energized busses or parts (which should be covered with a good electrical insulator such as a rubber blanket).

- All tools should have insulated handles, be electrically grounded, or double insulated.

- Do not drill within $10 \mathrm{ft}$ of an overhead power line that is $\leq 50 \mathrm{kV}$ (or within $50 \mathrm{ft}$ for $>50 \mathrm{kV}$ ) unless power to the line is first turned off for the duration of the drilling.

- Ground fault circuit interrupters will be used for electrical extension cords in use between a fixed electrical system (permanent outlet) and a tool.

- Prior to drilling have site representatives delineate location of underground power lines and other utilities (accomplished through penetration permits as part of ISMS process).

- Do not drill within $25 \mathrm{ft}$ of any known underground power line.

- Maintain a watch for electrical storms. If electrical activity appears to be imminent, cease drilling operations and evacuate the area around the drill rig. If time permits do not leave auger or drill string in the borehole.

- Noise: Unprotected exposure of site workers to noise from drilling activities can result in noise induced hearing loss. Hearing protection must be worn where noise levels are greater than 85 dBA. The SSHO will ensure that either ear muffs or disposable foam earplugs are made available to all personnel and are used by the personnel in the immediate vicinity of the drill rig.

\section{- Explosion Hazards}

- Gas cylinders: Pressurized gas cylinders will be transported and handled in accordance with applicable DOT and ORNL guidance and regulations. Care will be taken to secure the cylinders upright during transport to ensure they are not damaged. Cylinders will also be secured at the site so they will not tip over during the injection process. Rules and practices for handling gas cylinders can be found at http://www-internall.ornl.gov/oshp/nec suff/gasinfo.htm.

\section{- Biological/Vector Hazards}

- Ticks/Snakes/Pathogens: Be cautious of snakes, and vector carriers such as ticks. Check clothing and skin for ticks after walking in brush. Wash hands before eating and drinking.

\section{- Personal Protective Equipment Required to Address General Site Hazards}

- Level of Protection: D

- Protective Clothing: DOE-issued work clothes or disposable tyvek

- Head Gear 
- Hard hat required for drill rig operations; not required for steam cleaning and washing

- Safety glasses or goggles required during drilling and dccon opcrations

- Ear muffs or disposable foam earplugs required in the vicinity of drill rig

- Gloves: Leather work gloves over nitrile of latex gloves during drilling or decon operations

- Footwear: Steel-toed work shoes

\section{- Monitoring Requirements -}

- Radionuclide Contamination: Prior to being removed from the site, all drill pipe, drill cuttings and any core samples collected will be scanned for radionuclide contamination. An exclusion area will be set up around the drill rig to prevent entry of people that are not trained or wearing the proper protection. A minimum of two people is required for this operation. An RWP will be required if drilling in areas contaminated with radionuclides.

- Radiological Monitoring: Although no radiological exposure is expected, the need for radiological monitoring will be reviewed by the Radiological Technicians prior to the start of work

- Air Quality: Air monitoring with an organic vapor analyzer or other suitable instrument will be required if in areas where soils or groundwater heavily contaminated with VOCs may be brought to the surface. However, high VOC concentrations are not expected to be encountered at the FRC. 


\section{OTHER HEALTH AND SAFETY PLAN ELEMENTS}

\subsection{REVISIONS/ MODIFICATIONS TO THE HASP}

The following actions will warrant revision and approval of this plan by the appropriate health and safety disciplines:

- Change in tasks (or previously unidentified tasks) that could impact employee health and safety

- Changes in hazards (unknown or not previously addressed) which require a significant change in, or addition to, respiratory protection (as defined in exemptions to the plan modifications), physical/barrier protection features, or other engineering controls

- Occurrences as defined by DOE Order 232.1A

\subsubsection{Modifications allowed}

Radiological Conditions. Under the direction of the field health physics technician, the level of personal protective equipment (PPE) may be upgraded including use of air-purifying respirators, for radiological issues. These changes must be documented in the field logbook. The specific change and reason or evidence for the change must be documented in the field logbook. Any upgrades beyond airpurifying respirators will require additional review/approval from the health and safety disciplines and revision of this plan. The RWP shall be continuously updated to reflect the radiological hazards of the work environment.

Non-Radiological Conditions. The SSHO may upgrade PPE for non-radiological issues and contaminants. These changes must be documented in the field logbook. The change and reason or evidence for the change must also be documented in the field logbook. For upgrades to include respiratory protection (including air-purifying and supplied air) for previously unidentified nonradiological issues or contaminants such as VOCs, the appropriate health and safety disciplines must be contacted. The SSHO will approve and document changes in PPE in the field logbook. Upgrades to include respiratory protection will require the SSHO to ensure workers have 40 Hour HAZWOPER Training and to assess any additional medical surveillance requirements.

\subsection{MONITORING}

Both historical site data and current well monitoring data indicate that chemical and radiological exposure or radiological contamination of site personnel is not a significant concern within the scope of this project. Therefore, other than for coring and well installation (see Section 4.5) no additional monitoring is required unless there is reason to suspect, based on information identified in the project specific ESH\&Q requirements reviews conducted through the ISMS process, that chemical or radiological contamination will be encountered during field activities.

Instruments used by ORNL Industrial Hygiene representatives will be calibrated and maintained in accordance with Industrial Hygiene Standard Operating Procedures. Instruments used by the Office of Radiation Protection are calibrated and source checked in accordance with established Health Physics procedures. Site monitoring requirements may change based on site conditions. All changes must be documented in the site logbook. 


\subsection{SITE AND SPILL CONTROL}

Site access is available from public roads through the area and therefore will not be controlled to the general site. Based on the anticipated levels of contamination, formal barricaded work zones will not be established unless new monitoring data indicate the need for such barriers. An exclusion zone may be required for drilling operations if required to reduce the accidental spread of hazardous substances from contaminated areas to clean areas. The Health Physics representative will determine the need for radiological postings. The SSHO will determine, as needed, the locations of the support zone, contamination reduction zone, and the exclusion zone. Personnel accessing the zones must meet access requirements as stated in this plan.

Only milliliter quantities of reagents which could pose hazards will be used at a time on this project. Required controls when using such reagents are latex or nitrile gloves and safety glasses. Emergency eye wash shall be present and operational at the site. Best management practices will be employed to minimize and control spillage. MSDSs for many of the reagents are included in Appendix B. MSDSs for additional chemicals can be accessed through the ORNL web server at http:/hmweb.ctd.ornl.gov/msds/msds home.htm. In the event of eye contact with corrosive or oxidizing reagents, an emergency eye wash shall be used. For eye or skin contact immediately flush affected areas with water for 15 minutes.

\subsection{PERSONAL PROTECTIVE EQUIPMENT}

Level $\mathrm{D}$ protection is normally used when the potential for personnel contamination is low, as is the case with this project. Level D protection will include DOE-furnished clothing or disposable tyvek. Details and special requirements have been covered in the hazard control sections of the specific tasks in Sect. 4 above. Unexpected new hazards will require a reassessment of the specified PPE.

\subsection{TEMPERATURE EXTREMES AND SITE CHARACTERISTICS}

The effect of temperature extremes on personnel is a primary hazard associated with the activities conducted at the FRC. Symptoms and controls related to temperature extremes are considered in detail in this section.

Field activities conducted during the summer or winter pose a hazard because of temperature extremes. Since the project site is located in a relatively open area, workers shall dress appropriately for environmental conditions, wearing clothing that provides reasonable protection against winter cold and summer sun. Although extreme physical exertion will not be likely within the scope of this project, during hot weather workers are encouraged to be aware of their own symptoms of heat stress (headaches, dizziness, increased heart rate), to drink plenty of water, and to take breaks as needed. Heat stress symptoms, remedies, and monitoring are discussed in Sect. 5.5.1. Cold exposure effects are discussed in Sect. 5.5.2.

Workers are also encouraged to apply insect repellant and/or sunscreen as needed prior to field activities. Workers should exercise caution by visually inspecting their immediate area of activity for presence of poisonous/harmful plant, insect, and animal species as well as any hazard resulting from previous human activity. 


\subsubsection{Effects and Prevention of Heat Stress}

If the body's physiological processes fail to maintain a normal body temperature because of excessive heat, a number of physical reactions can occur. They can range from mild symptoms such as fatigue, irritability, anxiety, and decreased concentration, dexterity, or movement, to death.

Heat-related health concerns can include the following:

- Heat rash: Caused by continuous exposure to heat and humid air and aggravated by chafing clothes. Decreases ability to tolerate heat and is a nuisance.

- Heat cramps: Caused by profuse perspiration combined with inadequate fluid intake and chemical replacement, particularly salts. Signs include muscle spasm and pain in the extremities and abdomen.

- Heat exhaustion: Caused by increased stress on various organs to meet increased demands to cool the body. Signs include shortness of breath; increased pulse rate (120-200 beats per minute); pale, cool, moist skin; profuse sweating; dizziness; and lassitude.

- Heat stroke: Is the most severe form of heat stress. Body must be cooled immediately to prevent severe injury and/or death. Signs include red, hot, dry skin; no perspiration; nausea; dizziness and confusion; strong, rapid pulse; and possibly coma. Medical help must be obtained immediately.

Medical attention must be obtained for the more serious symptoms of heat stress. One or more of the following methods is recommended to help reduce the potential for heat stress:

1. Provide plenty of liquids. To replace body fluids (water and electrolytes) lost due to sweating, use a 0.1 percent saltwater solution, more heavily salted foods, or commercial mixes. The commercial mixes may be preferable for those employees on a low-sodium diet.

2. Provide cooling devices to aid natural body ventilation. These devices, however, add weight, and their use should be balanced against worker efficiency.

3. Wear long cotton underwear, which acts as a wick to help absorb moisture and protect the skin from direct contact with heat-absorbing protective clothing.

4. Install mobile showers and/or hose-down facilities to reduce body temperature and cool protective clothing.

5. In extremely hot weather, conduct non-emergency response operations in the early morning or evening.

6. Ensure that adequate shelter is available to protect personnel against sun, heat, or other adverse weather conditions that decrease physical efficiency and increase the probability of accidents.

7. In hot weather, rotate workers wearing protective clothing.

8. Maintain good hygiene frequently changing clothing and showering daily. Clothing should be permitted to dry during rest periods. Workers who notice skin problems should immediately consult medical personnel. 


\subsubsection{Cold Exposure}

Persons working outdoors in temperatures at or below freezing may suffer from cold exposure. During prolonged outdoor periods with inadequate clothing for protection, the effects of cold exposure may occur even at temperatures well above freezing. Cold exposure may cause severe injury due to freezing of exposed body surfaces (frostbite), or profound generalized cooling (hypothermia), possibly resulting in death. Areas of the body which have high surface area-to-volume ratios such as fingers, toes, and ears are the most susceptible to frostbite.

Local injury resulting from cold is included in the generic term frostbite. There are several degrees of damage. Frostbite of the extremities can be categorize into:

- Frost nip or incident frostbite: characterized by sudden blanching or whitening of skin.

- Superficial frostbite: skin has a waxy or white appearance and is firm to the touch, but tissue beneath is resilient.

- Deep frostbite: tissues are cold, pale, and solid; extremely serious injury.

Systemic hypothermia, or lowering of the core body temperature, is caused by exposure to freezing or rapidly dropping temperatures. Symptoms are usually exhibited in five stages: 1) shivering and loss of coordination; 2) apathy, listlessness, sleepiness, and (sometimes) rapid cooling of the body to less than $\left.95^{\circ} \mathrm{F}\left(35^{\circ} \mathrm{C}\right) ; 3\right)$ unconsciousness, glassy stare, slow pulse, and slow respiratory rate; 4) freezing the extremities; and 5) death.

\subsection{DECONTAMINATION}

Decontamination of personnel shall be conducted only in the unexpected event that contamination is detected. Decontamination efforts shall be carried out in accordance with Radiological Surveillance Section Standard Operating Procedure 02-540-01, Handling Radiologically Contaminated Personnel. At a minimum, personnel who have conducted work at the site will wash their hands prior to eating or drinking. Health Physics personnel shall supervise, assist, and document incidents involving personnel contamination.

If contamination is detected on the drilling equipment, ESD decontamination equipment is available for use such as a steam cleaner, pressure washer, water reservoir tank, and a wastewater transfer system and receiving tank.

Radiological decontamination efforts shall be conducted with the guidance of Health Physics personnel. A Radiation Protection Specialist may be reached at telephone (865) 576-1408. 


\subsection{EMERGENCY PREPAREDNESS/RESPONSE}

Personnel will not be in residence at the FRC and therefore, a formal emergency response plan is not appropriate. The site is in close proximity to several occupied buildings at the Y-12 Plant. In the event of an area evacuation, personnel will muster at Y-12 Assembly Point 21. The Y-12 Plant emergency response organization will be contacted for response to all emergencies at the project site. Emergency medical treatment and first aid is immediately available at the Y-12 Plant. All emergency services can be reached by dialing 911 from any plant telephone. Access to phones and/or radios will be provided to onsite personnel. The Plant Shift Supervisor will coordinate all emergency response operations.

Should evacuation from the site become necessary, the evacuation route to the hospital in the City of Oak Ridge is shown in Fig. 1.1. Emergency telephone numbers are given below. For a complete listing of individuals and organizations important to operation of the FRC, refer to the FRC Management Plan.

\section{Emergency Telephone Numbers}

Y-12 Plant Shift Supervisor

Y-12 Medical Services

ORNL Laboratory Shift Superintendent

ORNL Medical Services

FRC Manager

Radiation Protection Specialist

ESH\&Q Assurance Specialist
(865) 574-7172

or 911 from any plant telephone

(865) 574-1583 (Building 9706-2)

or 911 from any plant telephone

(865) 574-6606

(865) 574-7537 (Building 4500N, Rm K-125)

(865) 241-4749

(865) 576-1408

(865) 873-4829 pager

(865) 574-7837 


\section{TRAINING/MEDICAL REQUIREMENTS}

\subsection{SITE-SPECIFIC HAZARD COMMUNICATION AND ACCESS BRIEFING}

Since different training requirements may be needed based on the nature of different tasks to be performed, specific training requirements (e.g., RAD Worker II, Respirator) will be identified in the project specific ESH\&Q requirements lists. However, generally applicable training requirements are presented here.

Visitors not entering any exclusion zone or contamination reduction zone who have very limited potential for exposure to contaminants require:

- Site-specific hazard communication and access briefing

All project personnel performing hands-on work that could potentially expose them to hazardous substances, safety, or health hazards will meet the following training requirements:

- General Employee Training and General Employee Radiological Training (GET/GERT)

- 40 hour HAZWOPER (SARAJOSHA) training, or equivalent (Note: for certain types of low risk work, 24 hour training is acceptable)

- Current HAZWOPER 8-hour Annual Refresher (as applicable)

- Site-specific hazard communication and access briefing

In addition, the Site Safety and Health Officer requires:

- 8-hour HAZWOPER Supervisor training

Personnel involved in service or maintenance work on energized equipment require:

- Lockout/Tagout training

Prior to beginning work at the project site, all personnel will review this Health and Safety Plan and sign the training acknowledgment form (Appendix $\mathrm{C}$ ). The site-specific hazard communication and access briefing is documented in the project logbook. If site conditions change, or other hazards are detected, the training and access requirements will be revised accordingly. For example, if radiological contamination is detected, only those who have satisfactorily completed Radiological Worker Training II may perform hands-on work in the contaminated area. 


\subsection{MEDICAL SURVEILLANCE}

A medical surveillance program will be conducted in accordance with the requirements of 29 CFR 1910.120 for:

- All employees who are or may be exposed to hazardous substances or health hazards at or above the established permissible exposure limits or, if there is no permissible exposure limit, above the published exposure levels for these substances, without regard to the use of respirators, for 30 days or more a year

- All employees who wear a respirator for 30 days or more a year or as required by 29 CFR 1910.134

- All employees who are injured, become ill or develop signs or symptoms due to possible overexposure involving hazardous substances or health hazards from an emergency response or hazardous waste operation

- Members of HAZMAT teams

All ORNL employees receive periodic medical examinations. Because of the low potential for exposure to hazardous agents, it is not expected that additional medical surveillance will be required for ORNL personnel at the FRC. Non-ORNL personnel will be required to acknowledge coverage by a medical surveillance program sufficient to satisfy the requirements of 29 CFR 1910.120 (Appendix C). 
Appendix A

DATA TABLES 
Table A-1. Results of chemical analysis of wastes, soils, groundwater, and surface water at the S-3 Ponds Site FA

\begin{tabular}{|c|c|c|c|c|c|c|c|c|c|c|c|c|c|}
\hline \multirow[b]{2}{*}{ 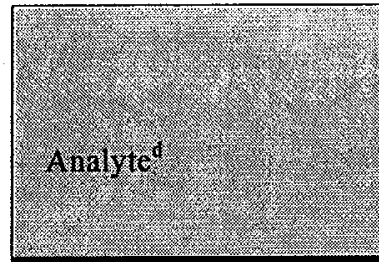 } & \multirow[b]{2}{*}{$\begin{array}{c}\text { Wastes } \\
\text { S-3 } \\
\text { sludges }\end{array}$} & \multicolumn{4}{|c|}{ Soils" } & \multicolumn{4}{|c|}{ Groundwater $^{b}$} & \multicolumn{4}{|c|}{ Surface Water } \\
\hline & & $\begin{array}{l}\text { Resuits } \\
\text { detection } \\
\text { limit }\end{array}$ & $\begin{array}{l}\text { Meximum } \\
\text { Matect } \\
\text { intect }\end{array}$ & $\begin{array}{l}0 \\
\text { Average } \\
\text { resulit } \\
\end{array}$ & SRC? & $\begin{array}{l}\text { Freq. of } \\
\text { wells w. } \\
\text { detects }\end{array}$ & $\underset{\text { detect }}{\operatorname{Max}}$ & $\begin{array}{l}\text { Mean of } \\
\text { the } \\
\text { medians }\end{array}$ & SRC? & $\begin{array}{l}\text { Frequof } \\
\text { detect }\end{array}$ & $\begin{array}{r}\text { Avgt } \\
\text { resuit } \\
\text { Bek } 12.4 \\
6\end{array}$ & $\begin{array}{l}\text { A yg. } \\
\text { resuit } \\
\text { NI-1 } \\
\end{array}$ & SR \\
\hline \multicolumn{14}{|c|}{ Inorganics (water - $\mathrm{mg} / \mathrm{L}$, soils - $\mathrm{mg} / \mathrm{kg}$ ) } \\
\hline Juminum & 22134 & 401.40 & 29000 & 15600 & & $23 / 23$ & 800 & 81.05 & $Y$ & 20182 & 0.506 & 0.56 & \\
\hline ntimeny & & 21.2 & 0.34 & 0.3 & & $0 / 20$ & & .025 & & & & & \\
\hline rsenic: & 1.12 & $40 / 40$ & 20.5 & 3.5 & & $1 / 9$ & 0.009 & .003 & & 2 & 2 & $2=$ & \\
\hline arium: & 133.5 & 40140 & 712 & 145 & 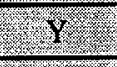 & $24 / 24$ & 380 & 19.137 & $\mathrm{Y}$ & 77178 & 0,092 & 0,554 & \\
\hline emMiliur & 2.74 & 36140 & .1 .8 & 10 & & $11 / 22$ & 0.11 & 0.0119 & $Y$ & 3178 & 0.0000 & 0.0002 & \\
\hline aron & 27.64 & $\mathrm{NA}$ & 2 & $\sqrt{25}$ & $2=$ & $24 / 24$ & 4.40 & .29 & $Y$ & 78178 & 0.086 & 0.073 & \\
\hline admiun & 4.9 & $2 / 40$ & -2 & 0.2 & & $10 / 25$ & 4 & 0.174 & $Y$ & 31.4 & 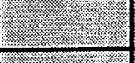 & 0.015 & \\
\hline Calcium & 42215.6 & 39139 & 318000 & 26700 & & $26 / 26$ & 10,000 & $1,665.6$ & & 78178 & 164.5 & 2283 & \\
\hline Chromium: & 133.2 & 40240 & 69.6 & 308 & 2 & $13 / 25$ & 0.31 & 011 & $Y$ & 2 & 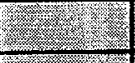 & 5 & \\
\hline sobalt & .47 & 40140 & 311 & 16.6 & 5 & $11 / 23$ & 2.3 & 0.268 & $\mathrm{Y}$ & $5 / 78$ & 0.001 & 0.007 & \\
\hline Copper & 92.45 & 38140 & 50.8 & 22.3 & $Y$ & $20 / 21$ & 3.1 & 0.278 & $\bar{Y}$ & 4178 & 0.005 & 0.002 & \\
\hline Cyanide & 5.95 & 41.40 & 7.8 & 0.6 & $y$ & $0 /$ & & & & 6172 & 0.001 & 2. & \\
\hline Iron & 3647 & 40140 & 48300 & 25600 & 8 & $23 / 23$ & 28 & 3.59 & & 46178 & 0.743 & 0.233 & \\
\hline Lead & 35.79 & $4 0 \longdiv { 4 0 }$ & 82.3 & 15.5 & $y$ & $21 / 26$ & 0.66 & 0.0106 & $Y$ & 114 & 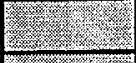 & 0.003 & \\
\hline Linium & 75.68 & 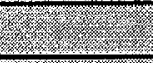 & 2 & $8=$ & $\sqrt{25}$ & $7 / 7$ & 3.5 & 0.88 & $\mathrm{Y}$ & $33 / 72$ & 0.015 & 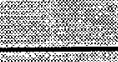 & \\
\hline Magnesiun & 1941.32 & $40 \% 40$ & 60400 & 6930 & $\sqrt{2}$ & $26 / 26$ & 2,500 & 263.3 & & 78178 & 23.4 & 298 & \\
\hline Wanganese & 99.67 & 40140 & 2000 & 707 & 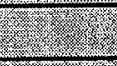 & $24 / 24$ & 220 & 24.162 & $Y$ & 78178 & 0.046 & 3.0 & \\
\hline Mercury & 2.28 & $26 / 39$ & 82.9 & 7.8 & $Y$ & $15 / 26$ & 0.1100 & 0.0042 & $\mathrm{Y}$ & 181.72 & 0.0000 & & \\
\hline Molybdenum & 15.2 & 1140 & 3.7 & 0.5 & & $3 / 19$ & 0.023 & 0.006 & & 2 & & & \\
\hline Nickel 2020 & 550.32 & $40 / 40$ & 128 & $29: 6$ & $y$ & $22 / 24$ & 20 & 2.07 & $Y$ & 5178 & 0.005 & 0.068 & \\
\hline Niobium & 20.87 & 8.2 & 2.20 & 2 & & $4 / 5$ & 0.10 & 0.027 & $Y$ & & & 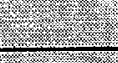 & \\
\hline Phosphorous & 952.9 & 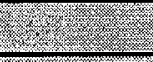 & 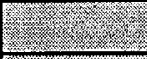 & 20 & & & & & & 41172 & 0.058 & 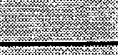 & \\
\hline otassium & 1625.6 & 40140 & 3990 & 2120 & $\sqrt{2}$ & $26 / 26$ & 200 & 30.95 & & 78178 & 5.126 & 4.5 & \\
\hline elenium & 0.77 & 21.40 & 0.66 & 0.31 & 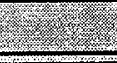 & $3 / 10$ & 0.014 & 0.003 & & 112 & 0.001 & & \\
\hline
\end{tabular}




\begin{tabular}{|c|c|c|c|c|c|c|c|c|c|c|c|c|c|}
\hline \multirow{2}{*}{ 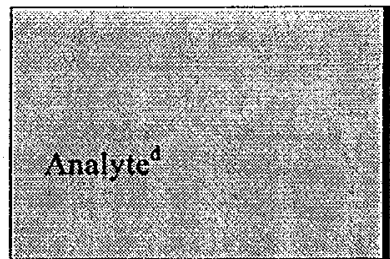 } & \multirow{2}{*}{$\begin{array}{c}\text { Wastes } \\
\text { S-3 } \\
\text { sludges }\end{array}$} & \multicolumn{4}{|c|}{ Soils ${ }^{2}$} & \multicolumn{4}{|c|}{ Groundwater $^{\mathbf{b}}$} & \multicolumn{4}{|c|}{ Surface Water: } \\
\hline & & $\begin{array}{l}\text { Results } \\
\text { detection } \\
\text { limit }\end{array}$ & Maximum & $\begin{array}{l}\text { Avetage } \\
\text { result }\end{array}$ & SRe? & $\begin{array}{l}\text { Freq. of } \\
\text { wells w. } \\
\text { detects }\end{array}$ & $\begin{array}{l}\text { Max } \\
\text { detect }\end{array}$ & $\begin{array}{l}\text { Mean of } \\
\text { the } \\
\text { medians }\end{array}$ & SRC? & $\begin{array}{l}\text { Freq: of } \\
\text { defect }\end{array}$ & $\begin{array}{l}\text { Avg: } \\
\text { resuit } \\
\text { BCK } 12.4 \\
6\end{array}$ & $\begin{array}{l}\text { Avg: } \\
\text { resuit: } \\
\text { NT-1 } \\
\end{array}$ & \\
\hline Silicon & & 2 & $\sqrt{2}$ & 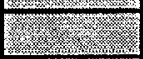 & $1=$ & $24 / 24$ & 39 & 9.99 & & 2 & $=$ & 5 & \\
\hline Silver & 1.87 & 26140 & 2.4 .3 & 1.8 & U. & $0 / 6$ & 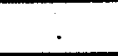 & & & & 2.0 & & \\
\hline Sodium : : & 2722.2 & 32140 & 313 & 117 & 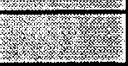 & $26 / 26$ & 3,200 & 357.6 & & 78178 & 57.04 & 29.5 & \\
\hline Strontium & 35.49 & 25 & 2 & 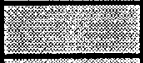 & 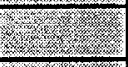 & $22 / 22$ & $\overline{340}$ & 17.93 & $\mathrm{Y}$ & 781.78 & 0.42 & 0.692 & \\
\hline Thalliun & 0.33 & 2140 & 0.75 & 0.22 & $\sqrt{18}$ & $1 / 8$ & 0.01 & 0.005 & & 2172 & 0.001 & 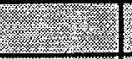 & \\
\hline Thoriun & 195.1 & 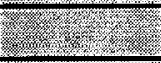 & 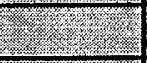 & 2 & 8 & $1 / 20$ & 0.28 & 0.109 & & 2172 & 0.006 & $8=$ & \\
\hline $\min$ & 1.57 & 3140 & .7 .7 & 1.7 & & $3 / 6$ & 0.018 & 0.01 & & 2.2 & 2.0 & 8 & \\
\hline Titarium & 398.6 & 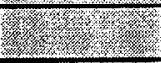 & 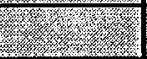 & 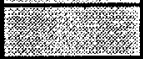 & 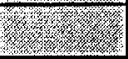 & $7 / 7$ & 0.85 & 0.237 & $\mathrm{Y}$ & 3172 & 0.009 & & \\
\hline Total iraniun (livorometric) & 991.71 & 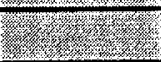 & 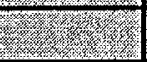 & 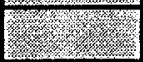 & 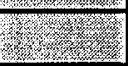 & $22 / 26$ & 44 & 2.90 & $\mathrm{Y}$ & 78178 & 0.621 & 0.075 & \\
\hline Vanadium & 7.1 & 401.40 & 91.9 & 24.4 & $y=$ & $5 / 20$ & 0.036 & 0.004 & $\mathrm{Y}$ & 1172 & 0.003 & es: & \\
\hline inc & 58.27 & 40140 & 163. & 50.1 & $y$ & $24 / 24$ & 20 & 0.497 & $\mathrm{Y}$ & 30178 & 0.013 & 0.014 & \\
\hline Zrrconium & 478.4 & 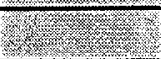 & 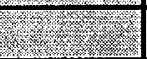 & 2 & 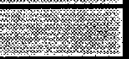 & $3 / 4$ & 0.051 & 0.018 & & 11.71 & 0.002 & 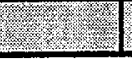 & \\
\hline \multicolumn{14}{|c|}{ Common Anions (water - mg/L, soils - mg/kg) } \\
\hline Ammonia nitrogen & & 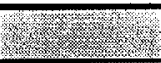 & 2 & 20. & & $2 / 4$ & 4.8 & 1.19 & & 5 & & 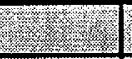 & \\
\hline Chloride: & & 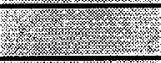 & 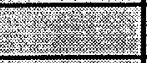 & 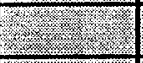 & & $26 / 26$ & 1,341 & 106.2 & $\mathrm{Y}$ & $6 / 6$ & 5 & 27.4 & \\
\hline Fluoride & & $2=0$ & 8 & 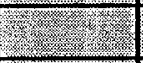 & & $23 / 25$ & 110 & 1.32 & $\bar{Y}$ & 6.6 & & 1.0 & \\
\hline Jeldahi nitrogen & 460 & 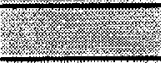 & 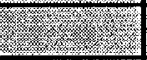 & 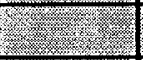 & 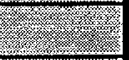 & $4 / 4$ & 1.8 & 0.73 & & 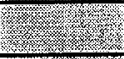 & 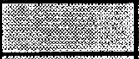 & $2.2=$ & \\
\hline Nitrate $-\mathrm{N}=3$ & 1130 & $\sqrt{2.2}$ & 2 & 2. & & $19 / 26$ & 13,400 & 1,659 . & $\mathrm{Y}$ & 77177 & 38.83 & 166.6 & $y$ \\
\hline Nitrite & & 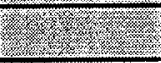 & 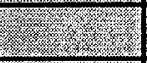 & 2 & 2 & $1 / 4$ & 6.7 & 20.2 & & 8 & & 2. & \\
\hline Sulfate & 220 & 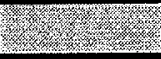 & $2=1$ & 8 & 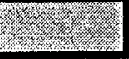 & $23 / 23$ & 2,204 & 204.7 & $Y$ & $6 / 6$ & & 46.8 & \\
\hline \multicolumn{14}{|c|}{ Organics (water - $\mathrm{mg} / \mathrm{L}$, soils - $\mathrm{mg} / \mathrm{kg}$ ) } \\
\hline Endrin aldehyde & & -1113 & .5 .4$. & 2.3 & $\mathrm{Y}=$ & & & & & 2.0 & 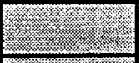 & & \\
\hline Methoxychlor & & $1 / 13$ & 5.5 .9 & 9.8 & $\mathrm{Y}$ & & & & & 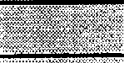 & $=2$ & 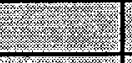 & (2. \\
\hline PCB. 1248 & 5666.8 & 4439 & 3300 & 108 & $\mathrm{Y}^{2}$ & & & & & 20 & 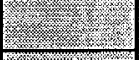 & & 20 \\
\hline $\mathrm{PCB}-1254$ & & 15139 & .370 & 59 & $\mathrm{Y}$ & & & & & 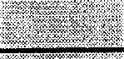 & 2.2 & & 10 \\
\hline $\mathrm{RCB}-1260$ & & 6139 & 280 & 38.2 & $Y$ & & & & & 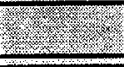 & & 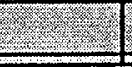 & \\
\hline
\end{tabular}




\begin{tabular}{|c|c|c|c|c|c|c|c|c|c|c|c|c|c|}
\hline & Wastes & 1.: & Soils & 2 & & & Groum & water ${ }^{b}$ & & 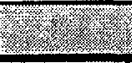 & Surface & Vater: & 3 \\
\hline Aralyife ${ }^{d}$ & $\begin{array}{c}\text { S-3 } \\
\text { sludges }\end{array}$ & $\begin{array}{l}\text { Rescins } \\
\text { detection } \\
\text { limit }\end{array}$ & $\begin{array}{l}\text { Naximum } \\
\text { deteot }\end{array}$ & $\begin{array}{l}\text { Average: } \\
\text { result }\end{array}$ & $\operatorname{sic} ?$ & $\begin{array}{l}\text { Freq. of } \\
\text { wells w. } \\
\text { detects }\end{array}$ & $\begin{array}{l}\text { Max } \\
\text { detect }\end{array}$ & $\begin{array}{l}\text { Mean of } \\
\text { the } \\
\text { medians }\end{array}$ & SRC? & $\begin{array}{l}\text { Freq of } \\
\text { detect }\end{array}$ & 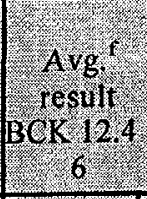 & $\begin{array}{l}\text { Avg? } \\
\text { result } \\
\text { NI-1 }\end{array}$ & SRC. \\
\hline 191-Trekiloroeliare & & 203 & 26xis? & $\sqrt{2.32}$ & & $9 / 27$ & 28 & 3.8 & $Y$ & & & & \\
\hline 1.12-2richloroething & 75.37 & 8 & 80 & 2 & & $1 / 26$ & 1 & 2.5 & & 30 & 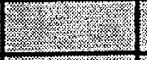 & & \\
\hline 1, 1,2-Tetrachiloroethane & 383.5 & 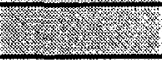 & 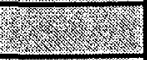 & 20 & & & & & & & & & \\
\hline 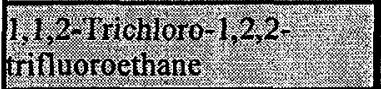 & 4.35 & & & & & & & & & & & & \\
\hline FitePichloroetherie & & 8 & & $\sqrt{20}$ & 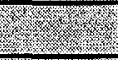 & $3 / 26$ & 5 & 2.5 & & & & & \\
\hline 1,2-Dichloroethane & & 20 & 20 & 3 & 3 & $1 / 26$ & 4 & 2.5 & & & 80.4 & & \\
\hline 1,2-Dichioroethene & & 10 & $\sqrt{3.2}$ & 2 & 8 & $3 / 25$ & 100 & 5.0 & $Y$ & 9.3 & & 20 & \\
\hline 2-Butoxy ellianol & 2289.6 & 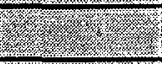 & . & 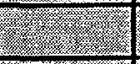 & 8 & & & & & 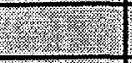 & & 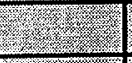 & \\
\hline 20-Butoxsethoxy) Ethang & 1908.0 & 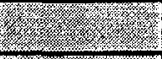 & 1. & 3 & & & & & & & & 93 & \\
\hline 2,4-Dinitrophenol. & & 8 & 2.5 & 20 & & $1 / 3$ & 77 & 20.8 & $\mathrm{Y}$ & & 20 & 8 & \\
\hline 2-Butanone & & $\sqrt{2+3}$ & 20 & 2 & & $9 / 26$ & 32 & 5.0 & $Y$ & 12 & 20 & -2 & \\
\hline 2-Chloroethylvinyl ether & 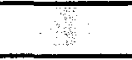 & 2 & 2880 & 20 & 2 & $1 / 10$ & 4 & 5 & & 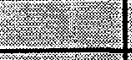 & $1=$ & 30 & \\
\hline 2 Hexanone & & $21 \cdot 39$ & 17 & 6.5 & YS: & $3 / 26$ & 4 & 5 & & 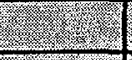 & & & \\
\hline 2-Hexanol & 295.7 & 2 & 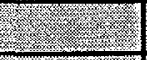 & 3 & 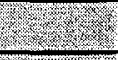 & & & & & $=$ & & & \\
\hline 2-Nitrophenol so & & 8 & 20 & 2.2 & & $1 / 3$ & 71 & 20.3 & $\mathrm{Y}$ & 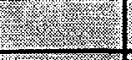 & 2.0 & 20 & \\
\hline $\begin{array}{l}\text { 3-(1, Dinethylethy)- } \\
\text { phenol }\end{array}$ & 6038.8 & & & & & & & & & & & & \\
\hline 4-Metiyl-2-pentanone & & 5140 & 28 & 6.4 & 1 & $1 / 27$ & 97 & 8.4 & & 2 & 2 & 0 & \\
\hline 4-Nitrophenol. & & 2 & 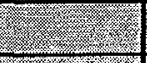 & 2.2 & & $1 / 3$ & 120 & 30.3 & $Y$ & 2 & 2 & 8 & \\
\hline Acetone & 22.95 & $1 7 \longdiv { 3 2 }$ & 4200 & 275 & $y$ & $19 / 27$ & 6,000 & 51.8 & $\mathrm{Y}$ & 115 & & 8.5 & Y. \\
\hline Benzene & & 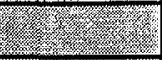 & 20 & 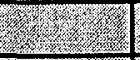 & 2 & $4 / 26$ & 3 & 2.5 & $\mathrm{Y}$ & 2 & 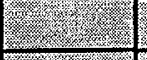 & 8 & \\
\hline Benzenernethanol. & & $x^{2}$ & 8 & 20 & 3 & $1 / 3$ & 2 & 4.2 & & 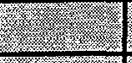 & 2 & 3 & 2 \\
\hline Benzid acia & & (2) & 3 & $\sqrt{2}+2$ & 2 & $2 / 3$ & 58 & 25.0 & Y & 2 & 3 & 30 & \\
\hline Bis(2-ethylhexyl)ohithalate & & 203 & 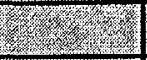 & 20 & 2 & $2 / 3$ & 32 & 6.3 & $\mathrm{Y}$ & 5 & 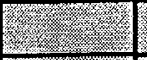 & 28 & \\
\hline Bromodichloromethane & & (2) & 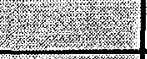 & 2.2. & 1. & 2/ 26 & 3 & 2.5 & & & 10 & 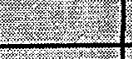 & \\
\hline Bromoform & & 20 & $2=2$ & 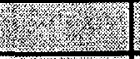 & 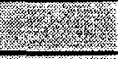 & $1 / 26$ & 2 & 2.5 & & 2 & 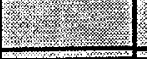 & 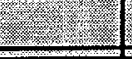 & \\
\hline
\end{tabular}




\begin{tabular}{|c|c|c|c|c|c|c|c|c|c|c|c|c|c|c|c|c|c|c|c|c|c|c|c|c|c|c|c|}
\hline & $\frac{9}{3}$. & & & & & & & & & & & & & & & & $\gamma$ & 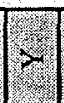 & & & & & & 7 & & 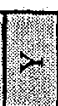 & \\
\hline 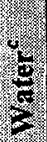 & 85 & & & & & & & & & ? & & L & & & & & & ? & & & & & & 8 & & वे: & \\
\hline . & $\begin{array}{l}46 \\
7\end{array}$ & & . & (3) & 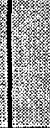 & 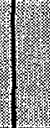 & & & & & & & . & & & & $\frac{5}{5}$ & 2 & & 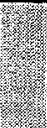 & 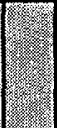 & & & $\frac{3}{2}$ & & $\frac{\square}{3}$ & \\
\hline & $\begin{array}{l}0.83 \\
\frac{8}{9} \frac{8}{8}\end{array}$ & & & 8 & & & . & & & & & & 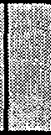 & & . & & $\frac{1}{5}$ & . & & 8 & . & 8 & & $\frac{\infty}{2}$ & . & $\frac{\infty}{\infty}$ & . \\
\hline \multirow{4}{*}{ 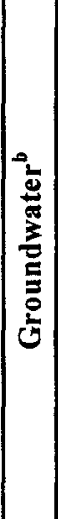 } & $\ddot{\tilde{s}}$ & & & & & $\lambda$ & & & & & & & $>$ & & $>$ & & $>1$ & $>1$ & 2 & & $>$ & & & $\lambda$ & $\mid>$ & $>$ & $>$ \\
\hline & 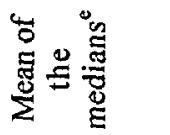 & $m$ & $\begin{array}{l}n \\
i\end{array}$ & $\sim$ & $\begin{array}{l}n \\
i\end{array}$ & $\therefore$ & & $n$ & $\stackrel{n}{i}$ & & $\stackrel{T}{*}$ & $m$ & $\begin{array}{l}n \\
i\end{array}$ & $\begin{array}{l}n \\
r\end{array}$ & $\stackrel{?}{=}$ & $\ddot{m}$ & $\begin{array}{c}0 \\
\dot{8} \\
0\end{array}$ & $\mid \begin{array}{c}2 \\
\hat{\sigma} \\
i\end{array}$ & $\stackrel{r}{n}$ & $\stackrel{\infty}{i}$ & $\hat{i}$ & & n & $\frac{5}{6}$ & $\stackrel{?}{r}$ & $\left|\begin{array}{l}\infty \\
0 \\
0 \\
0 \\
i \\
\sim\end{array}\right|$ & m \\
\hline & 递䔏 & - & in & 10 & $m$ & 8 & 6 & $\theta$ & - & & $N$ & $m$ & $\stackrel{0}{0}$ & $\nabla$ & $\begin{array}{l}8 \\
\text { in }\end{array}$ & - & $\left|\begin{array}{l}\tilde{\delta} \\
0 \\
0\end{array}\right|$ & $\left|\begin{array}{l}8 \\
8 \\
0 \\
0\end{array}\right|$ & $m$ & $N$ & 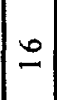 & & - & \begin{tabular}{l|l}
00 \\
0 \\
0
\end{tabular} & $\mid \begin{array}{l}\infty \\
i \\
i\end{array}$ & $\begin{array}{l}8 \\
8 \\
n \\
n\end{array}$ & $a$ \\
\hline & 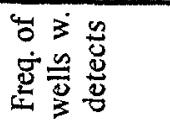 & $\frac{m}{n}$ & in & $\stackrel{8}{2}$ & $\stackrel{\text { i }}{\text { i }}$ & $\frac{7}{3}$ & i & is & $\stackrel{\substack{N \\
ٍ}}{=}$ & & $\stackrel{m}{=}$ & $\bar{m}$ & $\frac{i}{i n}$ & $\frac{\substack{N \\
m}}{m}$ & $\begin{array}{l}\bar{N} \\
\bar{a}\end{array}$ & $\stackrel{m}{=}$ & $\bar{\lambda}$ & $\left|\begin{array}{l}\bar{\lambda} \\
\bar{a}\end{array}\right|$ & $\stackrel{\aleph}{\triangleq}$ & $\frac{m}{\lambda}$ & $\frac{N}{0}$ & & i & 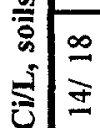 & \pm & $\stackrel{\infty}{\Xi}$ & $\frac{\nabla}{\partial}$ \\
\hline \multirow{4}{*}{$\frac{2}{6}$} & & & & 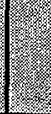 & & 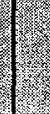 & 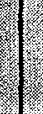 & & & & & & 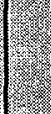 & & . & & & 3 & $>$ & & & & & 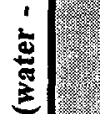 & 3 & & \\
\hline & 80 & & & & 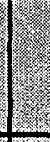 & & & & & & & & & & 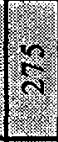 & & 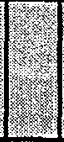 & (2) & 2 & & ? & & & $\begin{array}{l}2 \\
0\end{array}$ & $\sqrt{8}$ & $\frac{6}{6}$ & \\
\hline & $\frac{3}{3} \frac{3}{2}$ & & & & & & & & & & & & & & $\frac{8}{4}$ & & & त. & בิ & & & & & $\frac{2}{2}$ & $\frac{\mathrm{a}}{2}$ & $\therefore$ & . \\
\hline & $\frac{x}{3} \frac{9}{8} \frac{9}{8}=$ & s & & Ses & & 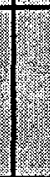 & & & 8 & & & ? & & & $y^{2}$ & & 8 & $\frac{9}{6}$ & $\frac{8}{2}$ & 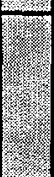 & . & & 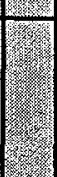 & $\frac{8}{2}$ & $\sqrt{9}$ & $\frac{8}{7}$ & 8 \\
\hline & 总总 & & & & & & & & & 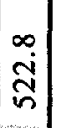 & & & & & g & & $\begin{array}{c}\infty \\
\infty \\
\vdots \\
m \\
n\end{array}$ & 울. & $\stackrel{\tilde{y}}{0}$ & & $\begin{array}{c}\infty \\
\stackrel{\sim}{0}\end{array}$ & $\begin{array}{l}\infty \\
\vdots \\
0 \\
0\end{array}$ & & तें & 0 & $\begin{array}{l}y \\
y \\
\dot{g} \\
\vdots\end{array}$ & $\stackrel{\infty}{+}$ \\
\hline & $\sqrt{\frac{3}{3}}$ & $\frac{\frac{3}{g}}{\frac{3}{3}} \frac{5}{3}$ & $\left(\begin{array}{l}\frac{3}{3} \\
\frac{\pi}{5} \\
\frac{5}{6} \\
\frac{6}{5}\end{array}\right.$ & $\left(\frac{8}{\frac{8}{5}}\right.$ & $\mid \begin{array}{l}0 \\
\frac{8}{5} \\
\frac{3}{8} \\
\frac{5}{5}\end{array}$ & $\sqrt{\frac{5}{E}}$ & 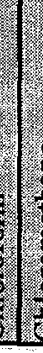 & $\frac{8}{8}$ & 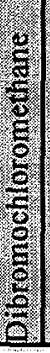 & 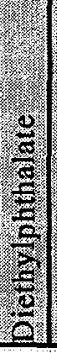 & $\begin{array}{l}\frac{3}{\pi} \\
\frac{3}{5} \\
\frac{5}{2} \\
\frac{3}{6} \\
\frac{5}{0}\end{array}$ & 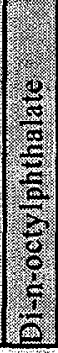 & $\begin{array}{l}\frac{3}{5} \\
\frac{5}{5} \\
\frac{5}{2} \\
\frac{2}{5} \\
\frac{5}{6}\end{array}$ & 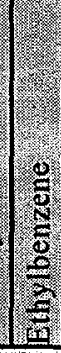 & $\mid \begin{array}{l}\frac{8}{6} \\
\frac{6}{3} \\
\frac{9}{5} \\
\frac{2}{3} \\
\frac{2}{3}\end{array}$ & (2) & $\left|\begin{array}{c}3 \\
5 \\
7 \\
6 \\
5 \\
5 \\
5 \\
\frac{5}{9} \\
2\end{array}\right|$ & 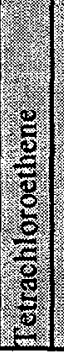 & (1) & 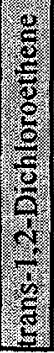 & (2) & 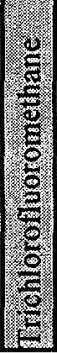 & $\begin{array}{l} \\
\frac{8}{8} \\
\frac{8}{8} \\
\frac{3}{3}\end{array}$ & $\frac{3}{\frac{3}{3}}$ & $\mid \frac{\frac{\pi}{9}}{\frac{5}{5}}$ & $\left(\begin{array}{l}0 \\
\frac{3}{8} \\
\frac{3}{2} \\
\frac{2}{2}\end{array}\right.$ & $\sqrt{3}$ \\
\hline
\end{tabular}




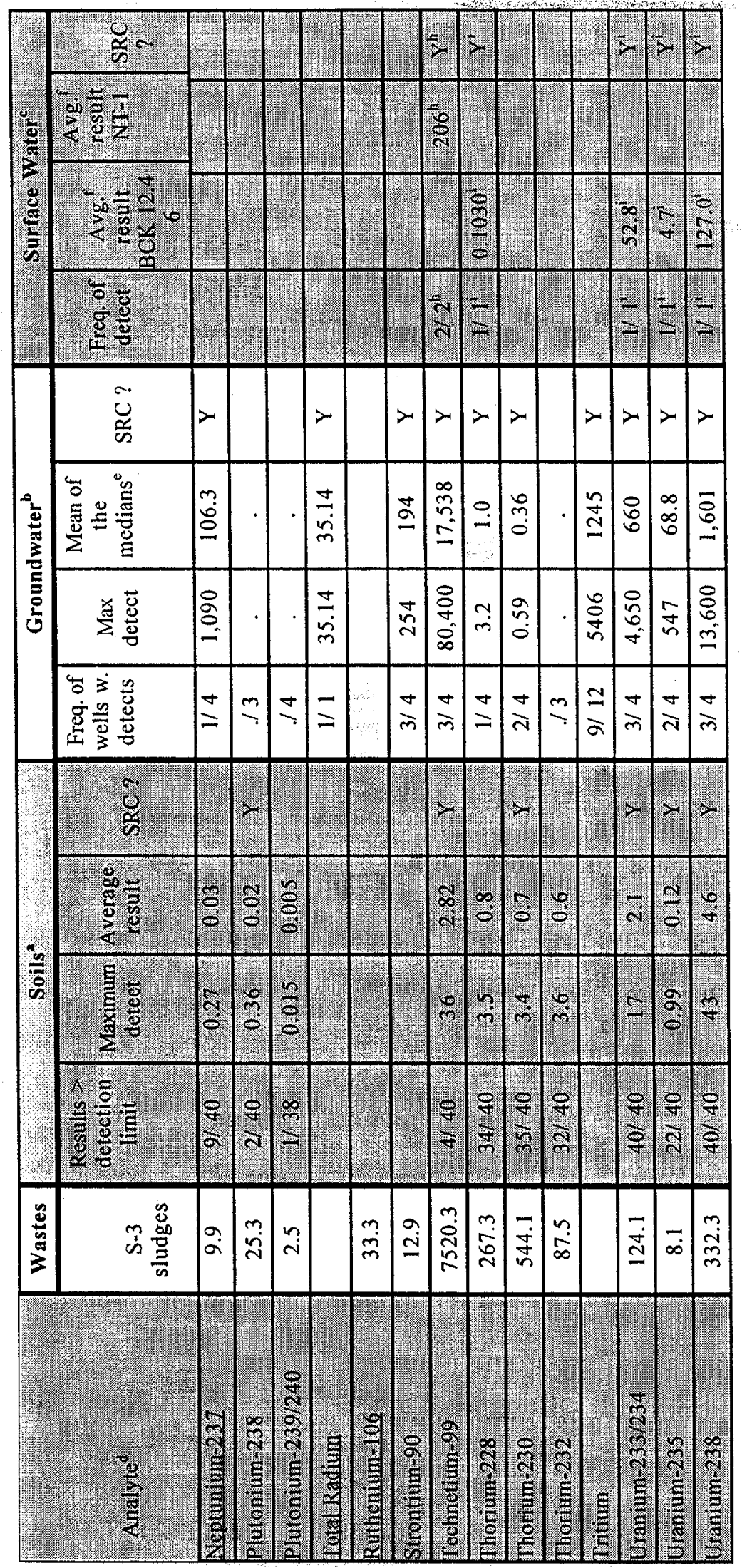

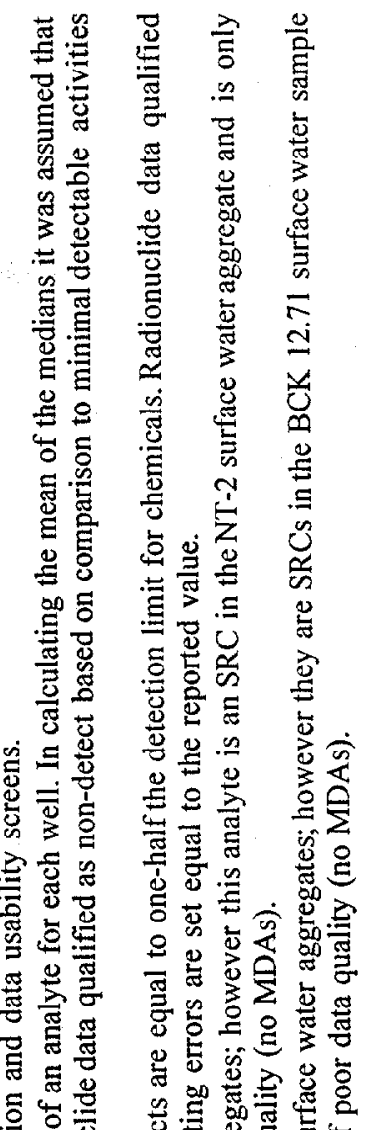

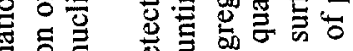

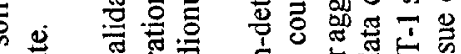

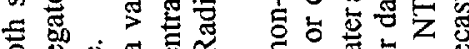

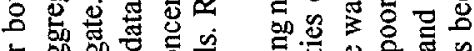

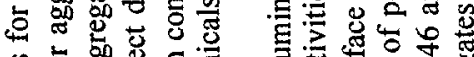

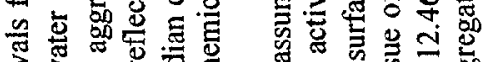

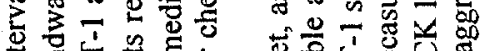

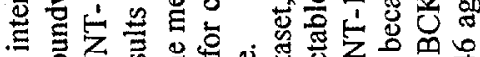

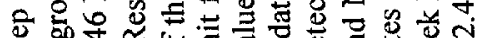

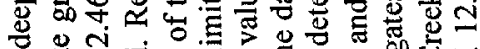

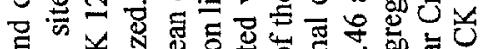

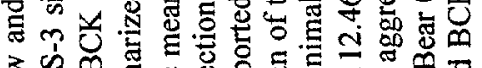

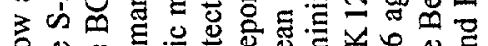

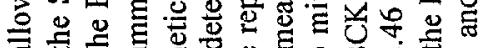

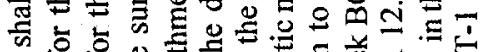

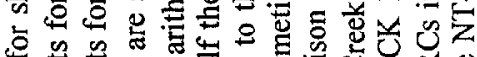

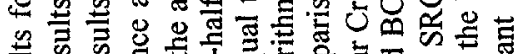

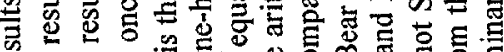

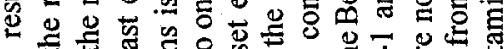

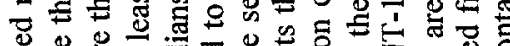

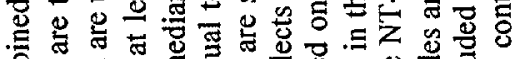

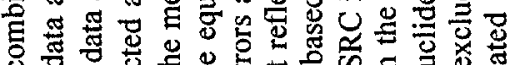
\&

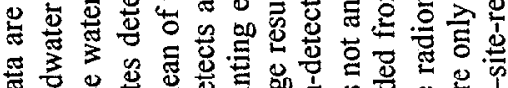

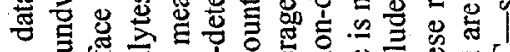

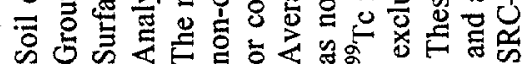


Table A-2. Results of chemical analysis of groundwater in the Maynardville Limestone and Bear Creek FA and of surface water at SS-4, SS-5, and BCK 9.47

\begin{tabular}{|c|c|c|c|c|c|c|c|c|c|c|c|c|c|c|c|c|}
\hline \multirow{2}{*}{ 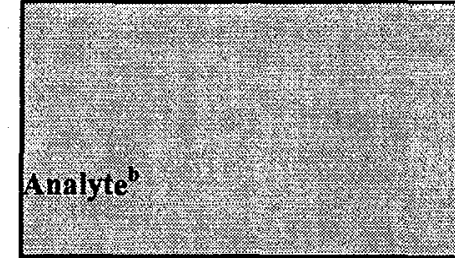 } & \multicolumn{4}{|c|}{ Groundwater } & \multicolumn{4}{|c|}{ 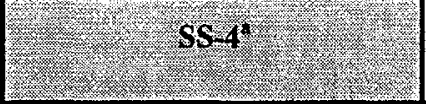 } & \multicolumn{4}{|c|}{ SS-5 $5^{\mathrm{a}}$} & \multicolumn{4}{|c|}{ Bear Creek at BCK $9.7^{\circ}$} \\
\hline & $\begin{array}{l}\text { Freq. of } \\
\text { wells w. } \\
\text { detects }\end{array}$ & Max & $\begin{array}{c}\text { Mean of } \\
\text { the } \\
\text { medians }\end{array}$ & SRC? & $\begin{array}{l}\text { Wreq of } \\
\text { cerrot }\end{array}$ & Max & $\left(\begin{array}{l}\text { Avge } \\
\text { result }\end{array}\right.$ & SRO? & $\begin{array}{c}\text { Freq. of } \\
\text { detect }\end{array}$ & $\begin{array}{c}\text { Max } \\
\text { detect }\end{array}$ & $\begin{array}{l}\text { Avg. } \\
\text { result }\end{array}$ & SRC? & $\begin{array}{l}\text { magrat } \\
\text { deregt }\end{array}$ & $\operatorname{Max}_{\text {detect }}$ & $\int \begin{array}{l}\text { Avg } \\
\text { result }\end{array}$ & \\
\hline \multicolumn{17}{|c|}{ Inorganics $(\mathrm{mg} / \mathrm{L})$} \\
\hline Aruninin & $78 / 78$ & 68 & 0.3 & & 19127 & 2.98 & 0,34 & & $17 / 27$ & 0.813 & 0.098 & & 261.35 & 298 & 1.68 & \\
\hline Antimpoy & $1 / 78$ & 0.13 & 0.027 & & 1113 & 00028 & 10.001 & & 1/ 13 & 0.003 & 0.001 & & Pr? & 1003 & 100001 & \\
\hline Arsenic & $1 / 17$ & 0.005 & 0.0025 & & & & & & & & & & 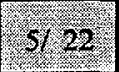 & 0.023 & 0003 & \\
\hline Barium & $78 / 78$ & 10 & 0.23 & & 27127 & 0.24 & 0.19 & & $27 / 27$ & 0.12 & 0.083 & & 33135 & 0.204 & 0.099 & \\
\hline Bergllimin & $40 / 78$ & 0.0039 & 0.0002 & & & & & & $1 / 27$ & 0.0003 & 0.0002 & & 1135 & 0.0015 & 0.0002 & \\
\hline Boron & $78 / 78$ & 3 & 0.16 & $\mathrm{Y}$ & $1 4 \longdiv { 1 4 }$ & 0.26 & 0.11 & Y & $13 / 14$ & 0.18 & 0.075 & $\mathrm{Y}$ & $13 \% 13$ & 4.1 & 0.80 & Y \\
\hline Cadmium & $21 / 82$ & 1.0200 & 0.008 & & & & & & & & & & & & & \\
\hline Calcum & $82 / 82$ & 880 & 119.52 & & 27.27 & 140 & 100,2 & & $27 / 27$ & 87 & 62.73 & & 35135 & 101 & 64.67 & \\
\hline Chromium & $39 / 82$ & 0.71 & 0.007 & & & & : & & & & & & 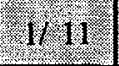 & 0.01 & 0.006 & \\
\hline Cobalt & $17 / 78$ & 0.140 & 0.003 & & & 2 & $\sqrt{120}$ & & & & & & $\sqrt{3.35}$ & 0.014 & 10.002 & \\
\hline Copper. & $70 / 78$ & 0.19 & 0.005 & Y & $4 \sqrt{27}$ & 0.007 & 0.002 & & $2 / 27$ & 0.007 & 0.002 & & 1735 & 0.003 & 0.003 & \\
\hline ron & $78 / 78$ & 170 & 1.82 & & $23 / 27$ & 3.72 & 0.46 & & $20 / 26$ & 1.13 & 0.111 & & $34 / 35$ & 38 & 2.39 & \\
\hline Head & $61 / 82$ & 0.23 & 0.0046 & $\mathrm{Y}$ & 11.2 & 0.005 & 0.002 & & $1 / 12$ & 0.012 & 0.003 & & & & $\sqrt{3}+3$ & \\
\hline Eithimin & $5 / 6$ & 0.085 & 0.029 & $\mathrm{Y}$ & 717 & 0.036 & 0,029 & & $7 / 7$ & 0.014 & 0.010 & & 7.7 & 0.091 & 0.060 & \\
\hline Magnesium & $82 / 82$ & 250 & 28.58 & & 27127 & 26 & 20.27 & & $27 / 27$ & 20 & 15.34 & & $35 t: 35$ & 18.1 & 11.44 & \\
\hline Manganese & $78 / 78$ & 16 & 0.37 & $\mathrm{Y}$ & 26127 & 0.809 & 0.14 & 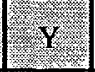 & $21 / 27$ & 0.048 & 0.007 & & $34 \times 35$ & 0.708 & 0.126 & \\
\hline ren & & & & & \%? & -2. & ras & 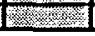 & & & & & खi: & 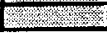 & Din & \\
\hline
\end{tabular}




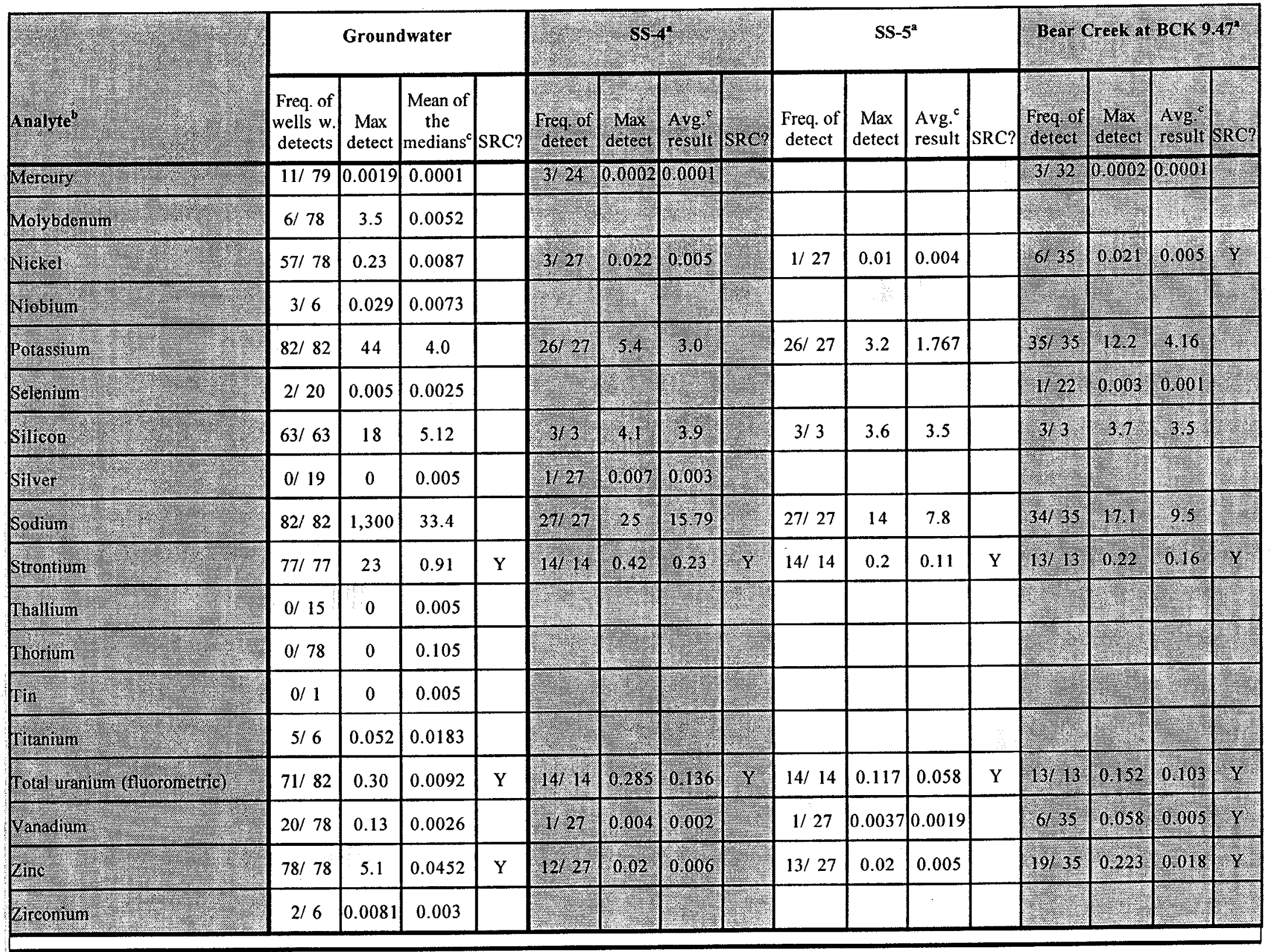




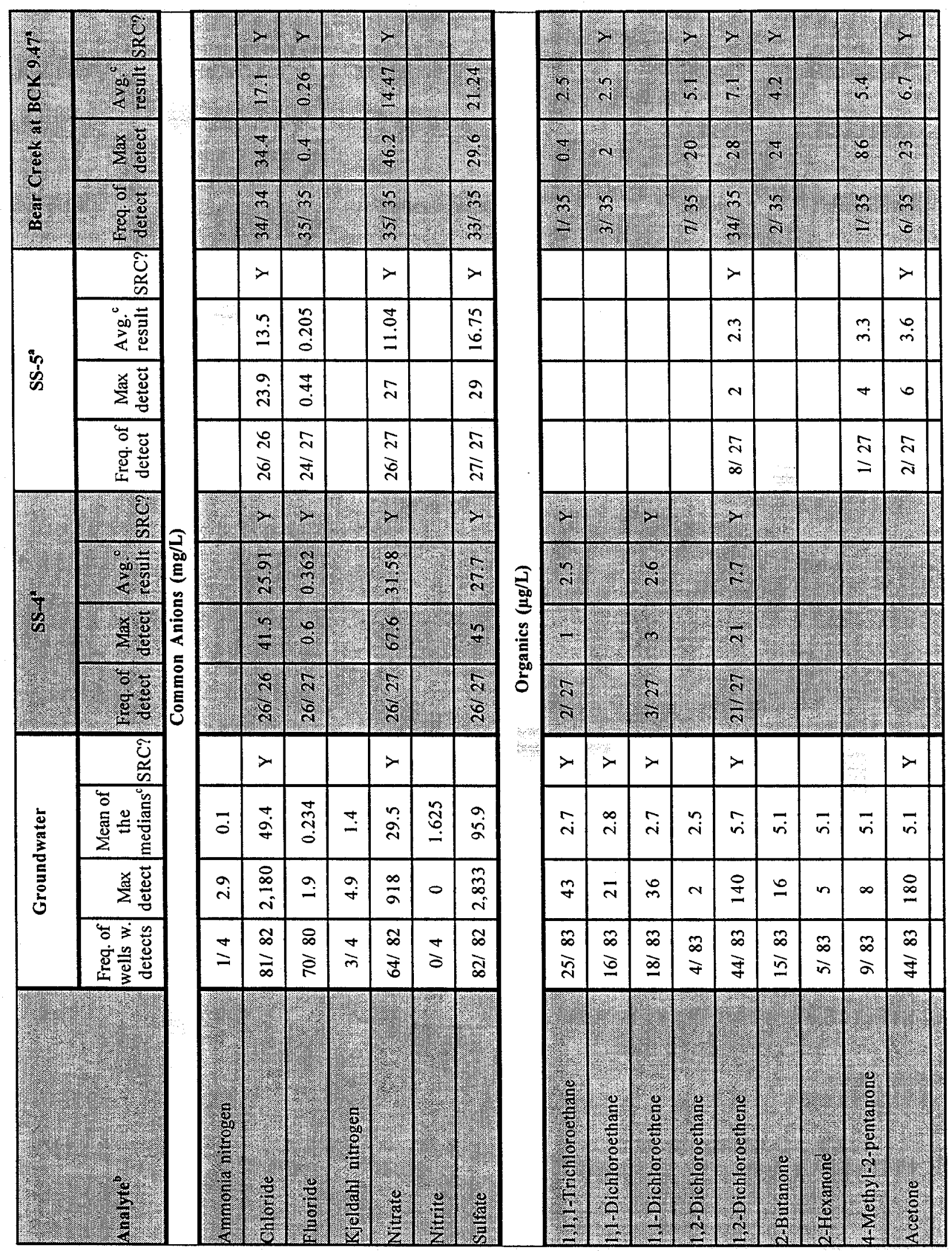




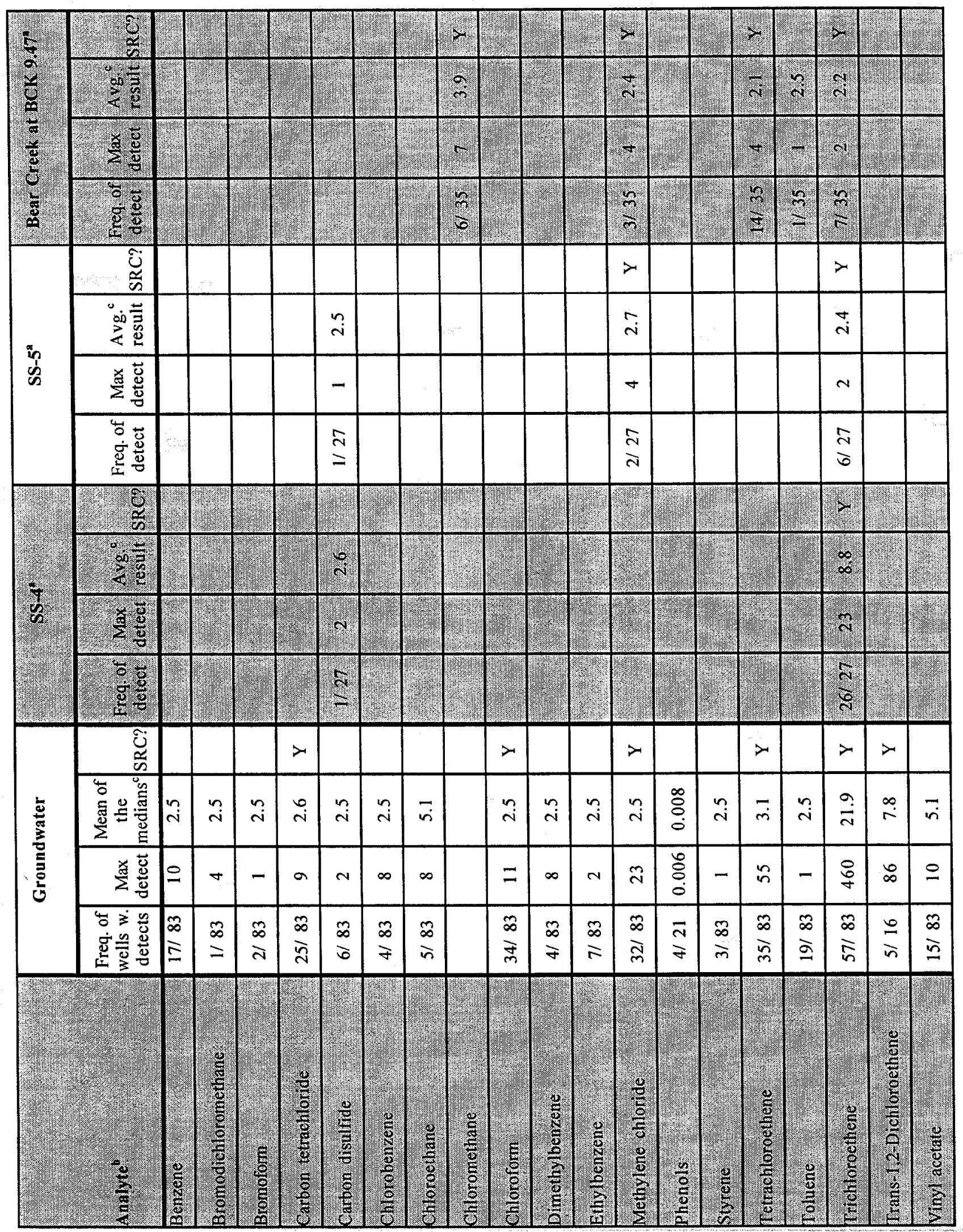




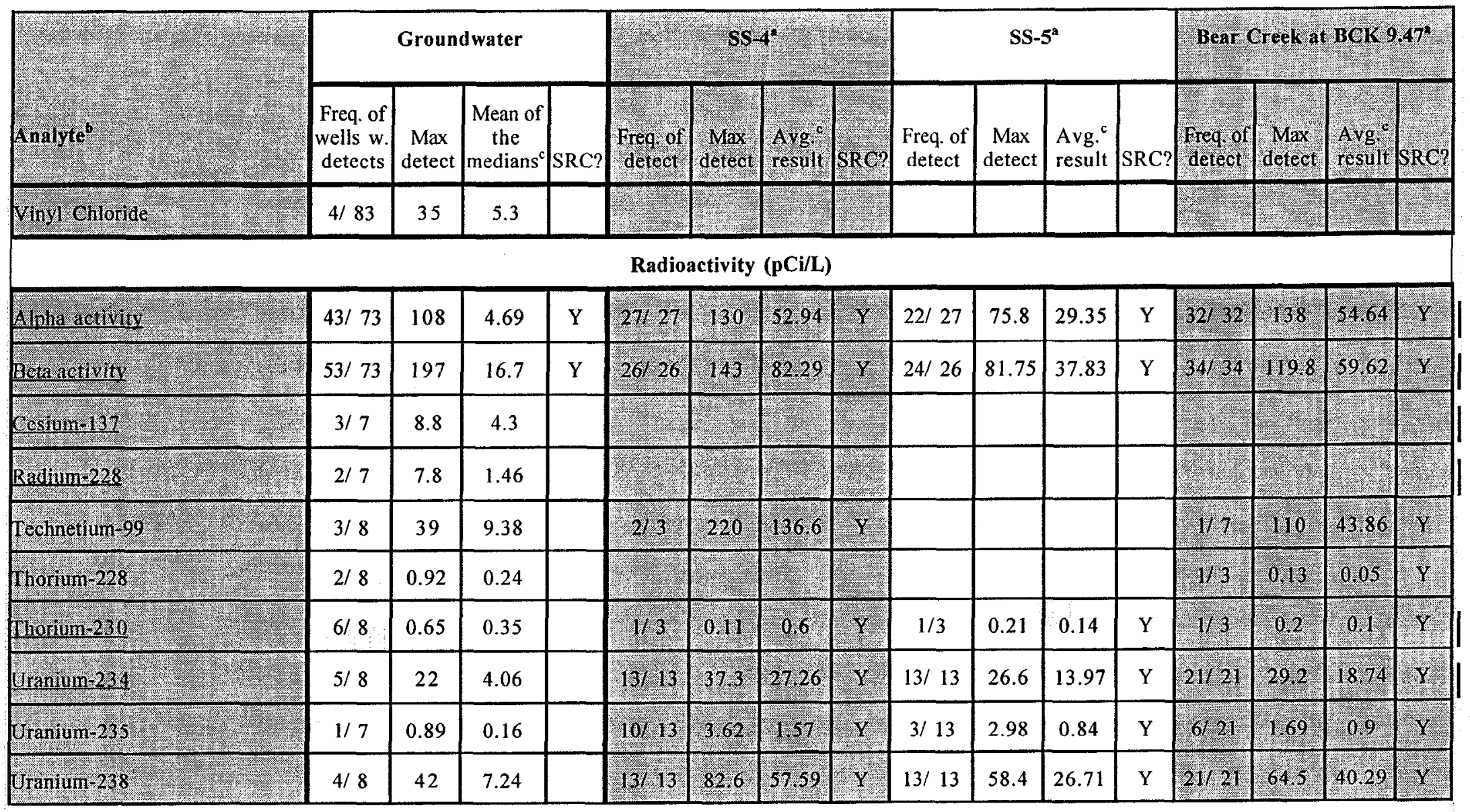

${ }^{a}$ Groundwater data are the results for the SS-4, SS-5, and BC at BCK 9.47. 114.

$b$ Analytes detected at least once are summarized. Results reflect data validation and data usability screens.

$c$ The mean of the medians is the arithmetic mean of the median concentration of an analyte for each well. In calculating the mean of the medians it was assumed that non-detects are equal to one-half the detection limit for chemicals. Radionuclide data qualified as non-detect based on comparison to minimal detectable activities or counting errors are set equal to the reported value. 
Table A-3 Groundwater analytical results from the reactive barrier sites

\begin{tabular}{|c|c|c|c|c|c|c|c|c|c|c|}
\hline \multirow{2}{*}{$\begin{array}{l}\text { Sample No. (location) } \\
\text { Collection date } \\
\text { Analysis }\end{array}$} & \multicolumn{4}{|c|}{$\begin{array}{c}\text { Pathway } 3 \text { (GW-834) } \\
8 / 20196\end{array}$} & \multicolumn{2}{|c|}{$\begin{array}{c}\text { Pathway } 2 \text { (GW-835) } \\
8 / 14 / 96\end{array}$} & \multicolumn{4}{|c|}{$\begin{array}{c}\text { Pathway } 1 \\
8 / 19 / 96\end{array} \quad(G W-837)$} \\
\hline & Rep I & $\begin{array}{l}\text { ered } \\
\text { Rep } 2\end{array}$ & $\begin{array}{l}\text { Unfilter } \\
\text { Rep I }\end{array}$ & d $\operatorname{Rep} 2$ & $\begin{array}{l}\text { Filtered } \\
\text { Rep I }\end{array}$ & $\begin{array}{l}\text { Unfiltered } \\
\text { Rep I }\end{array}$ & Rep I Filter & Rep 2 & Rep I Unfilt & ed 2 \\
\hline Bicarbonate, $\mathrm{mg} /$ & & 443 & 4 & & & 434 & & & & \\
\hline Mercury, mg/L & 0.0002 & & $<0.0002$ & & & 0.00049 & 0.00039 & & 0.0012 & \\
\hline Aluminum, ugh & 2760 & 2670 & 9860 & 8210 & 1220 & 4550 & 4690 & 4630 & 27900 & 25500 \\
\hline Boron, ug/L & 102 & $<100$ & 114 & $<100$ & 108 & 108 & 23.1 & $<100$ & 39.9 & $<100$ \\
\hline Barium, ugh & $>23200$ & 23800 & 23700 & 24300 & 83.5 & 95.2 & 483 & 489 & 578 & 587 \\
\hline Calcium, ug/L & $>1000000$ & 2629000 & $>1000000$ & 2662000 & 163000 & 163000 & $>1000000$ & 1647000 & $>1000000$ & 1681000 \\
\hline Cadmium, ug/L & 411 & 391 & 427 & 404 & $<20$ & $<20$ & 199 & 184 & 204 & 196 \\
\hline Cobalt, ug $\mathrm{L}$ & 216 & 212 & 222 & 211 & $<20$ & $<20$ & 257 & 250 & 265 & 265 \\
\hline Chromium, ug/L & $<20$ & $<100$ & $<20$ & $<100$ & $<20$ & $<20$ & $<20$ & $<100$ & $<20$ & $<100$ \\
\hline Copper, ugh & 65.2 & $<200$ & 70.5 & $<200$ & $<30$ & $<30$ & 58.6 & $<200$ & 77.2 & $<200$ \\
\hline Iron, ug/L & 362 & $<800$ & 5810 & 5700 & 712 & 4580 & 207 & $<800$ & 14300 & 14300 \\
\hline Potassium. ug/L. & 29000 & 26500 & 32000 & 28200 & 6380 & 7300 & 43200 & 38800 & 47000 & 42400 \\
\hline Magnesium, ug/L & 335000 & 322000 & 343000 & 327000 & 26000 & 26400 & 152000 & 146000 & 158000 & 151000 \\
\hline Manganese, ugh & $>100000$ & 145000 & $>100000$ & 147000 & 1120 & 1160 & 100000 & 157000 & $>100000$ & 159000 \\
\hline Molybdenum, ug/L & $<40$ & $<200$ & $<40$ & $<200$ & $<40$ & $<40$ & $<40$ & $<200$ & $<40$ & $<200$ \\
\hline Sodium, ug/L & 506000 & 494000 & 516000 & 500000 & 17400 & 17300 & 695000 & 677000 & 705000 & 688000 \\
\hline Nickel, ug/L & 1660 & 1710 & 1710 & 1750 & $<40$ & $<40$ & 2130 & 2130 & 2140 & 2210 \\
\hline Lead, ugh & $<200$ & $<800$ & $<200$ & $<800$ & $<200$ & $<200$ & $<200$ & $<800$ & $<200$ & $<800$ \\
\hline Thallium, ugh & 136 & $<500$ & 139 & $<500$ & $<100$ & $<100$ & 143 & $<500$ & 169 & $<500$ \\
\hline Vanadium. ug/L & $<10$ & $<50$ & $<10$ & $<50$ & $<10$ & $<10$ & $<10$ & $<50$ & $<10$ & $<50$ \\
\hline
\end{tabular}


Table A-3 (continued)

\begin{tabular}{|c|c|c|c|c|c|c|c|c|c|c|}
\hline \multirow{2}{*}{$\begin{array}{c}\text { Sample No. (location) } \\
\text { Collection date } \\
\text { Analysis }\end{array}$} & \multicolumn{4}{|c|}{$\begin{array}{c}\text { Pathway } 3 \text { (GW-834) } \\
8 / 20 / 96\end{array}$} & \multicolumn{2}{|c|}{$\begin{array}{c}\text { Pathway } 2 \text { (GW-835) } \\
8 / 14 / 96\end{array}$} & \multicolumn{4}{|c|}{ Pathway ${ }_{8 / 19 / 96}^{1}$ (GW-837) } \\
\hline & Rep I & $\begin{array}{l}\text { Filtered } \\
\text { Rep } 2 \\
\end{array}$ & Rep I & $\begin{array}{l}\text { ed } \\
\text { Rep } 2\end{array}$ & $\begin{array}{l}\text { Filtered } \\
\text { Rep I } \\
\end{array}$ & $\begin{array}{l}\text { Unfiltered } \\
\text { Rep I }\end{array}$ & $\begin{array}{r}\text { Filtered } \\
\text { Rep 2 } \\
\end{array}$ & Rep I & & $\begin{array}{c}\text { Unfiltered } \\
\text { Rep 2 }\end{array}$ \\
\hline Zinc, ug/L & 33.5 & $<100$ & 44.8 & $<100$ & $<20$ & $<20$ & 97.6 & $<100$ & 119 & 105 \\
\hline Selenium, ug/L & $<500$ & $<3000$ & $<500$ & $<3000$ & $<500$ & $<500$ & $<500$ & $<3000$ & $<500$ & $<3000$ \\
\hline Sitver, ug/L & $<20$ & $<80$ & $<20$ & $<80$ & $<20$ & $<20$ & $<20$ & $<80$ & $<20$ & $<80$ \\
\hline Arsenic, ug/L & $<100$ & $<500$ & $<100$ & $<500$ & $<100$ & $<100$ & $<100$ & $<500$ & $<100$ & $<500$ \\
\hline Beryllium, ug/L & 2 & $<5.0$ & 2.5 & $<5.0$ & $<1.0$ & $<1.0$ & 2.9 & $<5.0$ & 4.5 & $<5$ \\
\hline Antimony, ug/L & $<100$ & $<500$ & 100 & $<500$ & $<100$ & $<100$ & $<100$ & $<500$ & $<100$ & $<500$ \\
\hline Lithium, ug $/$ L & 41.8 & $<50$ & 50.5 & $<50$ & 30.6 & 32.4 & 119 & 93.8 & 143 & 124 \\
\hline Phosphorous,ug/L & 547 & $<2000$ & 668 & $<2000$ & $<300$ & $<300$ & 562 & $<2000$ & 724 & $<2000$ \\
\hline Titanium, ug/L & $<20$ & $<80$ & 189 & 96.2 & $<20$ & 124 & $<20$ & $<80$ & 244 & 161 \\
\hline Strontium, ug/L & 660 & 6770 & 6670 & 6850 & 488 & 490 & 2390 & 2440 & 2450 & 2500 \\
\hline Alkalinity, mg/L & & & 420 & & & 310.1 & & & 410 & \\
\hline Alpha activity, pC/L & & & 210 & & & 370 & & & 790 & \\
\hline Beta activity, pCl & & & 9000 & & & 280 & & & 4300 & \\
\hline Tritium, $\mathrm{pC} / \mathrm{L}$ & & & 1500 & & & 46 & & & 1100 & \\
\hline Fluoride, $\mathrm{mg} / \mathrm{L}$ & & & 4.8 & & & 0.4 & & & 4.9 & \\
\hline Chloride. mg/L & & & 260 & & & 28 & & & 320 & \\
\hline Nitrite as Nitrogen, mg/L & & & 8.3 & & & 0.2 & & & 7.3 & \\
\hline Bromide, mg/L & & & NA & & & NA & & & NA & \\
\hline Nitrate as Nitrogen, $\mathrm{mg} / \mathrm{L}$ & & & 2300 & & & 29 & & & 1400 & \\
\hline
\end{tabular}


Table A-3 (continued)

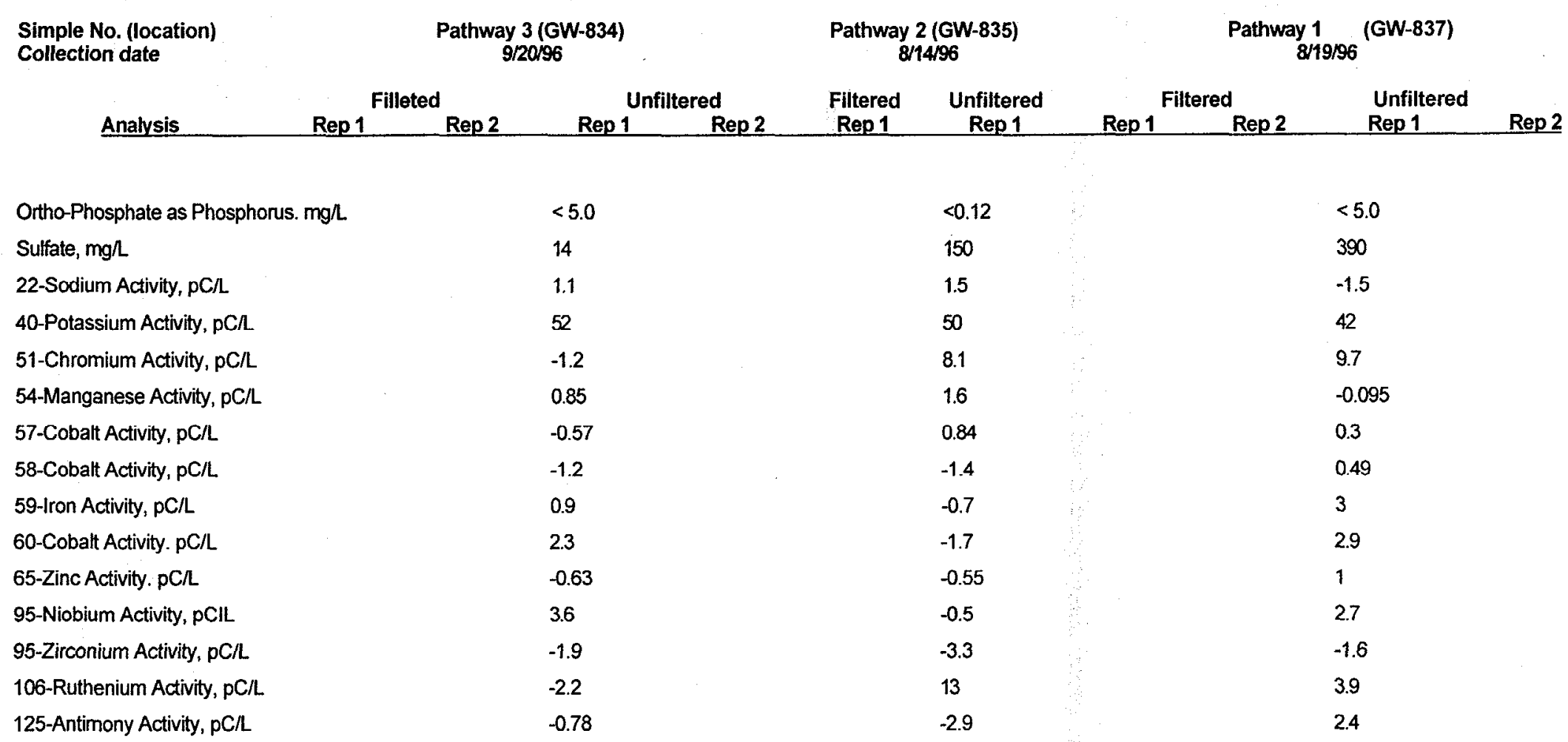


Table A-3 (continued)

\begin{tabular}{|c|c|c|c|c|c|c|c|}
\hline $\begin{array}{l}\text { Sample No. (location) } \\
\text { Collection date }\end{array}$ & Pathwa & $\begin{array}{l}\text { GW-834) } \\
96\end{array}$ & $\begin{array}{r}\text { Pathway } \\
8\end{array}$ & $\begin{array}{l}\text { GW-835) } \\
196\end{array}$ & Pathway & ${ }_{196}^{(G W-837)}$ & \\
\hline & Filleted & Unfiltered & Filtered & Unfiltered & Filtered & Unfiltered & Dan? \\
\hline Analysis & $\operatorname{Rep} 1$ & $\operatorname{Rep} 1 \quad \operatorname{Rep} 2$ & $\operatorname{Rep} 1$ & $\operatorname{Rep} 1$ & Rep 1 Rep 2 & $\operatorname{Rep} 1$ & Rep 2 \\
\hline 134-Cesium Activity, pC/L & & -1.2 & & -0.52 & & 1.2 & \\
\hline 137-Cesium Activity. pC/L. & & -0.77 & & -0.064 & & -2.4 & \\
\hline 144-Cerium Activity, $\mathrm{pC} / \mathrm{L}$ & & 1.3 & & -3.5 & & -2.1 & \\
\hline 208-Thallium Activity, pC/L & & 4.5 & & 3.4 & & 3.1 & \\
\hline 212-Bismuth Activity. pC/L & & 13 & & 13 & & 1.8 & \\
\hline 212-Lead Activity. $\mathrm{pC} / \mathrm{L}$ & & 18 & & 6.4 & & 12 & \\
\hline 235 Uranium Activity, $\mathrm{pC} / \mathrm{L}$ & & 7.8 & & 5 & & 20 & \\
\hline Technetium-99, pC/L & & 22000 & & 150 & & 10000 & \\
\hline Total Dissolved Solids, mg/L & & & & 690 & & 11000 & \\
\hline Total Strontium, $\mathrm{pC} / \mathrm{L}$ & & -2.4 & & 0.4 & & 360 & \\
\hline Total Suspended Solids, mg/L & & 190 & & 9 & & & \\
\hline Uranium, $\mathrm{mg} / \mathrm{g}$ & & 0.0037 & & 1.7 & & 2.6 & \\
\hline Unknown, ug/L & & 65 & & & & $\infty 0$ & \\
\hline Acetaldoxime, ugh & & 6 & & & & & \\
\hline 1,2-Ethanediol, Dinitrate, ug/L & & 16 & & & & & \\
\hline
\end{tabular}


Table A-3 (continued)

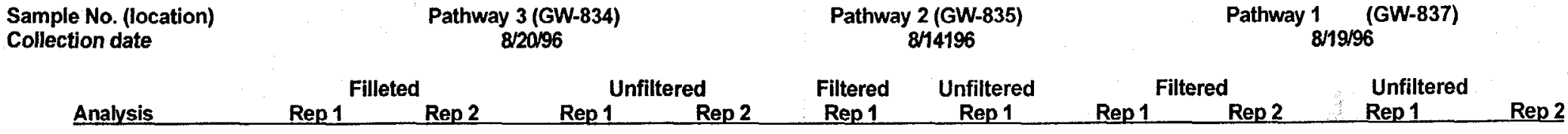

1,5-Pentanediol, Dinitrate, ug/

Chloromethane, ug $\Lambda$

Bromomethane, ug $/$ L

10

Vinyl chloride, ug/L

$10 \mathrm{U}$

$4 \mathrm{~J}$

$10 \mathrm{U}$

Chloroethane, ugh

Trichlorofluoromethane, ug/L

$10 \mathrm{U}$

$10 U$

Methylene chloride, ug $\mathrm{L}$

Acetone, ugh $L$

1,1,2-Trichloro

14

12

$10 \mathrm{U}$

1,2,2-trifiuoroethane, ug/

Carbon disulfide, ugh

1.1-Dichloroethene, ug/L

1,1-Dichloroethane, ug/L

cis-1,2-Dichloroethene, ug/L

tran-1,2-Dichloroethene, ug/L

Chloroform, ug/L

1,2-Dichloroethane, ug/h

2-Butanone, ug/L

100


Table A-3 (continued)

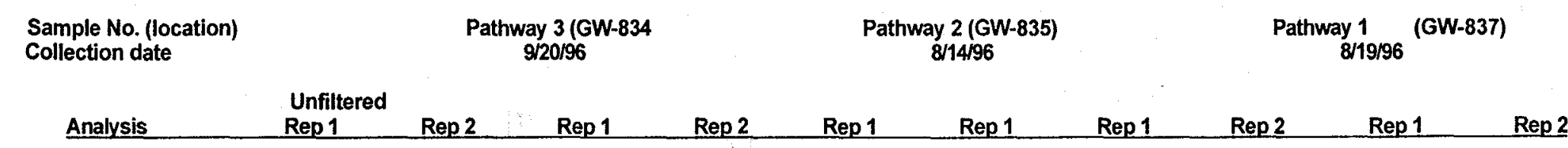

$\begin{array}{lccc}\text { 1,1,1-Trichloroethane, ug/ } & 10 \mathrm{U} & 10 \mathrm{U} & 11 \\ \text { Carbon tetrachloride, ug/ } & 10 \mathrm{U} & 10 \mathrm{U} & 10 \mathrm{U} \\ \text { Bromodichloromethane, ug/ } & 10 \mathrm{U} & 10 \mathrm{U} & 10 \mathrm{U} \\ \text { 1,2-Dichloropropane, ug/L } & 10 \mathrm{U} & 10 \mathrm{U} & 10 \mathrm{U} \\ \text { cis-1,3-Dichloropropene, ug/L } & 10 \mathrm{U} & 10 \mathrm{U} & 10 \mathrm{U} \\ \text { Trichloroethene, ug/L } & 10 \mathrm{U} & 10 \mathrm{U} & 10 \mathrm{U} \\ \text { Dibromochloromethane, ug/L } & 10 \mathrm{U} & 10 \mathrm{U} & 10 \mathrm{U} \\ \text { 1,1,2-Trichloroethane, ug/L } & 10 \mathrm{U} & 10 \mathrm{U} & 10 \mathrm{U} \\ \text { Benzene, ug/L } & 10 \mathrm{U} & 10 \mathrm{U} & 10 \mathrm{U} \\ \text { trans-1,3-Dichloropropane, ug/L } & 10 \mathrm{U} & 10 \mathrm{U} & 10 \mathrm{U} \\ \text { Bromoform, ug/L } & 10 \mathrm{U} & 10 \mathrm{U} & 10 \mathrm{U} \\ \text { 4-Methyl-2-pentanone, ug/L } & 10 \mathrm{U} & 10 \mathrm{U} & 1 \mathrm{~J} \\ \text { 2-Hexanone, ug/L } & 10 \mathrm{U} & 10 \mathrm{U} & 10 \mathrm{U} \\ \text { Tetrachloroethene, ug/L } & 2 \mathrm{~J} & 4 \mathrm{~J} & 14 \\ \text { 1,1,2.2-Tetrachloroethane, ug/L } & 10 \mathrm{U} & 10 \mathrm{U} & 10 \mathrm{U}\end{array}$


Table A-3 (continued)

Sample No. (location)

Collection date

Analysis

Toluene, ug/

Chlorobenzene, ugh

Ethylbenzene, ug/

Styrene, ug/

Xylene, ugh

Ethane

1,2-Dichloro-1,1,2-tr, ug/L
Pathway 2 (GW-835)

8/14/96

Pathway 3 (GW-834) $8 / 20 / 96$

Filtered

Rep 1

$\operatorname{Rep} 2$

Unfiltered

Rep 2

Rep 1

$\operatorname{Rep} 1$

$\operatorname{Rep} 1$

Rep 2

$\operatorname{Rep} 1$

$\operatorname{Rep} 2$

$10 \mathrm{U}$

$10 \mathrm{U}$

$10 \mathrm{U}$

$10 \mathrm{U}$

$10 \mathrm{U}$

$10 \mathrm{U}$

4.03
Pathway 1 (GW-837)
$10 \mathrm{U}$

$10 \mathrm{U}$

$10 \mathrm{U}$

$10 \mathrm{U}$

100

$10 \mathrm{U}$ 


\section{Appendix B}

\section{MATERIAL SAFETY DATA SHEETS}




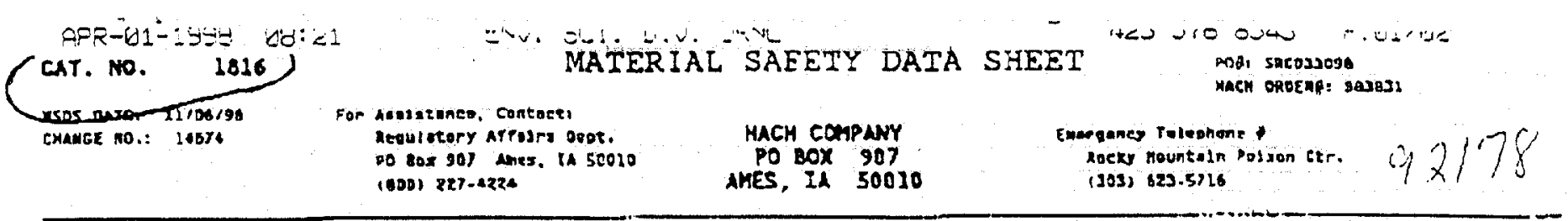

\section{PRDDUCT IBENTIFICATION}

propuer xane, sulfide i geagent

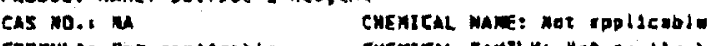

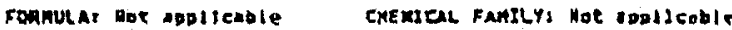

ras nuHatR: MaE2 13

\section{IMGREDIENTS}

Sutruate Aes:

PCT: 485

TLY: $1 \rightarrow \operatorname{lng}$

CAS NO.1 2664-9J-9 SARA, MOT LESTEO IUAC, 1 ISTEO

PELI I Ag/MJ

Halaabi Cause severe Durns. Xarnrul if Inholwo. Knaen carclnogen

Ot her coupenent

PETI LI CAS MO.: MA SAMAY LOT LISTEO

TLYi NoC uppitedite

Marapoi Not osplicable

PEL, Not uppllcable

Demineralixed Vator

PET, to 100 CRS nO.: 1732-18.5 SARA NOT LISTED

rLY, war appliegele PEL Mor opplizebil

razaros none

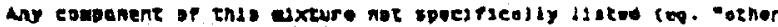

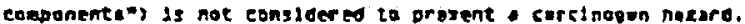

\section{PKYSICAL DATA}

STATE, IJquLd Appeagance, Cicar, colarless of lloht brown odad, Hono

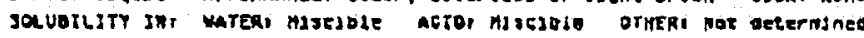

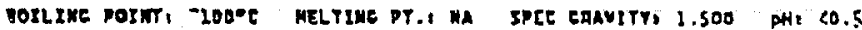

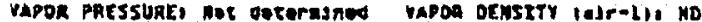

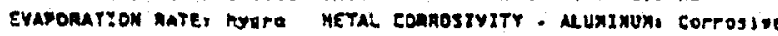

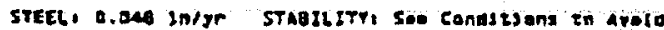

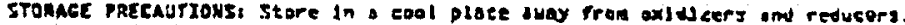

\section{FIRE, EXPLOSION HAZARD AHD REACTIVITY DATA}

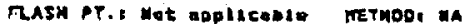

RAMTABILITY LMSTS - LOWERT NA UPPERI WA

SUSEEPTIERT ITY TO SPOMTAKEOUS MEATIME: Mane

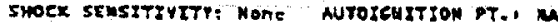

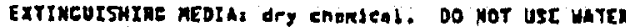

FiRejexplosion mazarosi noy ente toxse funet in life

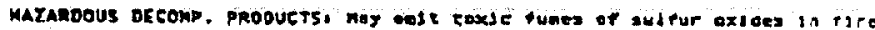

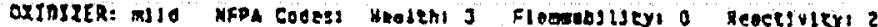
AFPA smbol,

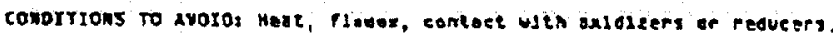

cousties and countse fumen, septie seld, ehlorasulfonic acto

\section{MEALTH HAZARD DATA}

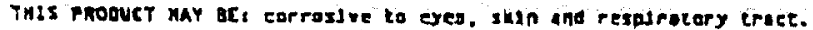

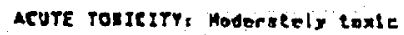
koutes or crrosuat: ingeatson, snhwiation TARSET ONGAMSi Not totorol nod

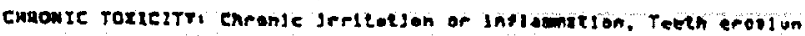
ROUTES OF EXPOSURE: Inhelstion Theer oxesus, toath, lungs

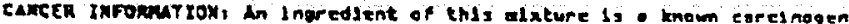

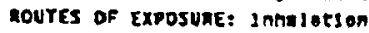

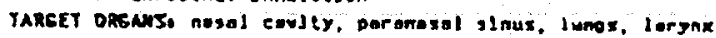

OVEAEXPOSURE, Sovarely burns any tsadue comtacted. Ireathine in the sl at or

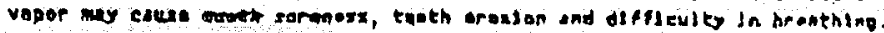
Inniatation of the mble or vapor of cone. subfurle beld ean chuse eancer of tho nases tsegue. Lunes amo larynx.

MEDICAL COMOKTIONS AGGRAVATEO BY EXPOSUREi Pra-mistIm oyn, skin and -espiratory conditions

\section{PRECAUTICHARY HEASURES}

Avald eantece with fyes, shin and clothing

Do net oreatine mist or yopor.

Wosh snoroughiy ofter hand Jans.

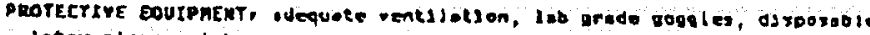
latar lover, lod coet

\section{FIRST AID}

EYE AMD SKIn EOnIACT, Imedinethy flush ores and aln ulen woter par 15 manutes. Memove concosinated clothine. Call physicsan.

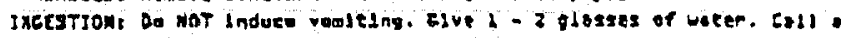

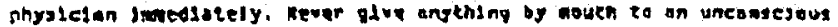
peroon.

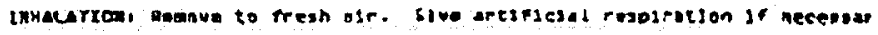
cell ohyriedan.

\section{SPILL AND DISPOSNL PROCEDURES}

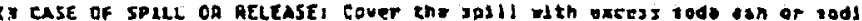

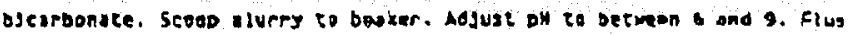

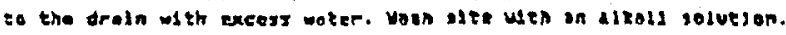
Dispose of II ACEOHDANEE UITH ALL FEDERAL, STATE, MD LOCAL REgULATIONS.

\section{TRANSPORTATION DATA}

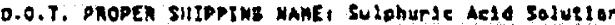

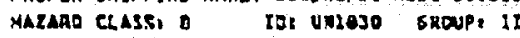

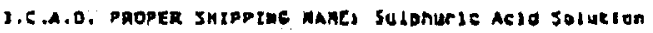
Mazapo Classi a lo, unleso eroupt it

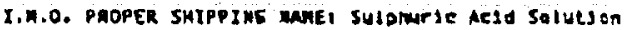

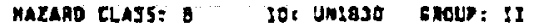

\section{$x$. REFERENCES}

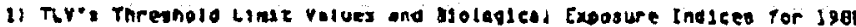

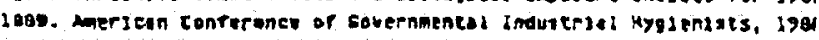

(i) Alr Contoulnonty. Federal Ragjator, Vol. S4, Wo. 12, Thursday, Januar 19. 2989. Op. RJj2-R983.

11 In-nouse intorsazton

i) rechndes Judgment

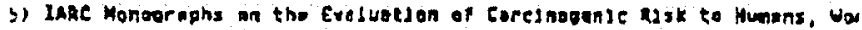

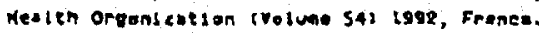

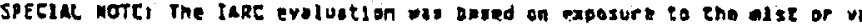

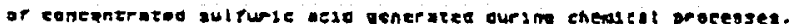

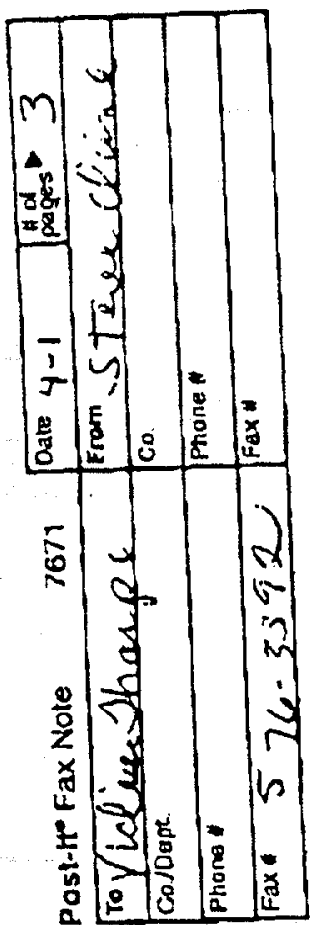




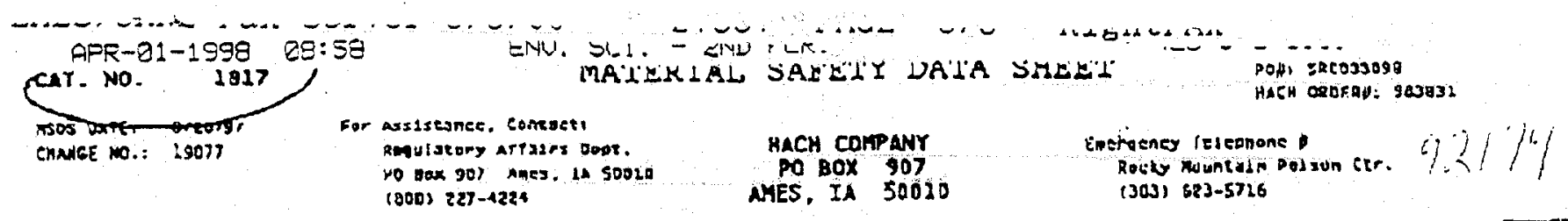

\section{PRODUCT IDENTIFICATION}

PROOUCT MANE: SWIfloe 2 Rezgent

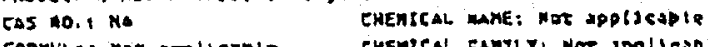

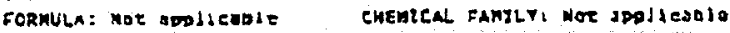

nSOS munaER: N00435

\section{INGREDIENTS}

Dotanesum Dishromete

$$
\begin{aligned}
& \text { PET: Cl CAS NO.1 M7PA.50.9 SORAI LESTEO } \\
& \text { YLV: } 3.05 \mathrm{mg} / \mathrm{hJ} \text { os Ge PELI } 0.1 \mathrm{mg} / \mathrm{m})(F(V I) \\
& \text { TARC: LISTEO WTD: LISTEO }
\end{aligned}
$$

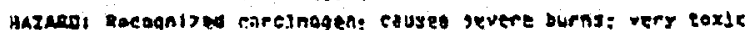

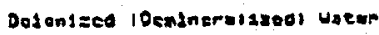

$$
\begin{aligned}
& \text { PCT! ro } 100 \text { CAS WO, 7732-28-5 SARA: NOT LISTED } \\
& \text { TLY: Not Opollicoble } \\
& \text { BAZARD: Wane }
\end{aligned}
$$

\section{PHYSICAL DATA}

5TATE: llanla agocallance: Clear. orbage ODOR: Mone

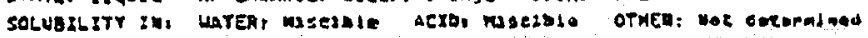

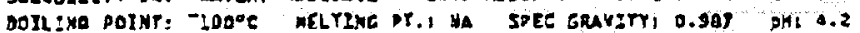

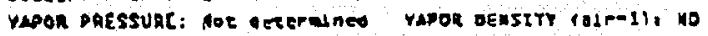

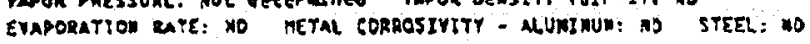
sTANTLITY, STHO LE

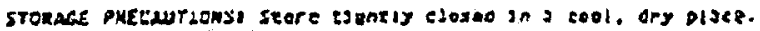

IV. FIRE, EXPLOSTON HAZARO ND REACTIVITY DATA

FLSA RT.8 Nat apdiseablo nethos: HA

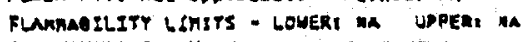

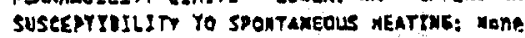

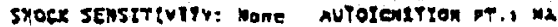

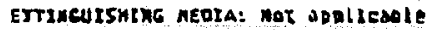

FIREJEXPLOSION MAZAADSI mone

Mazanoous oreonp. propuets: Wone

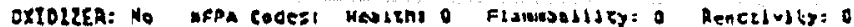

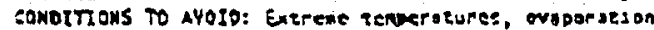

\section{MEALTH HAZARD DATA}

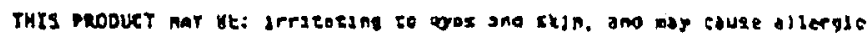
Ikin rosesion.

ACuTE TOXje:tr: proecleallig non-tade ROUTES af Exposunei wot sppliesole

Tancet organs: noe 10012 eods

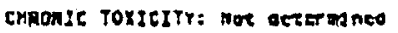
noutes of Exposure: Wot deteresned

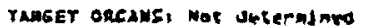

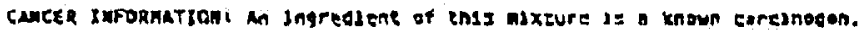
Routes of Exposune, janstsel on

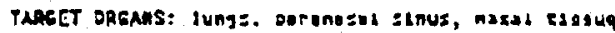

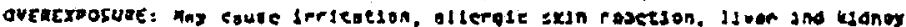
suagao.

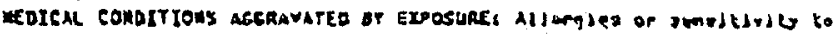
enromle ocso or ehromazes

\section{PRECAUTIONARY MERSURES}

araln contjet ujen eres and sudn.

Do nat orasente ofyt or eagor.

nesh enoravaniy orter hanolina.

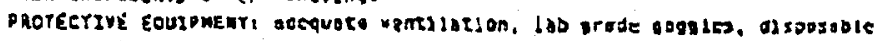
la:en gloves

\section{FIRST AID}

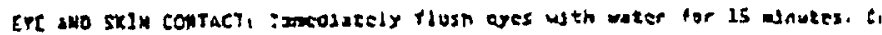

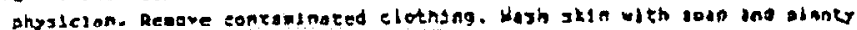
weer.

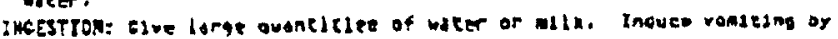

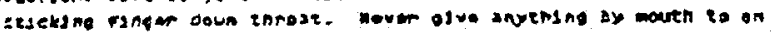
unconses ous parson. Esll phresessn.

IMHNASTSOW, Not sooliscoole

\section{SPILL NO OTSPOSAL PROCEDURES}

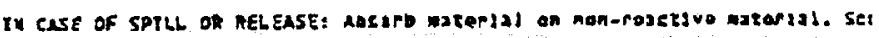
up the matarial ans nispese of In an EPA hoor ored nozarooux wate racli1ty. Docontowinase sito with o soup solweson.

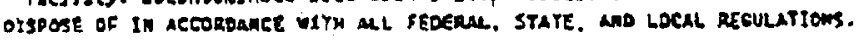

\section{TRANSPORTATION DATA}

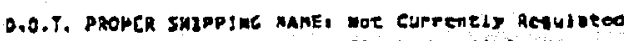
MAzARS CLASS: MA TO: MA SROUD: Wh

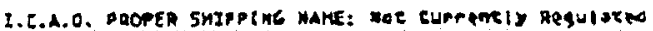

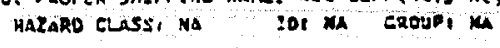

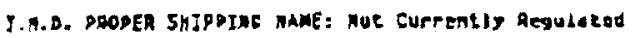
matano eluss: Wh Do: Ma Eneve: Ma

\section{$X$. REFERENCES}

1) In-house informatinn

c) Teenntesl suaqmest

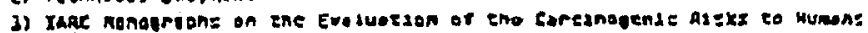

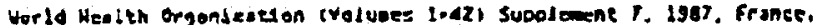

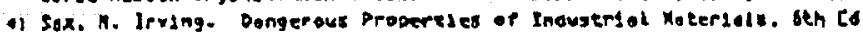
nev Yarki var wastrant Rosinnaje 60. 2984.

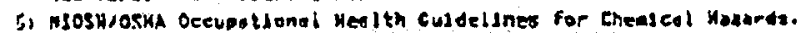
Efneinnirs: Deportaent of Health ard Human Servicee. 1901.

sual Thly broduct contalins. chewles or eneaseals suaject to the raport.

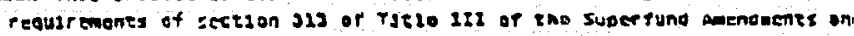
Aeduthorszation det of 1986 and 40 efR Part 372.

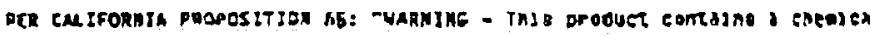

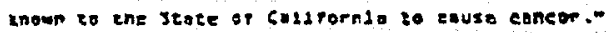




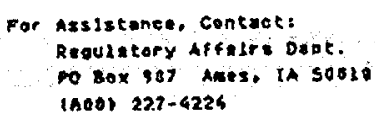

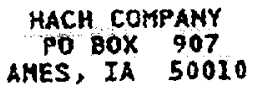

Esergenay iespphone Rscky Hountazn Polian ctr. (305) 623-57)

\section{PRODUKT IDENTIFICATION}

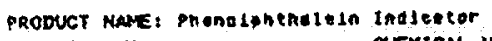

CAS NO. : HA Ciemteal have, Not opds sosalo

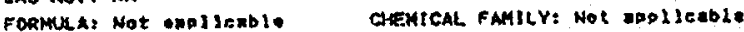
HSOS NLAKBER, MOLOES

\section{INGREDIENTS}

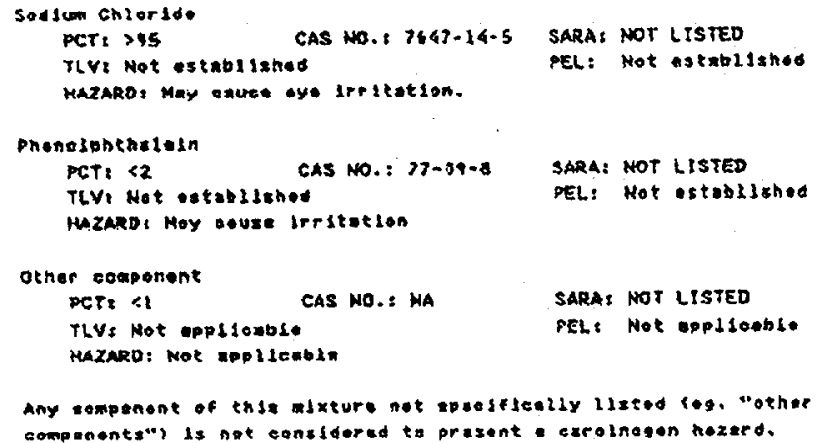

\section{PHYSICAL DATA}

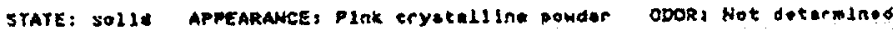
SOLUBILITY IN: WATER: SOLUb3E ACID: Not dothrminad

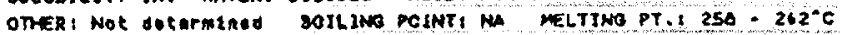

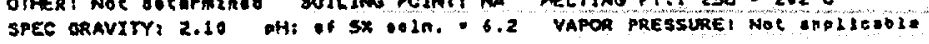
VAPOR DENSITY (AIRIII: NA EVAPORATION RATE: NA

METAL CORQOSIUTTY - ALLMINLME ND STEEL: NO STABLLITV: STEBI.

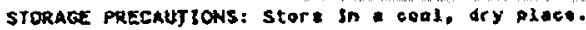

\section{FIRE, EXPLOSZON HAZARO AND REACTIVITY DATA}

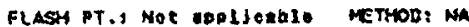

FLAMHABILSTY BIMTTS - LOWER! KA UPPER: MA

SUSCEPTIBRLITY TO SPOWTANEOUS HEAT ING I NOH:

SHOCX SEMSITIVITY: NONE AUTOTENITION PT.A NO EXT INOUISHING MEOLAI water

FIRE/EXPLOSION HazAROS: MaY ande ocrld snoke and fumes in fire

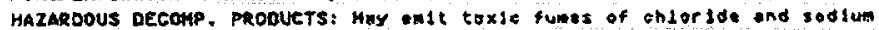

axide in firt.

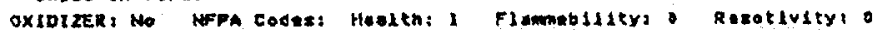

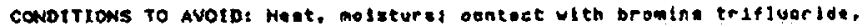

11tnium

\section{HEALTH HAZARD DATA}

This proouer may ae: ireltating to gyes and skin.

ACUTE TOXICITY: Moderatoly toxle ROUTES of Exposukt: Insertion TAROET OROANSt NOt delerwined

CHRONIC TOXICITY: Nat Hoternined

ROUTES OF Exposures Not determined

TARGET CRGANS: Nol diterninod

CAHCER THFCRAMTIOWI Not detarminad

RQUTES OF Exposine, Hot determinut

TARGE ORRANS! Not determintd

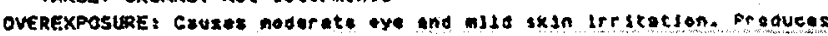

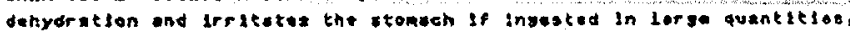
ouslng voniting. diaxrhea, nuscular twltehing and rlgldity, colkaso,

dazth, May sause blood oreszurs groblems.

meOCAL CONDETrOHS AGGRAVATEO BY EXPOSLRE: Pra-oxlstins oy condstlons.

\section{PRECAUTTONARY MEASURES}

Arosd cantact with eves ond skin.

mesh thorougniy after hendilas.

PROTECTIVE EqUIPHENT: Adecuate ventllation, lab grode sosgles

\section{FIRST AID}

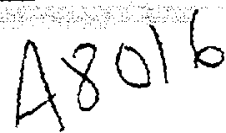

EVE AND SXIN CONTACT: Flush with whenty of watne.

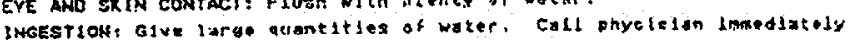
INHALATION: Romove to Gresh a!t.

\section{SPILL AND DISPOSAL PROCEDURES}

IN CASE of SPILL OR RELEASE; Sweop up nowder. AVolf brenthlag mater:a olsselve in water. Flush hown tive draln with ixesss katar.

OIs

\section{TRANSPORTATIOA DATA}

D. O.T. ROPER SMIPPING Nares Not Cus rontiy Regulated HAZARD CLASS, NA IDI NA GROUP: NA

I.C.A.O. PROPER SHIPPING NAKE: Not Currently Ragulated HAYARD CLASS: NA TOI NA GROUP: NA

X.M.O. PROPER SHJPPING HAHE: Nat CUFCanely RESULztan HazARO ClaSS: MA BD: NA GROMI: NA

\section{REFERENCES}

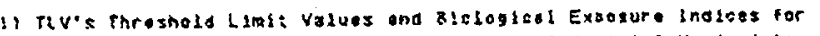
1989. Amer Jeen Confarence of covernmenxal industrlug Hyglenists,

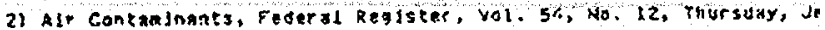
19, $198 \%$. p9. 2352-2703.

3) Lontus information

a) reennicol guogment

5) Aete Anot. 74: $121-124(1769)$

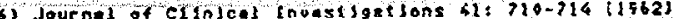

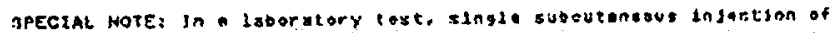

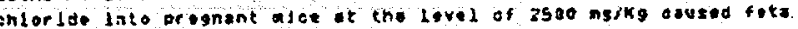
end maformatlans. In a laboratory tost, mienglven a 24 sasiun e: solution in olace nf or enking water dur ing pregnency produced nye b orult offsuring. 
For hasietence, contest: Reviletery Affelrs bint O Bax 107 ABgo. IA sinis

I. PRODUCT IDENTIFICATION

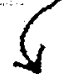

CATALOO NO.: 1637 CAs ino. 8 NA

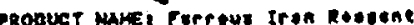
Fornutat Not sopitemb: REGARDINB THE ACCURACY OF 3MESE DATA OR THE RESULTS 10 OS OSTAINED FROH THE USE TISEREO

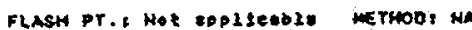

$r=0$

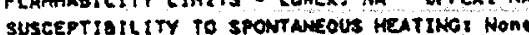

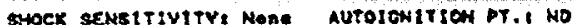

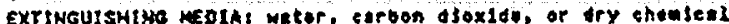

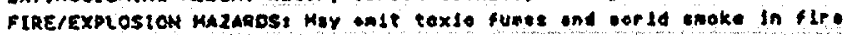

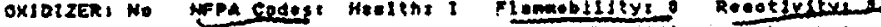

v. HEALTH HAZARD DATA

TIS PRODUCT MAY SEt Irrskating to eyes, sxin and resolretory tract. ACUTE TOXICITY moder Eesiy toxis

Routes of ExposueE: tagustien, Inhatation, contact

TARCET ORGAMS: HoC deterninse

oxronte TOXTEity, Hat futernimid

Roures of Exposinet Not selirmined

TAREET ORGANSI not determined

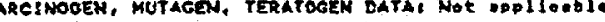

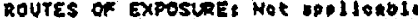

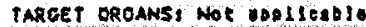

overexposuse: troltstian

\section{PRECAUTIONARY MEASURES}

Wish thar aushly ofter handins.

Avede coneriet wth oyos, akin and clothlite.

Do not bridthe enemtende.

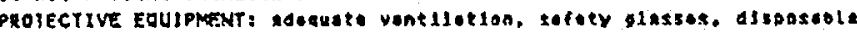

laves
VII. FIRST AID

tye un sxis conlacts Flush with olonty of wothe.

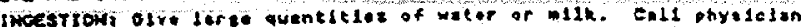
limedsetsly.

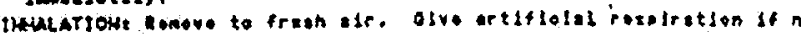
call phystolan.

VIII. SPILL AND DISPOSAL PROCEDURES

IH CASE OF SPILL OR RELEASES SEsop fato.

IX. TRAMSPORTATZOH DATA

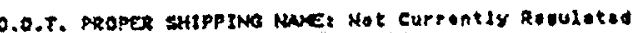

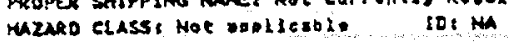

T.G.A.O. PROPER SH3PPtwo NAKE, Not CurroneIY Retuisted

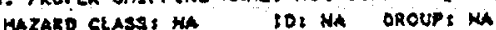

\section{$X$. AEFERENCES}

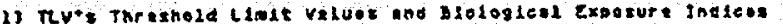

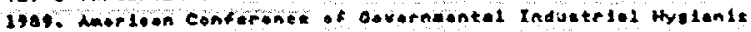

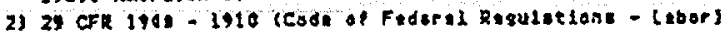

3) tn-housi intoration

4) Teonntelel Jussment

53 sax, N. trying. Dengerous Properties of industried totertale Hav vack: Von Nostrons Rointiajo co. IHac. 


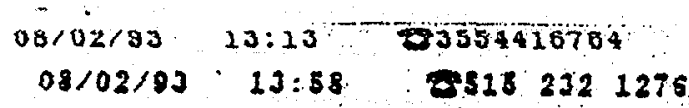

ent. No. 345
PGDP HXIS

HACH CO

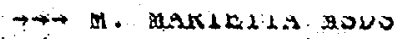

$\rightarrow \rightarrow$ RGDP INIS

436

\section{MATERIAL SAFETY DATA SHEET}

RECEIVED 2106021993

tossatmute Gonterst kefuletort iftolrs Buac.

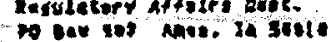
feres (x)-4tes

\section{MeH conpany}

00 .

MPS in seazo
Energendy relerbenk equen Howhtaln bolodo eer. (I0)s ses-5rik

\section{PNODUET IDEMIIICATION}

phever vanes seurah enthator solwess

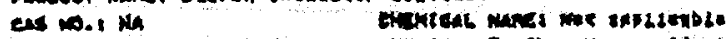

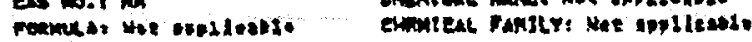

\section{ThenErIEMTs}

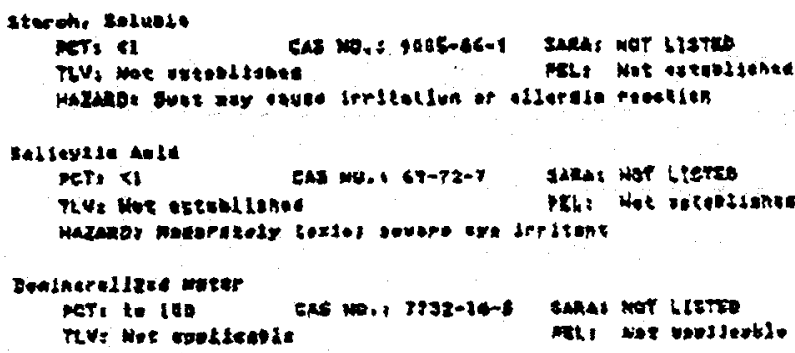

\section{PHYSIEAL DATA}

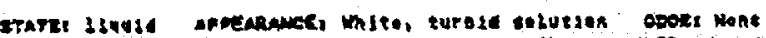

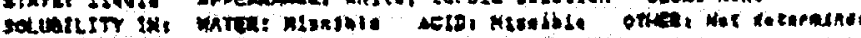

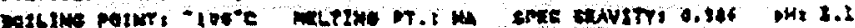

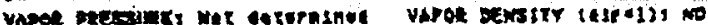

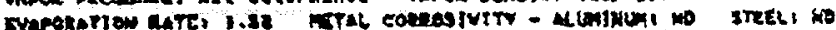
etaslletrs stubl.

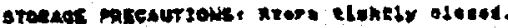

\section{IY. FIRE, ExpLosIan MZARD wo RELETIVITY DATA}

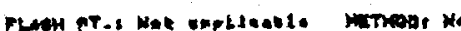

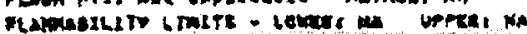

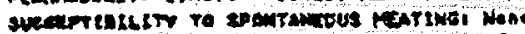

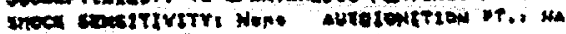

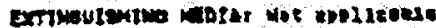

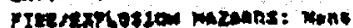

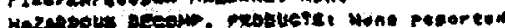

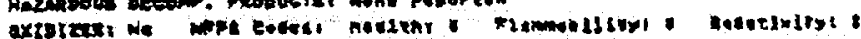
eovertsous to wotbi Nane

\section{HEALTH HATARD DATA}

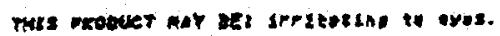

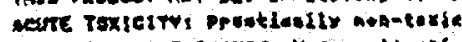

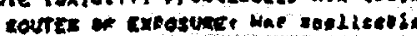

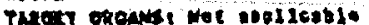

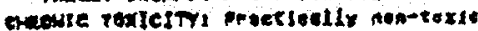

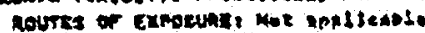

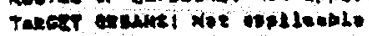

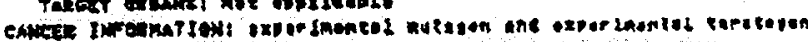

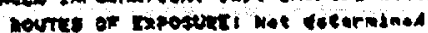

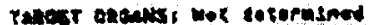

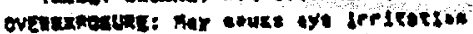

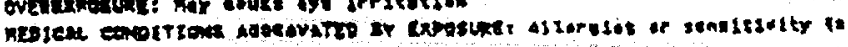
onllarile nolk.

\section{PAEEAUTROHAY MEASURTS}

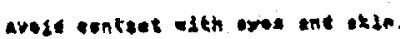

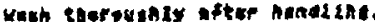

mertertve rsutemoti ist of sefo gosalok
UII. FIRST $\mathbf{A D}$

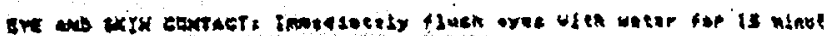

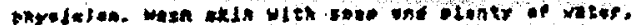

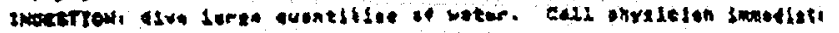

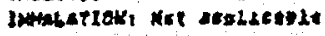

VIII. SPILL AND DISFOSAL PROCEDUKES

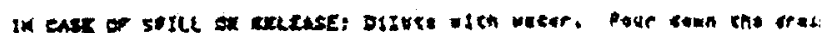
exeare wastr.

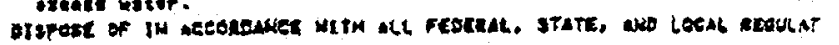

\section{Ix. Trakspontarton bata}

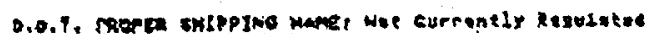

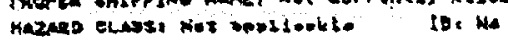

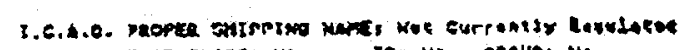

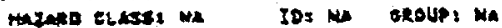

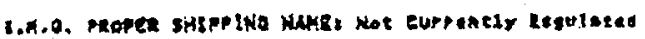

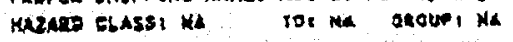

\section{REFTHEMCES}

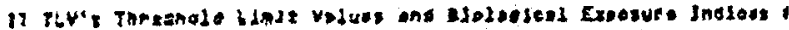

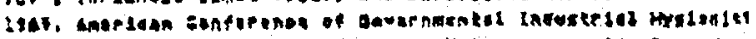

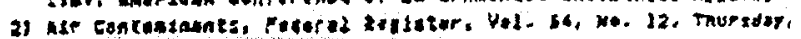

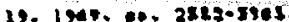

5s In-nesse infuratet ten

4) reohided gudgenent

i) Wonear inturpacian. 


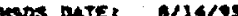
CHANGE No., 1475?
For hoblztenen, cantect: Resulatory APfalrs Deat.

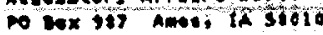
(8)9) 227-4224
HACH COAPANY

Po Box 907 WiES, IA 50010
Easersaney Talophone * Rooky Mauntin Palson Gts. (305) $223-5716$

\section{PHOOUCT IDENTIFICATIOM}

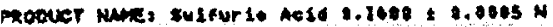

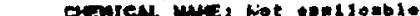

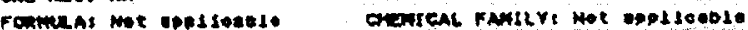

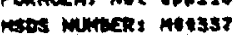

\section{IY. INENEDTEXTS}

sulfurte Aetd

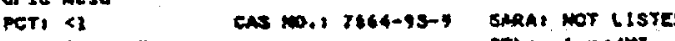

TiV, 1 masks Iancr L3etza

PELI: 1 noms

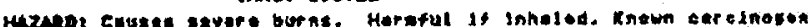

other oempenente, weah

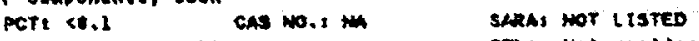

rutt Hot epl latble

mazaro: Not onsiseare

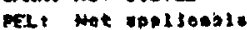

\section{Deselnaraldzed weter}

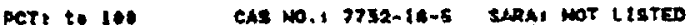

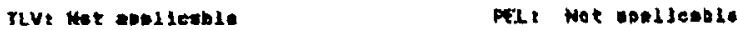

Hazamb. Mane

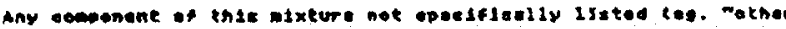

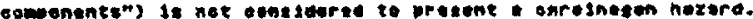

\section{PHYSICAl DATA}

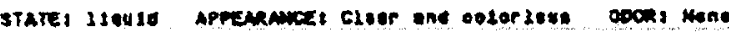

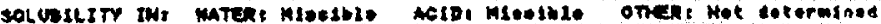

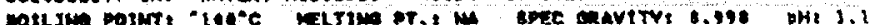

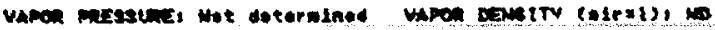

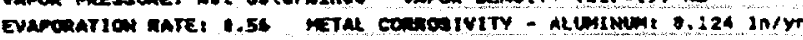

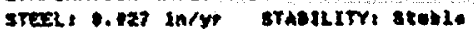

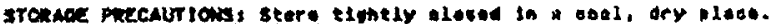

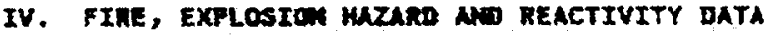

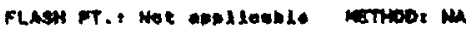

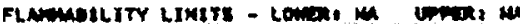

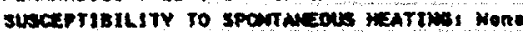

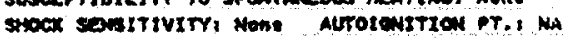

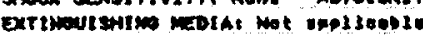

Freerexat oston menose Mone

Mazumbers occont. Probuersi Mone

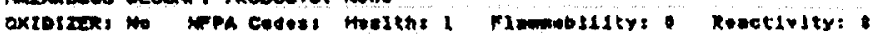

comoltions to avold: Extrene tomberstur we

\section{v. HEALTH MAZARD DATA}

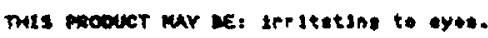

MCUTE roxfcitr, pretlouliy non-toxlo

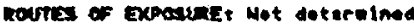

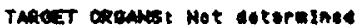

conowic rexiclive wot dotoralned

kourtes of exposunes Not deteralnas

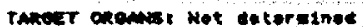

cucte infoumatrows in lnstedsent of thls mbxture is a known osrelnoen. corres of Exposuce inhalatien

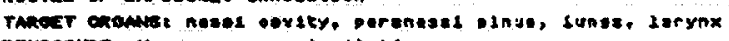

ovauxposine, Hoy ouven oye trritstion.

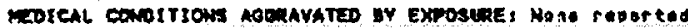

\section{vI. PnECAUTTONARY MEASURES}

\section{Avold tenteet with syes.}

wean thorecushis after nanesins.

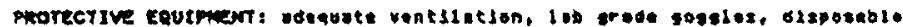
latox olowes, lat sodt

\section{FERST AID}

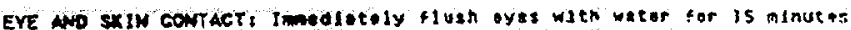
Cell whyslesan.

Imestion: olve iarse quantiting of veter. Call physielen ingedintely

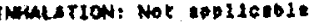

\section{YIII. SPILL AMO DISPOSAL PROCEDURES}

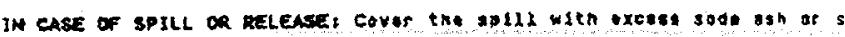

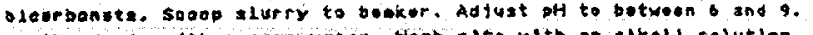
to the droin wien exesx wetor. Wesh slte with in skell solution.

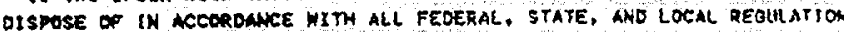

\section{TRAHSTORTATIOA DATA}

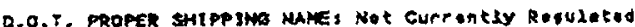
HaxakD CLASS! MA IDS MA GROUP: NA

I.E.A.0. PROFER SHIPPINo Mare? Not Cursently Roguleted

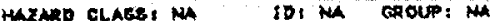

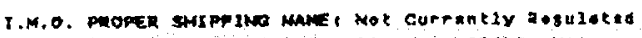
inzand Classi in IO: HA DRoup: MA

\section{$X$. REFEDERCES}

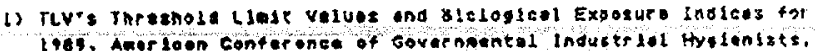

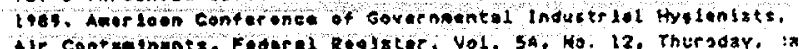

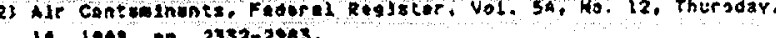
14. 1949, 2332-2983.

3) Techndeet Juekeme

4) in-houxt information

5) [ahc Mowograbn on the Evaluation of Carcinosande alsk to Huapns, kealth organization (volumb 54) 1932, froncs.

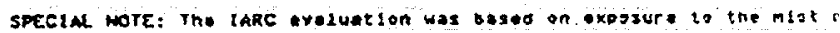

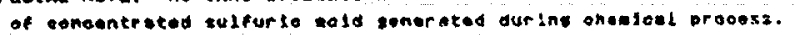


car. no.

MSDS DATE: 1/12J4S

CHatioc 10.: \$74B
MATERIAL SAEETY DATA SHEET

For desitetenes, Contect: Rerulatery hefelre Dept.

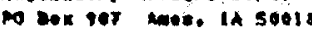
(6911) $227-4224$

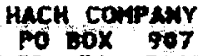

Emereoney Tolephene Rowky mountuin potson ctr. (3)3) $623-5716$

\section{PNow InETTIFICATIO}

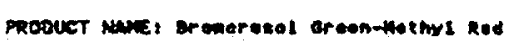

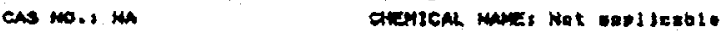

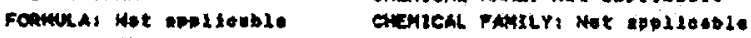

mas momeri moets?

\section{THoneorents}

Potestivin chloride

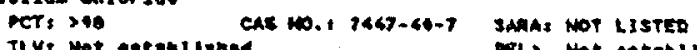

Tive not tekendished

HazARDi mey owGre eye Irrstetion

Pel: Not ostablisinod

other opeponents, wesh

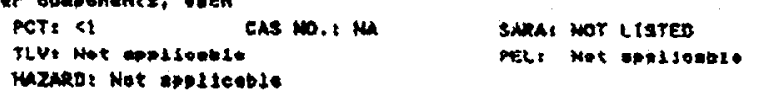

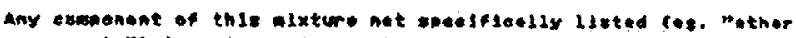

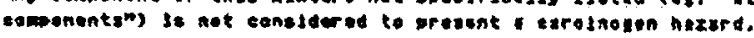

\section{PHYSTCAL DATA}

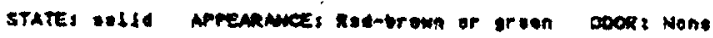

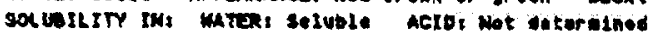

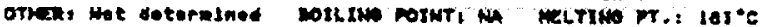

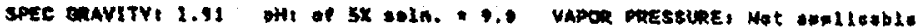

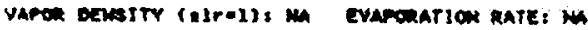

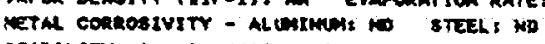

sTABltity, ses conditsons to dvold

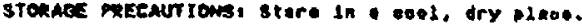

\section{FIRE, EXPLOSION MRIRD AND REACTEYITY DATA}

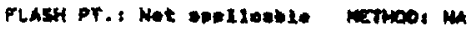

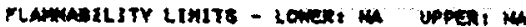

susceptiallitr to spowratzous heattio, None

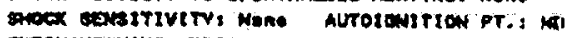

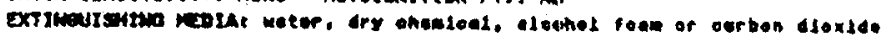

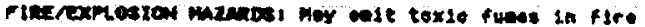

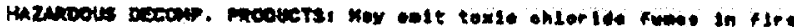

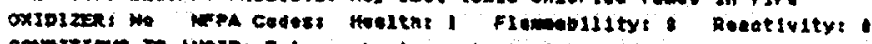

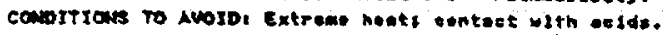

\section{HEALTH HAZARD DATA}

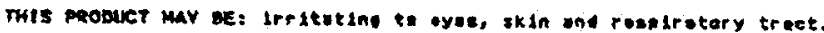

ACUTE TOXieiry! Hodorately teis

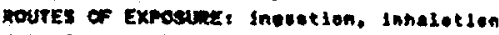

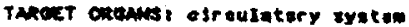

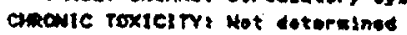

moutes or exposine t wot dotarninad

TAngeT conous? Not dotaralned

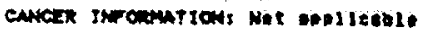

Routes of Exposinti Not ippllowite

Takeet onans, Mat apolleshle.

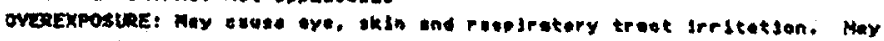

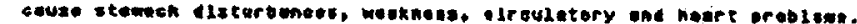

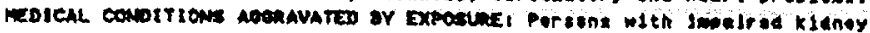

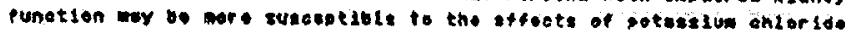

\section{PRECAUTTOMARY MEASURES}

Ayose contwot wsth oyes and skin.

De not orenth. dust.

wish thoroushly ifter nondlins.

xato may erom ectde.

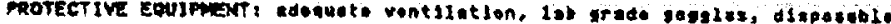
Lotex gleves

\section{FInST AID}

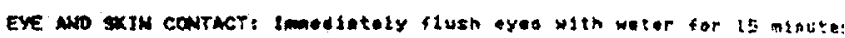
psyssolan. Fiuan oth in with plonty of wotar.

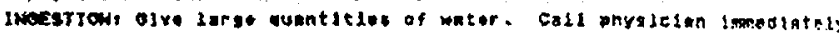
THalations gemove to tresh alr.

\section{SPILL ANO DISPOSAL PROCEMURES}

IN CASE OF SPILL DR RELEASE, sweep up pawdor. Avold trouthshg mater Dsoselue in witer. Flush bown the orain wlth oxoust water.

Dispose of iN ACCORDMUE WITH ALL FEDERAL, STATE. AHD LOCAL REESLAT:O

\section{TRAMSPRTATION DATA}

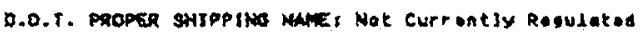
Hazane CLAS3, in 10 : MA GROUP: MA

T.E.A.O. PRoper SHIPPINO Mure: Not Curronty Regulsted MAZMAD CLARS, WA TD: NA GRCUP: WA

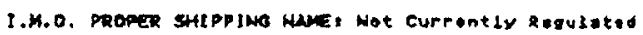

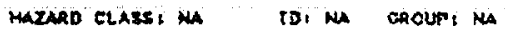

\section{$X$. REFEREKCES}

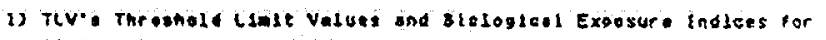

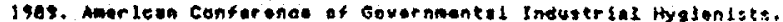

2) Alr conteminente, Foctoral Rasistog, Vol. 54, No. 12, Thurstay, . 14. 3469. $3332-2463$.

3) in-house inforestion

6) Pochntral Judgeunt 


\section{MATERIAL SAFETY DATA SHEET}

L. TRADENAMES: DISSOLVED OXYGENCHEMULS, ULR CHEMatoo and Vacu-Vialso

CATALOQ NOS: R-7501, R-7501U, R.7501V. R-7511, R-7518, R-7518U, R.7540, R-7540G. A-754ON, F-7SLOU, R-7540V, A.7553, K.7580U, K.7599 and K-7599Y

OESCRIPTION: Rasgan ampoutes for the determinaton of dlssolvad sxygen in water. Each CHEMerm conteins epproximaleiy $0.50 \mathrm{~mL}$ of liquid reagent soaled under vacuUm. Each ULA CHEMolm contains aporoximatery $1 \mathrm{~mL}$ of frouid reagent asaled under vacuum. Each Vacu-viatm contains approxinatily $2 \mathrm{~mL}$ of liculd reagant seaied under vecuum.

NFPA PATINGS: HEALTH: 1 FLAMMABILTY: O REACTIVITY: O

\section{COMPONENTS}

COMPONENT: Dialmiene Glycal

CASNO.: $111-46-6$ PERCENT: 25.0

COMPONENT: Coionizad Waler

CAS NO.: 7732.18 .5 PERCENT: $>74.0$

COMPONENT: Cthet componenta

CAS NO: NA PEACENT: $<1.0$

Any component of this mixture not spectifally fisled (0.9. "other compo fants") is not considered to present a carcinogen hazard.

\section{PHYSICAL DATA}

STATE: UQUId APPEARANCE: Coloriess lo pale yellow ODOA: None SOLUBILTY IN WATEA: Miscible PH: 11.5 GOILNG POINT: $150^{\circ} \mathrm{C}$ MELTING POINT: $-5.0^{\circ} \mathrm{C}$ VAPOA FRESSURE: NA SPEEIFIC GRAVITY $(H Q=1): 1.03$ VAPOA DENSITY \{AIA - 1\}: N/A

\section{FIRE AND EXPLOSION DATA}

FLASH POINT: NA AUTOIGNITION FOINT: NA FLAMMABILTY LIMITS: UPPER: N/A LOWER: NIA EXTINGUISHING MEDIA: ON chemkal, catoon dioxida, water spray or toam

\section{REACTIVITY DATA}

HAZARDOUS OECOMPOSITION PROOUCTS: NONO

\section{HEALTH HAZARD DATA}

OSHA \& ACGIH THRESHOLO UMIT VALUE: NONB ERtallshed ACUTE TOXICITY: Imtation

CHRONIC TOXICITY: Imitation

CARCINOGEN STATUS: NON

MEDICAL CONOITIONS AGQRAVATED BY EXPOSURE: MaY CaUde itriation to oyes, skin and mutous mambranes.

\section{FIRST AIO}

EVE AND SKIN CONTACT. Immodialaly llush eyes and okin with watar lor 15 minutoo.

INGESTION: Sook mecleal attention.

WHALATION: Removo IndWOWal to trean air.

\section{PRECAUTIONS}

It thie proctust is uned as diroclad, the uoer will not come in conbet wh or bo exposed to any of its enemical components.

Wash ihoroughly atter tandling.

Avoid contser wilh eyse.

FPAGILE: Llquid in giass. Handlo with care.

PROTECTIVE EOUIPMENT: SalatY qlaseas

\section{SPILL AND DISPOSAL PROCEDURES}

Take un with abaorbent materiat. Place in amall sentaners for disposal. Dispose of in accordence with all Foderal, stale and Local Regutations.

\section{$x$ STORAGE CONDITIONS}

Product should be thored in the dark and at room temperalure; howevor, temperatures up to $120^{\circ}$ F or oven balow trouzing will nol nomally aftect reagen pertormance.

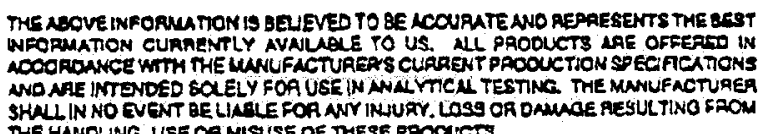

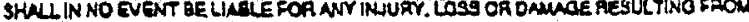
THE HANOLNG, USE OA NISUSE OF THESE PAOCUCTS.

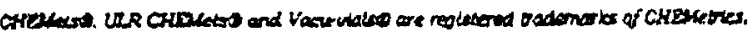
ine 


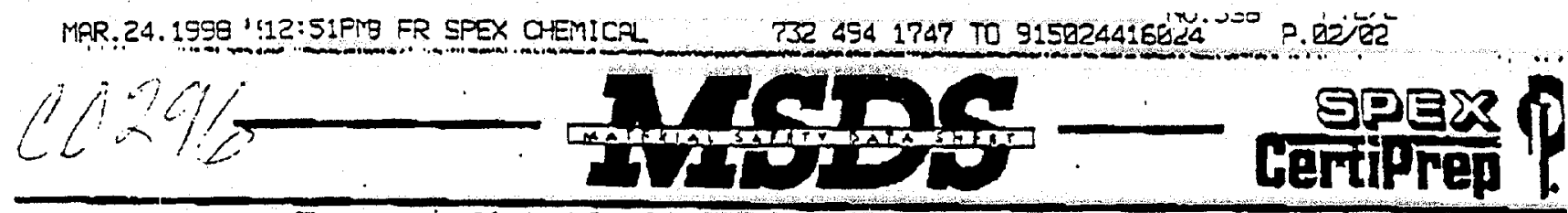

Emergency. Phone Number (24 hours) CHEMTREC: $1.800-424-9300$

\section{SECTIONI}

NAME: Custom Anion Standard - Ion. Chromasography' Solution Standard: 1,000 ppm of Bromide (from Sodium Bromide) in water. CHEMICAI FAMILY: Dilure Salt Solurion. COMMON NAME OR SYNONYMS: None. SPEX CERTIPREP CATALOG NUMBER: AS.BR9.2Y or ASBR9-2X

\section{PRECAUTIONARY DATA}

Sirce $B_{r}$ - is in solution at less than $10,000 \mathrm{ppm}$ (or 1\%), it is not required to be reporred as Healtb Hazard's according to OSFA.

This material is not known to contain any chemical listed as a careinogen or suspected carcinogen at a concentration greater than $0.1 \%$ by OSFA, IARC, or NTP.

Use careful laborarory rechoigue when working with any chersical whecher it is considered a Health Hazard or not. Avoid inbalation, ingestion, and contace with the skin and eyes. Use prorective gloves, glasses and clothing.

WHMIS Classification (Canada): Nor regulated

$\frac{\text { SECTIONII }}{\text { PHYSICAI DATA }}$

SPECIFIC GRAVITY: Approximately 1.

SOLUBIIITY IN WATER: Soluble in wares.

APPEARANCE AND ODOR: Transparens solution withour an odor.

\section{NOTICE}

SPEX CERTIPREP, INC. ASSUMES NO RESPONSIBIITY AND MAKES NO

WARRANTIES, EXPRESS OR IMPIIED, AS TO THE ACCURACY OR COMPLETENESS OF THE DATA CONTAINED HEREIN. INDICATED SAFETY MEASURES MAY NOT REFLECT ALL A PPROPRIATE SAFETY MEASURES. SREX CERTIPREP, INC. ASSUMES THIA I ONLY QUAIIFIED INDIVDUAIS, TRANNED AND FAMIIIAR WITH PROCEDURES SUTTABIE TO IHIS PROCEDURE, WIIL
HANDIE IHIS PRODUCT.

Date: March 20, 1997

Authorized Signazure

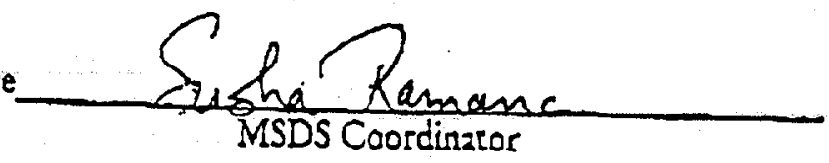




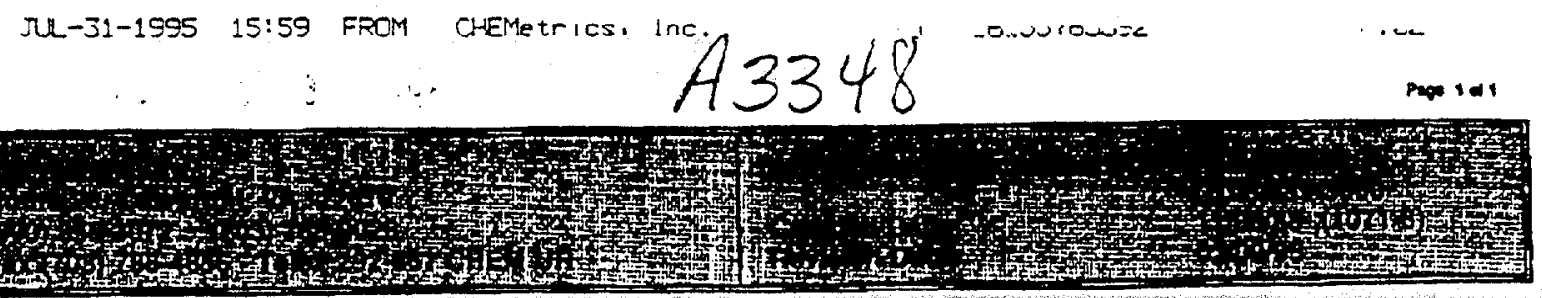

\section{MATERIAL SAFETY DATA SHEET}

\section{TRADE NAMES: DISSOLVED OXYGEN CHEMts and Vicu-rialso}

CNTALoe nos: K-7502, K-7510, R-7503, R-7512, R.7512U and R-7513

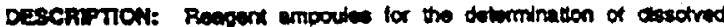

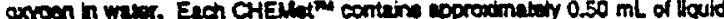

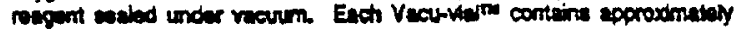
$2 \mathrm{~mL}$ of lieuld respent sediod under vacum.

NFPA RATHYS: HEALTH: I FUMAABLTT: O REACTINTY: O

\section{a. COMPONENTS}

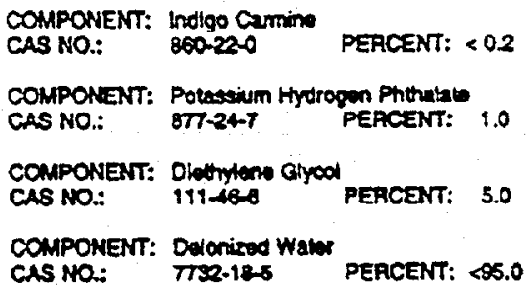

COMPONENT: Other components

CAS NO: NA PEACENT: $<1.0$

Am component of this mixure not soctically listed (o.g. "other componente 7 is not conaidered to provent e carcinogen hazard.

\section{IIL PHYSTCAL DATA}

STATE: UQW' APPEARANCE: Yollow ODOR: None SOUBUTY W WATER: Compini pH: A

BOLING PONT: $1100 \mathrm{C}$ NELTINO PONT: $.10 \mathrm{C}$

YAFOR PAESSURE: NA SPECIFX GRAVTY: 1.01

YAPCA DENSTY: NA

\section{GRE AND EXPLOSION DATA}

FLASH PONT: NA AUTOGNITON PONT: NA

FUAAMBIUTY UMTIS: UPPEA: NA LOWER: NA

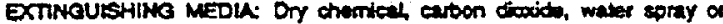
$\operatorname{lom}$

\section{REACTIVIY DATA}

HAZARDOUS DECOMPOSTIION PROOUCTS: NONO

\section{HEALTH HAZARD DATA}

OSH \& ACSH THRESHOD LMT VMUE: Hon wtabtiand ACUTE TOXICTY: bitution

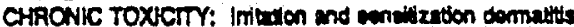
CARCNOOEN STATUS: Non

MEDKAL CONDITONO MCOARAVATED BY EXPOSURE: MaY CQUS

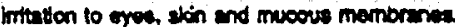

Vi. FIRST AID

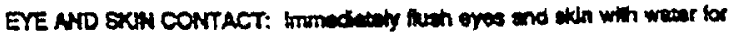
16 minutes.

Wesstion: Seck madicel attention.

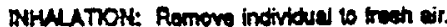

\section{VIIL PRECAUTIONS}

It the product is used as olrected, the user will not coms in contact with of be expoesd to any of the churiticel componeme.

Wean trorougty ther tranding.

Aveld contace wis oyes.

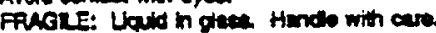

PROTECTIVE EOUIFMENT: SaleY diasen

\section{SPIL ANO DISPOSAL PROCEDURES}

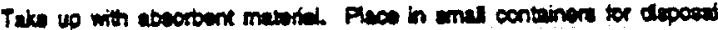

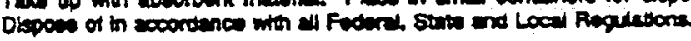

\section{$X$ STORAGE CONOITOHS}

Product thould be wand in the datk and at room tomponsure howner

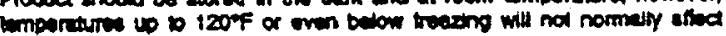
megent purtomance.

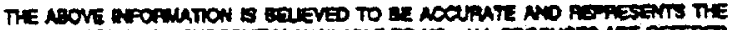

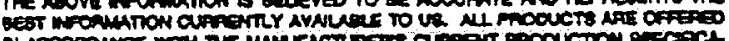

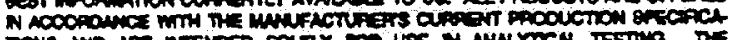

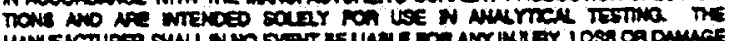

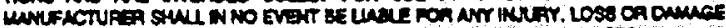

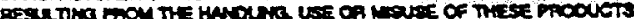

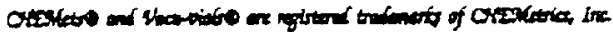




\section{Material Safety Data Sheet}

\section{CHEMICAL PRODUCT AND COMPANY IDENTIFICATION}

Product Namc: Ferrous Iron Reagent Powder Pillows

Catalog Number: 1037

Hach Company

P.O.Box 389

Loveland, CO USA 80539

(970) 669-3050
Emergency Telephone Numbers:

(Medical and Transportation)

(303) 623-5716 24 Hour Service

(515)232-2533 8am-4pm CST

MSDS Number: M00024

Chemical Name: Not applicable

CAS No.: Nol applicable

Chemical Formula: Not applicable

Chemical Family: Not applicable

Hazard: May cause irritation.

Date of MSDS Preparation:

Day: 30

Month: August

Year: 1999

\section{COMPOSITION/INFORMATION ON INGREDIENTS}

1, 10-Phenanthroline

CAS No.: 5144-89.8

TSCA CAS Number: $66-71-7$

Percent Range: $1.0-10.0$

Percent Range Units: weight/ weight

LDS0: Oral Rat $L D_{50}=132 \mathrm{mg} / \mathrm{kg}$

LC50: None reported

TLV: Not established

PEL: Not established

Hazard: May cause irritation.

\section{Sodium Bicarbonate}

CAS No.: 144-55.8

TSCA CAS Number: $144-55.8$

Percent Range: 90.0 - 100.0

Percent Range Units: weight/weight

LDSO: Oral rat $L D 50=4220 \mathrm{mg} / \mathrm{kg}$

LC50: None reported

$T L Y:$ Not established

PEL: Not established

Hazard: May cause irritation.

\section{HAZARDS IDENTIFICATION}




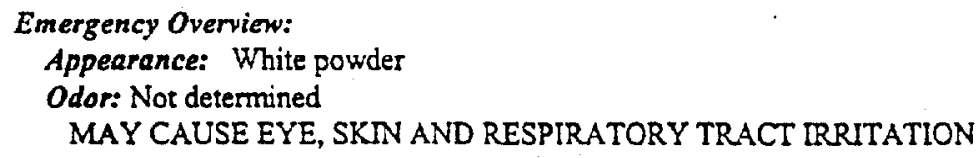

This product does NOT contain any IARC listed chemicals.

This product does NOT contain any NTP listed chemicals.

Additional Cancer / Reproductive Toxicity Information: None reported Toxicologically Synergistic Products: None reported

\section{FIRST AID}

Eye Contact: Immediately flush eyes with water for 15 minutes. Call physician. Skin Contact (First Aid): Wash skin with soap and plenty of water. Call physician if irritation develops. Ingestion (First Aid): Give large quantities of water. Call physician immediately. Inhalation: Remove to fresh air.

\section{FIRE FIGHTING MEASURES}

Flammable Properties: Does not bum, but may melt in a fire, releasing toxic fumes.

Flash Point: Not applicable 
Method: Not applicable

Flammability Limits:

Lower Explosion Limits: Not applicable

Upper Explosion Limits: Not applicabic

Autoignition Temperature: Not applicable

Hazardous Combustion Products: Toxic fumes of: sodium monoxide nitrogen oxides. carbon monoxide. carbon dioxide.

Fire / Explosion Hazards: None reported

Static Discharge: None reported.

Mechanical Impact: None reported

Extinguishing Media: Water. Carbon dioxide Dry chemical.

Fire Fighting Instruction: As in any fire, wear self-contained breathing apparatus pressure-demand and full protective gear.

\section{ACCIDENTAL RELEASE MEASURES}

\section{Spill Response Notice:}

Only persons properly qualified to respond to an emergency involving hazardous substances may respond to a spill according to federal regulations (OSHA 29 CFR 1910.120(a)(v)) and per your company's emergency response plan and guidelines/procedures. See Section 13, Special Instructions for disposal assistance. Containment Technique: Stop spilled material from being released to the environment.

Clean-up Technique: Scoop up spilled material into a large beaker and dissolve with water. Flush the spilled material to the drain with a large excess of water. Decontaminate the area of the spill with a weak acid solution. Evacuation Procedure: Evacuate as needed to periom spill clean-up. If conditions warrant, increase the size of the evacuation.

Special Instructions (for accidental release): Not applicable

304 EHS RQ (40 CFR 355): Not applicable

D.O.T. Emergency Response Guide Number: None

\section{HANDLING / STORAGE}

Handling: Avoid contzet with eyes skin Do not breathe dust. Wash thoroughly after handling. Maintain general industrial hygiene practices when using this product.

Storage: Keep container tightly closed when not in use. Protect from: moisture oxidizers

Flammability Class: Not applicable

\section{EXPOSURE CONTROLS / PROTECTIVE EQUIPMENT}

Engineering Controls: Have an eyewash station nearby. Maintain general industrial hygiene practices when using this product.

\section{Personal Protective Equipment:}

Eye Protection: safety glasses with top and side shields

Skin Protection: disposable latex gloves

Inhalation Protection: adequate ventilation

Precautionary Measures: Avoid contact with: eyes skin Do not breathe: dust Wash thoroughly after handling. Keep away from: oxidizers

TLV: Not established

$P E L:$ Not established

\section{PHYSICAL / CHEMICAL PROPERTIES}


Warld Headquarters

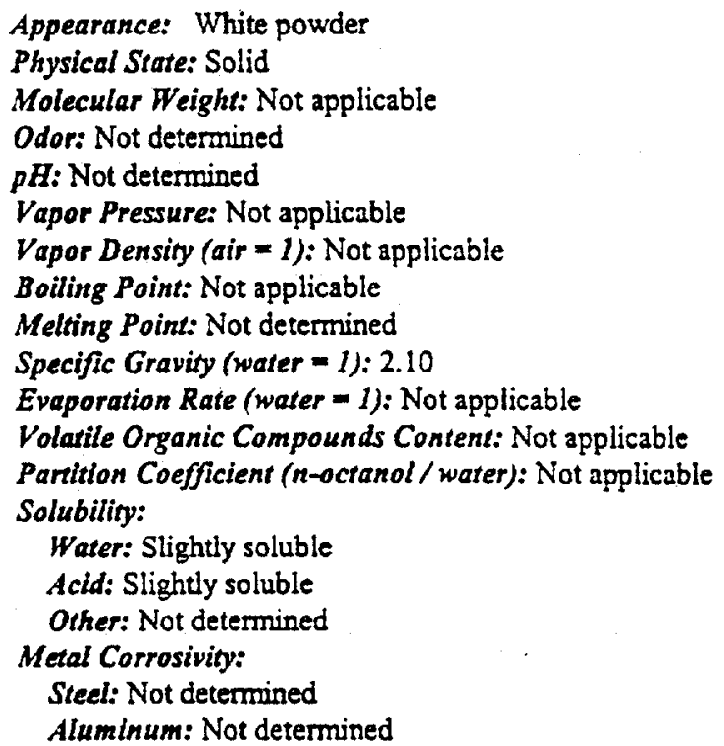

\section{STABILITY/REACTIVITY}

Chemical Stability: Stable when stored under proper conditions.

Conditions to Aroid: Excess moisture Heating to decomposition.

Reactivity/Incompatibility: Incompatible with: oxidizers

Hazardous Decomposition: Toxic fumes of: nitrogen oxides sodium oxides carbon monoxide carbon dioxide

Hazardous Polymerization: Will not occur.

\section{TOXICOLOGICAL INFORMATION}

Product Toxicological Data:

LDSO: None reported

LCSO: None reported

Dermal Toxicity Data: None reported

Skin and Eye Irritation Data: Sodium Bicarbonate: Eye - rabbit - $100 \mathrm{mg} / 30$ seconds - MILD; Skin -

Human - $30 \mathrm{mg} / 3$ days intermittent - MILD

Mutarion Data: None reported

Reproductive Effects Data: None reported

Ingredient Toxicological Data: Sodium Bicarbonate: Oral rat $\mathrm{LD}_{50}=4220 \mathrm{mg} / \mathrm{kg} ; \quad 1,10$-Phenanthroline: Oral rat $L D_{50}=132 \mathrm{mg} / \mathrm{kg}$

\section{ECOLOGICAL INFORMATION}

Product Ecological Information: -

No ecological data available for this product.

Ingredlent Ecological Information: -- 
No ecological data available for the ingredients of this product.

\section{DISPOSAL CONSIDERATIONS}

EPA Waste DD Number: None

Special Instructions (Disposaf): Dilute material with excess water making a weaker than $5 \%$ solution. Open cold water tap completely, slowly pour the material to the drain.

Empty Containers: Rinse three times with an appropriate solvent. Dispose of empty container as normal trash.

NOTICE (Dlsposal): These disposal guidelines are based on federal regulations and may be superseded by more stringent state or local requirements. Please consuit your local environmental regulators for more information.

\section{TRANSPORT INFORMATION}

D.O.T.:

D.O.T. Proper Shipping Name: Not Currently Regulated

-

DOT Hazard Class: NA

DOT Subsidiary Risk: NA

DOT ID Number: NA

DOT Packing Group: NA

I.C.A.O.:

I.C.A.O. Proper Shipping Name: Not Currently Regulated

$-$

ICAO Hazard Class: NA

ICAO Subsidiary Risk: NA

ICAO ID Number: NA

ICAO Packing Group: NA

I.M.O.:

I.M.O. Proper Shipping Name: Not Currently Regulated

$-$

I.M.O. Hazard Class: NA

I.M.O. Subsidiary Risk: NA

I.M.O. ID Number: NA

I.M.O. Packing Group: NA

\section{REGULATORY INFORMATION}

\section{U.S. Federal Regulations:}

O.S.H.A.: This product meets the criteria for a hazarcous substance as defined in the Hazard

Communication Standard. (29 CFR 1910.1200)

E.P.A.:

S.A.R.A. Title III Section 311/312 Categorization (40 CFR 370): Immediate (Acute) Health Hazard S.A.R.A. Title III Section 313 (40 CFR 372): This product does NOT contain any chemical subject to the reporting requirements of Section 313 of Title III of SARA.

$-$

302 (EHS) TPQ (40 CFR 355): Not applicable 304 CERCLA RQ (\$O CFR 302.4): Not applicable $30 \$$ EHS RQ ( 40 CFR 355): Not applicable 
Clean Water Act ( 40 CFR 116.4): Not applicable

RCRA: Contains no RCRA regulated substances.

C.P.S.C.: Not applicable

State Regulations:

Callformia Prop. 65: No Prop. 65 listed chemicals are present in this product. Identification of Prop. 65 Ingredient(s): None

Trade Secret Registry: Not applicable

National Inventories:

U.S. Inventory Status: All ingredients in this product are listed on the TSCA 8(b) Inventory (40 CFR 710). TSCA CAS Number: Not applicable

\section{OTHER INFORMATION}

Intended Use: Iron determination

References: TLV's Threshold Limit Values and Biological Exposure Indices for 1992-1993. American Conference of Governmental Industrial Hygienists, 1992. Air Contaminants, Federal Register, Vol. 54, No. 12. Thursday, January 19, 1989. pp. 2332-2983. 29 CFR 1900 - 1910 (Code of Federal Regulations - Labor). Inhouse information. Technical Judgment. Fire Protection Guide on Hazarcous Materials, 10th Ed. Quincy, MA: National Fire Protection Fire Protection Guide on Hazardous Materials, 10th Ed. Quincy, MA: National Fire Protection Association, 1991.

Legend:

$\begin{array}{ll}\text { NA - Not Applicable } & w / w-\text { weight/weight } \\ \text { ND - Not Determined } & \text { w/v - weight/volume } \\ \text { NV-Not Available } & \text { w/v-volume/volume }\end{array}$

USER RESPONSIBILITY: Each user should read and understand this information and incorporate it in individual site safety programs in accordance with applicable hazard communication standards and regulations.

THE INFORMATION CONTAINED HEREIN IS BASED ON DATA CONSIDERED TO BE ACCURATE. HOWEVER, NO WARRAVTY IS EXPRESSED OR IMPLIED REGARDIVG THE ACCURACY OF THESE DATA OR THE RESULTS TO BE OBTAINED FROM THE USE THEREOF.

\section{HACH COMPANY O1999}




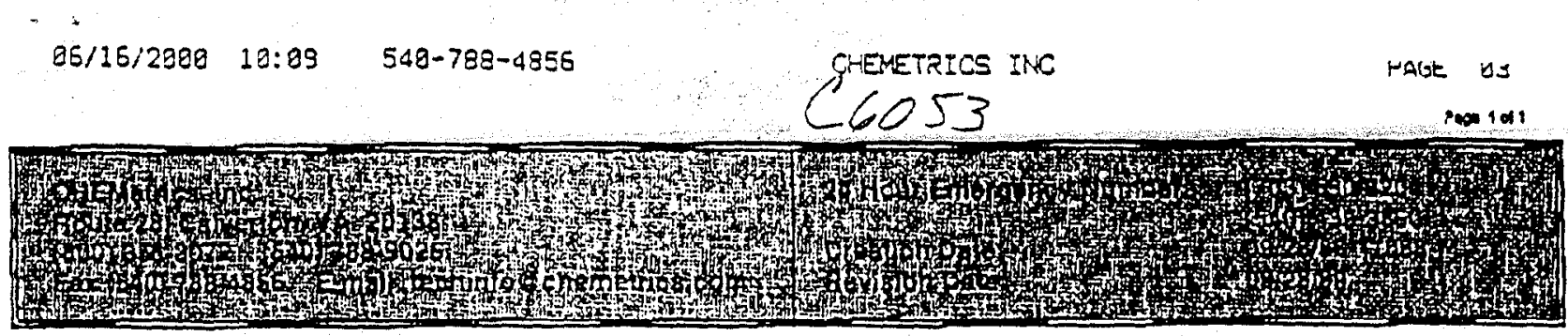

\section{MATERIAL SAFETY DATA SHEET}

\section{CHEMICAL IDENTIFICATION}

TAAOE NAME: SULIDE ACTIVATOR SOLUTION

CATALOGNO:: A.9500

QESCAIPTION: An ecoseany solution used in conjunction with reagent umpouter in the delermination of sutslda in water. Each bonta contring scproximately $10 \mathrm{~mL}$ of acceccory solution

NFPA PATINGS: HEALTH: 9 FLAMMABIUTT: 0 REACTIVITY: 0

\section{COMPOSITIONMNFORKATION ON INGAEDIENTS}

COMPONENT: Fame Chlorde, Hexampdrete

CAS NO: $\quad 10025-77-1$ PERCENT: 20.0

COMPONENT: Hydrechlorle Adr

CAS NO:: T647.01.0 PEACENT: $>70.0$

\section{HAZARDS IDENTIFICATION}

OANGERt Combtve Caused oyo and skin bums. Cauges digestive and "espiratory tract bums

TARQET ORGANS: None

ACUTE TOXICTY: Irtration and bum

CARONIC TOXICIT\%: Imiation and ans beation demalito

MEDICAL CONDITIONS AGGAAVATED BY EXPOSURE: MaY CaUHa tritation to oyes, skin and mucous membranoc. May eaws cermatis. conjunctivitis, oums and toeth erosion.

\section{FIRST AID MEASUAES}

EYE AND SXIN CONTACT: Immockathly / ush oysa and skin when wacor for is minuta.

INGESTION: It conscious, give 2.4 cups of milk or water. Seak modlcal altention.

INHALATION: Remove Individus to treeh alr.

\section{FIRE FGGTING MEASURES}

FLASH POINT: NA AUTOIGNTION POINT: NA FLAMMABILTY LIMITS: UPPER: NA LOWER: NA EXTINGUISHINQ MEOLA: ONy chemical, carbon dioxids or alconol toam

\section{ACCIOENTAL RELEASE MEASURES}

Take up with absorbent malerial. Place in small centsinen tor olsoosal. Vanbilato area and wach splil stis shet material pick up is complete.

\section{HANDLING AND STORAGE}

If thls product is used as dineted, the user will not come in contact whth or bo exposed to any of its chemical components. Wach thoroughty attup hanetting A void conlect with oyas.

Product should be slorsd in the dark and at room lemperalum; however. lemperatures up to $120^{\circ} \mathrm{F}$ of oven below freezing will not nomally affect reagent portomenco.

\section{EXPOSURE CONTROLSI PERSONAL PROTECTION}

OSHA L ACGIH THAESHOLD LIMT VALUE: 5 pOM Hydrochlaric AdO PROTECTIVE EOUIPMENT: SAfory glasess

\section{PHYSICAL AND CHEMICAL PROPEATES}

STATE: Lquid APPEARANCE: Brownish gold ODOR: Pungent SOUBIUTY IN WATER: WSGDio PH: $<1$

BOIUNO POINT: $196^{\circ} \mathrm{C}$ MEI TNNO FONT: NA VAPOR FRESSIAE: NA SPECIFIC GRAVITY: 1.2 VAPOR OENSITY: 13

\section{$x$ STABILITY AND REACTIVTY}

HAZARDOUS DECOMPOSTION PAODUCTS: Enits tode lumes. Heating raleasos Increasing anounta of mydrogen chloride.

\section{TOXICOLOGICAL NFORMATION}

Carchogen Searus (tyydroshloric Acld): ACOIH: (A2) - Bupoucled human cartnogen NTP: Suspect cardinogen OSHA: Possble selece carcinogan IAAC: Group 3 caranogen

\section{ECOLOGICAL INFOAMATION}

Envtronmental Fate: Sthatance will neuralize rod carbonatobased componente.

\section{DISPOSAL CONSIDERATIONS}

Dlspose of in a mannar consistont with Foderal, State and Losal Regulatione, Not listec as a matertal bamed from land sleposel acoerding to RCRA

\section{XN. TRANSPOAT INFOAMATION}

US DOT: 49 CFA, 173.4 Exwoonons For Small Cuantities

IATA: Denoprous Goods in Excepted Ounntition Hazard Clast: 8 UN Number. UN 1789

\section{XY. REGULATORY INFORMATION}

EUROPEAN INFORMATION:

Rlsk Phrases:

Corropive. Causes Bums. Intrating to Reepiratory Systam Salory Phrases:

in case of contact with eyos, inse limmediatly with plenty of water and seok medienl corles.

In casa of accident or if you toal unwoli seek medical actrica inmedlately (show labol whara poseltata).

U.S. INFOAMATION:

Al chemicel comporents of his product are liatad on the TSCA Imvontery. This metertel contalns mydroctiorie acid milen is sublect to the roporting requiraments of Section 313 of SARA Thl iti and 10 CFR 373

\section{XVL. OTHER INFORMATION}

THE AQOVE WEORMATIONIS BEUEVEO TO QE ACCUAATE WNO ROPAESENTS THE BEST INFOPNATON CUAAENTY AVALABLE TO US. $M L$ PAOOUCTS APE OFERED IM LCCORONCE WTMM THE WUNUFACTUFERS CUARONT PRCOUCTON SP GOFCATIONS AND AAE INTENDEO COLELY FOA USE IN AYA YTICAL TESTINO. TEE MAWUFACTUAEA

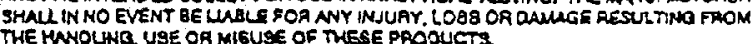




\section{MATERIAL SAFETY DATA SHEET}

\section{CHEMICAL IDENTIFICATION}

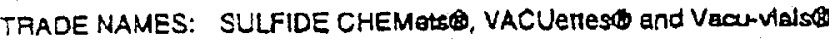

CATALOONOS.: R-9503, R-9510. R-9510A, R-3510日, R-9510C, R-9510D, R.9510 and $\mathrm{A} .9523$

DESCRIPTION: Resgent ampoules for the detenminalion of eulfide in water. Eech CHEMerm and VACUollen contalns approximately $0.50 \mathrm{~mL}$ of llquld reagent soaied undor vacusm. The R. 9503 Vacu-vialg contain approximately $2 \mathrm{~mL}$ of liquid reagent sealed undervacuum. The A-9523 Vacu-vialse contain epproxmately $4.5 \mathrm{~mL}$ of liquid reagens selaed under vacuum.

NFPA RATINGS: HEALTH: 3 FLAMMABILTY: O REACTIVITY: 0

\section{COMPOSITIONINFORMATION ON INGREDIENTS}

COMPONENT: N,N,-Olmeihyl-p-phemylene dlamine oxalate

CAS NO: $\quad$ E2T78.12-5 PERCENT: $<1.0$

COMPONENT: isOpropyl A/cohol

CASNO:: A7-E3-0 PERCENT: 2.0

COMPONENT: Hydrochlartc Add

CAS NO:: 7847.01.0 PERCENT: 25.0

COMPONENT: Deionized Water

CAS NO.: $7732-18-5$ PERCENT: $>70.0$

\section{HAZARDS IDENTIFICATION}

OANGERI Cortosive. Causes oyo and skin buma. Causes dilgesitue and respiratory tract burns.

TAAGET ORGANS: None

ACUTE TOXICITY: Irritation and bums

CHRONIC TOXICITY: Irftation and sensilization dormatlts

MEDICAL CONDITIONS AGGRAVATED gY EXPOSURE: May cause irritation to eyes, sXIn and mucous memoranes. May cause dermatits, corjunctuitts, bums and testh erosion.

\section{FIRST AID MEASURES}

EYE AND SKIN CONTACT: Immedlatehy flueh eyes and sxln with watep for 16 minuta:

INGESTION: If consctous. give 2.4 cups of milk or water. Soek medical attantion.

INHALATION: Remove indivdual to tresh air.

\section{FIRE FIGHTING MEASURES}

FLASH POINT: N/A AUTOIQNITION POINT: N/A

FLAMMABILITY LIMITS: UPPER: N/A LOWER: N/A

EXTINGUISHING MEOIA: ONy chemical, carbon dioxide or alcohol foam

\section{ACCIDENTAL RELEASE MEASURES}

Take up with absorbent material. Place In smail containert lor disposal. Ventilate ares and wash splil ste aftor matorial pick up is complete.

\section{HANDLING AND STORAGE}

If this produet ls used as directed, the user will not came in contaot with or be exposod to any of ite chemical components. Wash thoroughly after handling. Avold conlac with eyee.

FAAGILE: Liquid in gless. Handlo with care.

Product should be stored in the datk and st room femperature; however, temperatures up $10120^{\mathrm{h}} \mathrm{F}$ or even below freazing will not normally aftect raagent pertormance.

\section{EXPOSURE CONTAOLSI PEASONAL PROTECTION}

OSHA \& ACGIH THAESHOLD LUMIT VALUE: $5 \mathrm{ppm}$ HydrochloHC ACId PROTECTIVE EQUIPMENT: Safoty glassos

\section{PHYSICAL AND CHEMICAL PROPERTIES}

STATE: LlquId APPEARANCE: Colortaes ODOR: Pungent

SOLUBILTY IN WATER: Misciblo pH: $<1$

BOLLING POINT: $150^{\circ} \mathrm{C}$ MELTING POINT: N/A

VAPOR FFESSURE: N/A SPECIFIC GRAVITY: 1.2

VAPOA OENSTTY: NA

\section{$X$ STABILITY AND REACTIVITY}

HAZARDOUS DECOMPOSTION PAODUCTS: Emite toxle fumes. Heating releases horeasing amounis of hydrogen chlonido.

\section{TOXJCOLOGICAL INFORMATION}

Carcinogen Statue (Hydrochlortc Acld): ACGIH: (A2) - suspected human carsinogen NTP: Suspect carcinogen

OSHA: Possible select cardnogan

IARC: Group 3 cardnogen

\section{ECOLOGICAL INFORMATION}

Environmentel Fate: Substance will neutralize soll carbonate-based components.

\section{DISPOSAL CONSIDERATIONS}

Diapose of in a manner consietent with Foderal, Stale and Local Pegulationg. Not listad as a malerial banned lrom lend disposal according to RCFA.

\section{TRANSPORT INFORMATION}

US DOT: 49 CFR, 173.4 Exceptions For Small Quantides

IATA: Dangerous Goods In Excopted Quantities

Hezard Clase' 8

UN Number: UN 1788

\section{$X V$. REGULATORY INFORMATION}

EUROPEAN INFORMATION:

Riak Phrases:

Corrostve. Causes Bumb. Irritating to Resplatory System

Saloty Phrasos:

In case of contact with ayes, inse immodiately with plenty of water and seax medical advices.

In case of accident or il you feel unwell, soek medcal advice immedately (show label where posstble).

U.S. INFOAMATION:

All chemleal componente of this product are listed on the TSCA inventory. This material contalns hydrochloric acid which is subled to the reporting requilements of Section 313 of SARA TMle 111 and 10 CFA $\$ 73$.

\section{OTHER INFORMATION}

THE ABOVE INFOAMATIOWIS BELIEVEO TO BE NCEURATE ANO REPRESENTS THE BEST NPOAMMTION CLRAENTY AVAILALL TO UE. NLL PAOOUCTS APE OFFERED IN ACCOPOANCE WTTH THE MANUFACTUAETR C CUFAENT PACOUCTION SPECIFICATIONO AND AAE INTENOEO SOLELY FOA USE IN ANAL YTICAL TESTING THE NUNUFACTUAEA SHALI IN NO EVENT BE LIABLE FOR ANY INUUAY, LOSS OR DAMASE RESULTINO FAOM THE HANOLINO, USE OR MISUSE OF THESE FAOOUCTS.

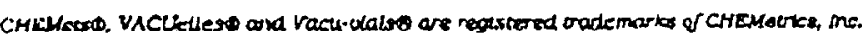


Suifuric Acid
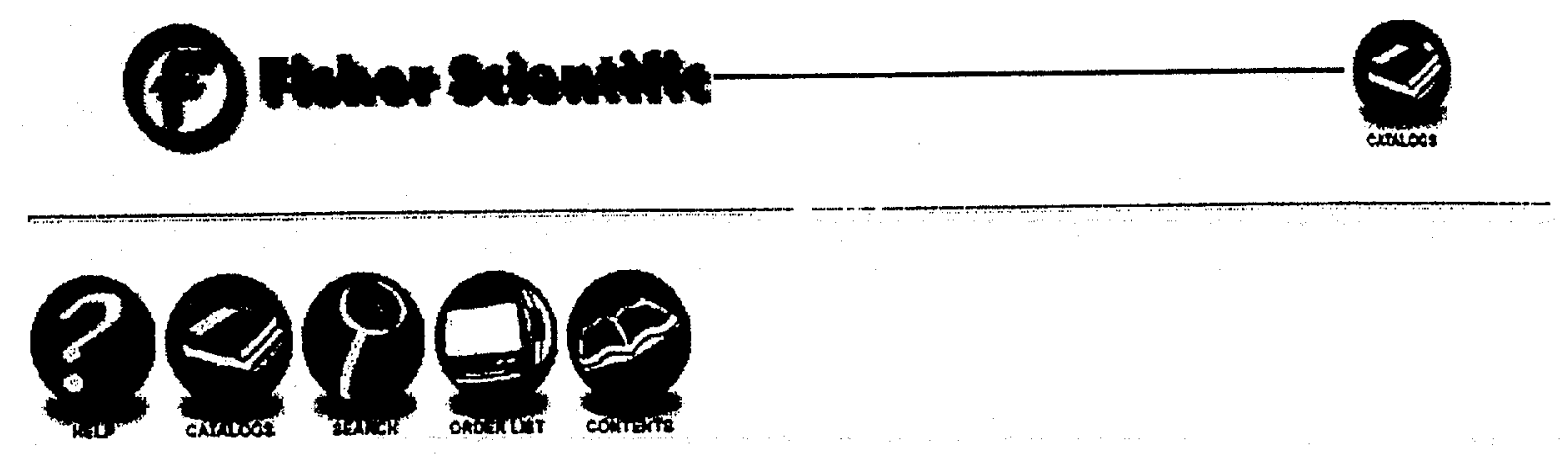

Use your web browser's "Back" key to return to previous topic.

\section{Sulfuric Acid}

$* * * *$ MATERIAL SAFETY DATA SHEET $* * * *$

\section{Sulfuric Acid}

22350

*** SECTION 1 - CHEMICAL PRODUCT :NL : OMPANY IDENTIEICATION ***

MSDS Name: Sulfuric Acid

Catalog Numbers:

S71211, S71211SC, S71826, S79200, S8Ci13, S80213-1, A298 212, A298212,

A $300212, A 300225 \mathrm{LB}, \mathrm{A} 300500, A 300$ Gil2GAL, A300 700LB, A300212, A300225LB

A300500, A300612GAL, A300700LB, A300C 2:2, A300C212, A300C212001,

A300C212002, A300C212003，A300C212004, A300C212005, A.300C212006,

$\mathrm{A} 300 \mathrm{C} 212007, \mathrm{~A} 300 \mathrm{C} 212008, \mathrm{~A} 300 \mathrm{C} 212 \mathrm{COS}, \mathrm{A} 300 \mathrm{C} 212010, \mathrm{~A} 300 \mathrm{C} 212 \mathrm{LC}, \mathrm{A} 300 \mathrm{EP}$ 500,

A.300EP500, A300J500, A300P 500, A300Fi, (1) A.300S 212, A300S 500, A300S212,

A300S212LC, A300S500, A300SI 212, A3CUSI2:2, A468-1, A468-250, A468-500,

A484 212, A484212, A510-212, A510-50C. AC3000S212002, NC9466102, NC9499595.

S71211ME, S71211MF*, S71211SCME, S71: LSCME*, S7920OME, S7920OMF* DHAZ, S7920OMF`DHAZ, S79200SCME, SA174212, 3\%1/44, SA174212, SA1744, SA1764, SA1764, SA196 500, SA196500

Synonyms:

Hydrogen Sulfate, Oil of Vitriol, Vit :i!l Brown Oil, Matzing Acid,

Battery Acid

Company Identification: Eisher Scientifi,

1 Reagent Lane

Eairlawn, NJ 07.13

For information, call: 201-796-7100

Emergency Number: 201-796-7100

For CHEMTREC assistance, cal1: 800-424-931)

For International CHEMTREC assistance, ca. : 723-527-3887

*** SECTION 2 - COMPOSITION, II FO-MATION ON INGREDEENTS ***

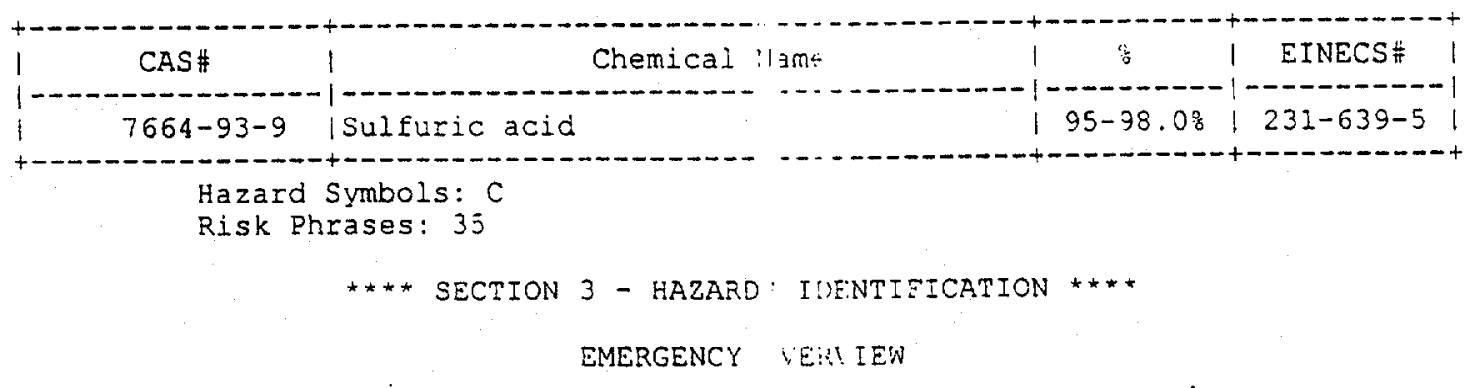


Appearance: colourless.

Danger! Strong oxidizer. Contact with other material may cause a

fire. Corrosive. Harmful if swallowed. Caus: ss eye and skin burns. May

cause severe respiratory and digestive trai": iritation with possible

burns.

Target Organs: None.

Potential Heaith Effects

Eye:

Skin:

Causes severe eye curns. May ca ise irreversible eye injury.

Ingestion:

Causes skin burns.

May cause severe and permanent dimage to the digestive tract. Causes gastrointestinal tract burns.

Inhalation:

Harmful if inhaled. Nay cause severt irritation of the respiratory tract with sore throat, coughing, shortness of breath and delayed Chronic: lung edema. Causes chemical burn $t$, the respiratory tract.

Prolonged or repeated inhalation may cause nosebleeds, nasal congestion, erosion of the teeth. perforation of the nasal septum, chest pain and bronchitis. Prolc uti or repeated eye contact may cause conjunctivitis.

*** SECTION 4 - EIRST A3) MEASURES ****

Eyes:

Flush eyes with plenty of water io: th least 15 minutes, occasionaliy lifting the upper am l wer lids. Get medical aid Skin: immediately. Do NOT allow victin $\because$ ub or keep eyes closed.

Get medical aid immediately. Fil:in skin with plenty of soap and water for at least 15 minutes wt. Le: cemoving contaminated clotining and shoes. SPEEDY ACTION IS CRII TAL

Ingestion:

Do NOT induce vomiting. If victin .s conscious and alert, give 2-4 cupfuls of milk or water. Never tre anything by mouth to an

Inhalation: unconscious person. Get medical li: immediately.

Get medical aid immediately. Ren.uvt Erom exposure to fresh ai: immediately. If not oreating, a : vt zrtificial respiration. If breathing is difficult, give ox $1 \geqslant$. DO NOT use mouth-to-mouth respiration.

Notes to Physician:

Treat symptomatically and suppo. -i: $\in$ Ly.

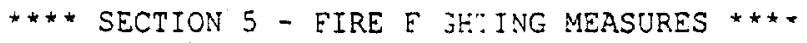

General Information:

Strong oxidizer. Contact with cumbustible materiais may cause a fire. Wear appropriate protective llothing to prevent contact with skin and eyes. Wear a self-continte breathing apparatus (SCBA) to prevent contact with thermal de omposition products. Contact with water can cause violent liberat:on ct heat and splattering of the material. Contact with metals $m i y$ rolve flammable hydrogen gas. Containers may explode when hea ec (I l E contaminatec with water. Extinguishing Media:

Use carbon dioxide or dry chemi al Do NOT get water inside containers. Cool containers wil: t coding quantities of water until well after fire is out.

Autoignition Temperature: Not availa: : $\epsilon$

Elash Point: Not available.

(estimated) Health: 3; Flammability: 0; R.ec...vicy: 2

Explosion Limits, Lower: Not availat. 6 . 


\author{
Upper: Not availabl: . \\ *** SECTION 6 - ACCIDENTA: REIEASE MEASURES ****
}

General Information: Use propex perso: 1 frotective equipment as indicated in Section 8 .

Spilis/Leaks:

Clean up spills immediately, obs".rvir.g precautions in the Protective Equipment section. Cover with sa:a, wy lime or soda ash and place in a closed container for disposal. Is: water spray to reduce vapors, do not put water directly on leak, $F i$ : area or inside container.

$$
\text { *** SECTION } 7 \text { - HANDL: } 6 \text { ir.d STORAGE } * \star \star \star
$$

Handling:

Wash thoroughly after handling. iemuve contaminated clothing and wash before reuse. Do not get in syts, on skin, or on clothing. Keep container tightly closed. Do not irigust or inhale. Do not allow contact with water. Use only in cimmical fume hood.

Storage:

Do not store near combustible ma eriuls. Keep container closed when not in use. Store in a cool, dry, we. 1-ventilated area away from incompatible substances. Do not ic... near alkaline substances.

*** SECTION 8 - EXPOSURE CONTRC:.;, 'ERSONAL PROTECTION ***

Engineering Controls;

Use process enclosure, local ext:ust ventilation, or other engineering controls to control lisoinne levels below recommended exposure limits.

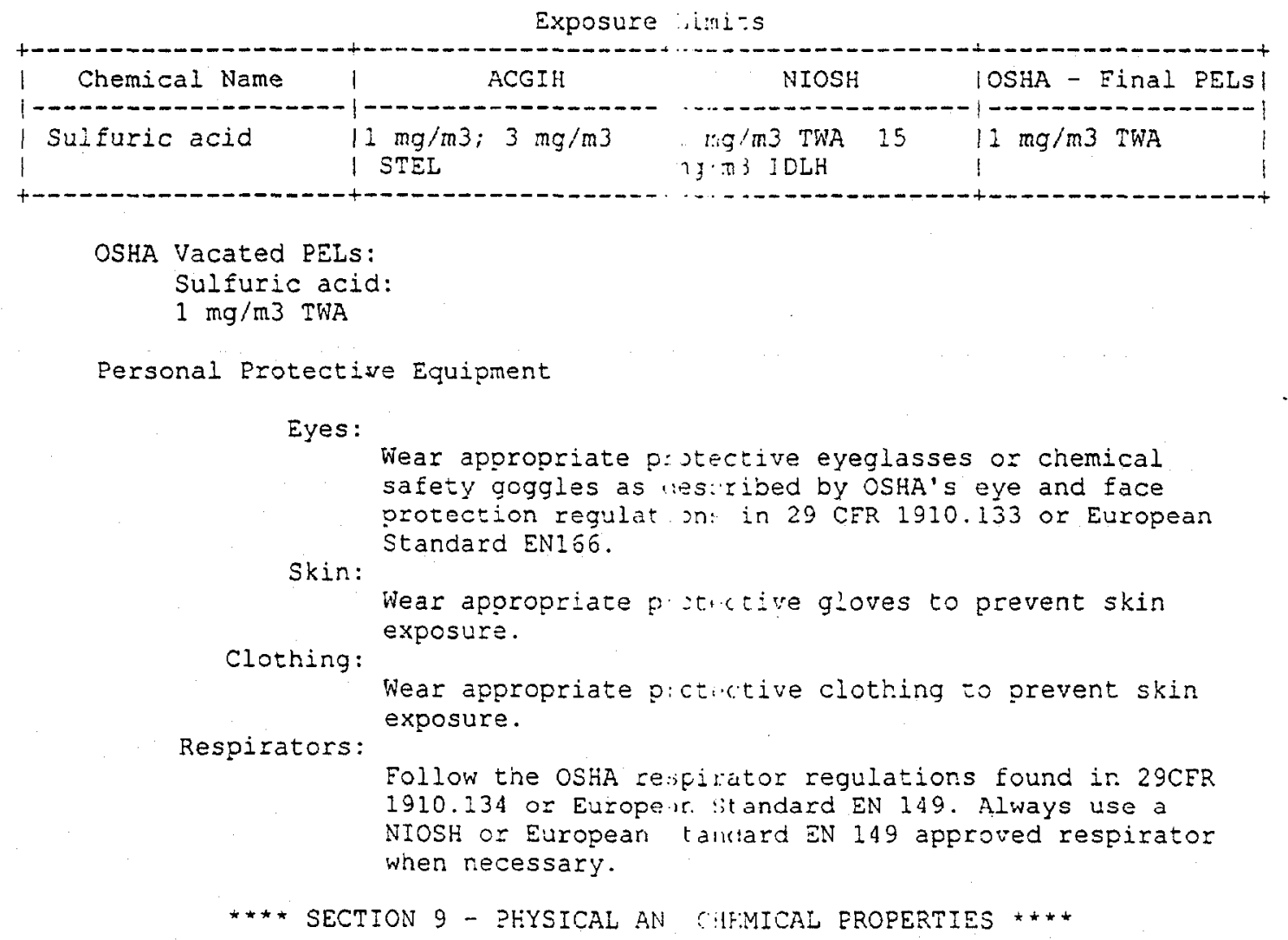

Physical State:

Liquid 


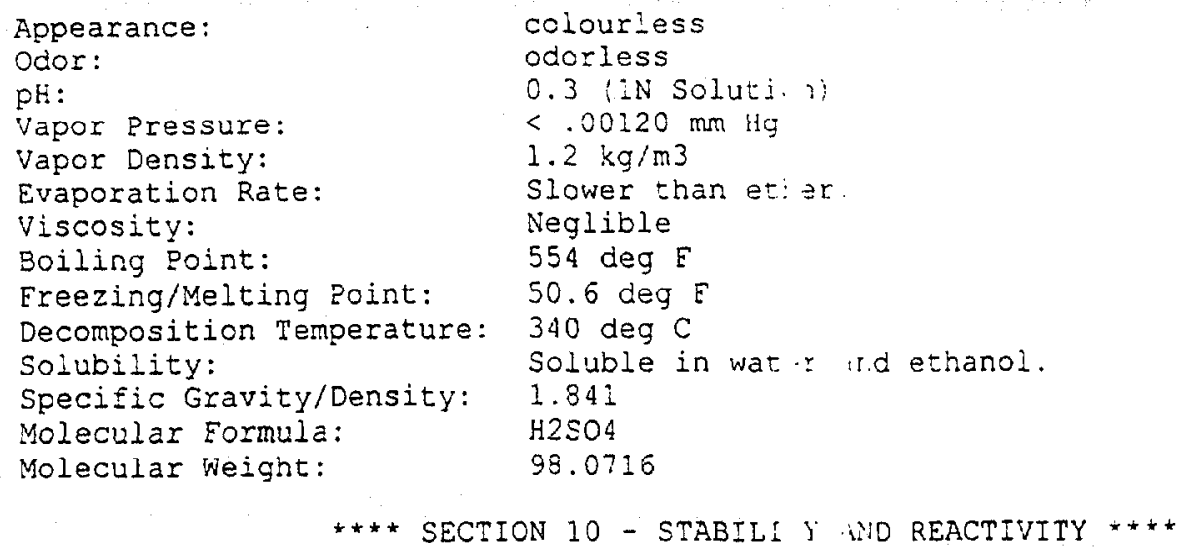

Chemical Stability: Stable under normal temperatures ar.i pressures.

Conditions to Avoid: Incompatible materiais, contact ittn water, metals, excess heat, combustible materials, organic mtes.aıs, oxidizers, amines, bases. Incompatibilities with other Material: Acetic Anhydride, Acetone Cyanhytria. Acetone + Nitric Acid, Acetone + Potassium Dichromate, Acetonitı 1 e + sulfur trioxide, Acrolein, Acrylonitrile, Alcohols + Hydrog:n Euroxide, Allyl Alcohol, Allyl Chloride, 2-Aminoethanol, Ammoni.un fydroxide Ammonium iron( III) sulfate dodecahydrate, Ammonium risurchrcmate, Aniline + Glycerol + Nitrobenzene, Benzyl Alcohol, B: Micis + Metals, tert-Butyl-m-xylene + Nitric Acid, 1-Chloro-2,3-epoypusuane, Bromine Pentafluoride, n-Butyraldehyde, Carbices, Cesili:! datylene Carbide, Chlorates, 4-Chloronitrobenzene + sulfur ty -uxile, Copper, Dichloromethane + Ethanol + Nitrate or Nitrite, 2-6yan-4-nitrobenzenediazonium hydrogen sulfate, 2-cyano-2-propanul. Chlorine Trifluoride, Chlorosulfonic Acid, Cycolpentacliere, Cyclopentanone oxime, 1.3-Diazidobenzene, Diethylamins. Uurous Nitride, Diisobutylene, Epichlorohydrin, Ethylene Cyancildin, Ethylene Diamine, Ethylene Glycol, Dimethoxydinitroanthraqt. .a. n:, 4-Dimethylaminobenzaldehyde, 2,5-Dinitro-3-Methylbenzoic acic: + Ssdium Azide, 1,5-Diritronaphthalene + Sulfur, E-noxylated nonylphenol, Hexalithium disilicide, Ethylenimine, Eulmil. tus. Other Acids, Iodine Heptafluoride, Metals, Isoprene, $\vec{i}$ crofluoric acid, Eydrogen peroxide, Metal acerylides or ciscocos, Metal Chiorates, Metal Perchlorates, 4-Mechylpyridine, Ji: amide, Nitric Acid + Organic Materials, Nitric Acid + Toluen, izroaryl bases and derivatives, Nitrobenzene, 3-Nitrobenzenesul: zn:c Acid, Nitromethane, N-Nitromethylamine, 4-Nitrotolue re, Permanganates, Phosphorus, Phosphorus (III) Oxide, Polyisi $i$-re), Mercuric Nitride, Mesityl Oxide, P-Nitrotoluene, Pentasil or Irihydroxydiaminophosphate, Perchlorates, Permanganates + $B_{1} \cdot 7 z$ re, Phosphorus Isocyanate, Picrates, Potassium t-Butoxide, Fo assiun, 3-Propynol, Potassium Chlorate, Permanganates, beta-p sp.clactone, Eropylene Oxide, Pyridine, Rubidium Acetylene Ca oi it and Sodium, Silver Rermanganates, Silver Peroxochr ma: $\epsilon$, Sodium, Sodium Carbonate, Sodium Tetrahydroborate, Sodium Th cyanate, Sucrose, Tetramethylbenzenes, 1,2,4,5-ie i a : ne, Thallium (I) azidodithiocarbonate, 1, 3,5-T.: :" sohexahydro-1,3,5-triazine, Water, and 2 inc Iodide.

Hazardous Decompcsicion Products: Oxides of sulfur.

Hazardous Polymerization: Has not be. $r$ rported.

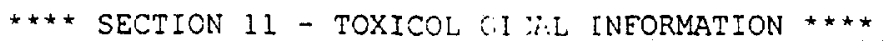

RTECS\# : 


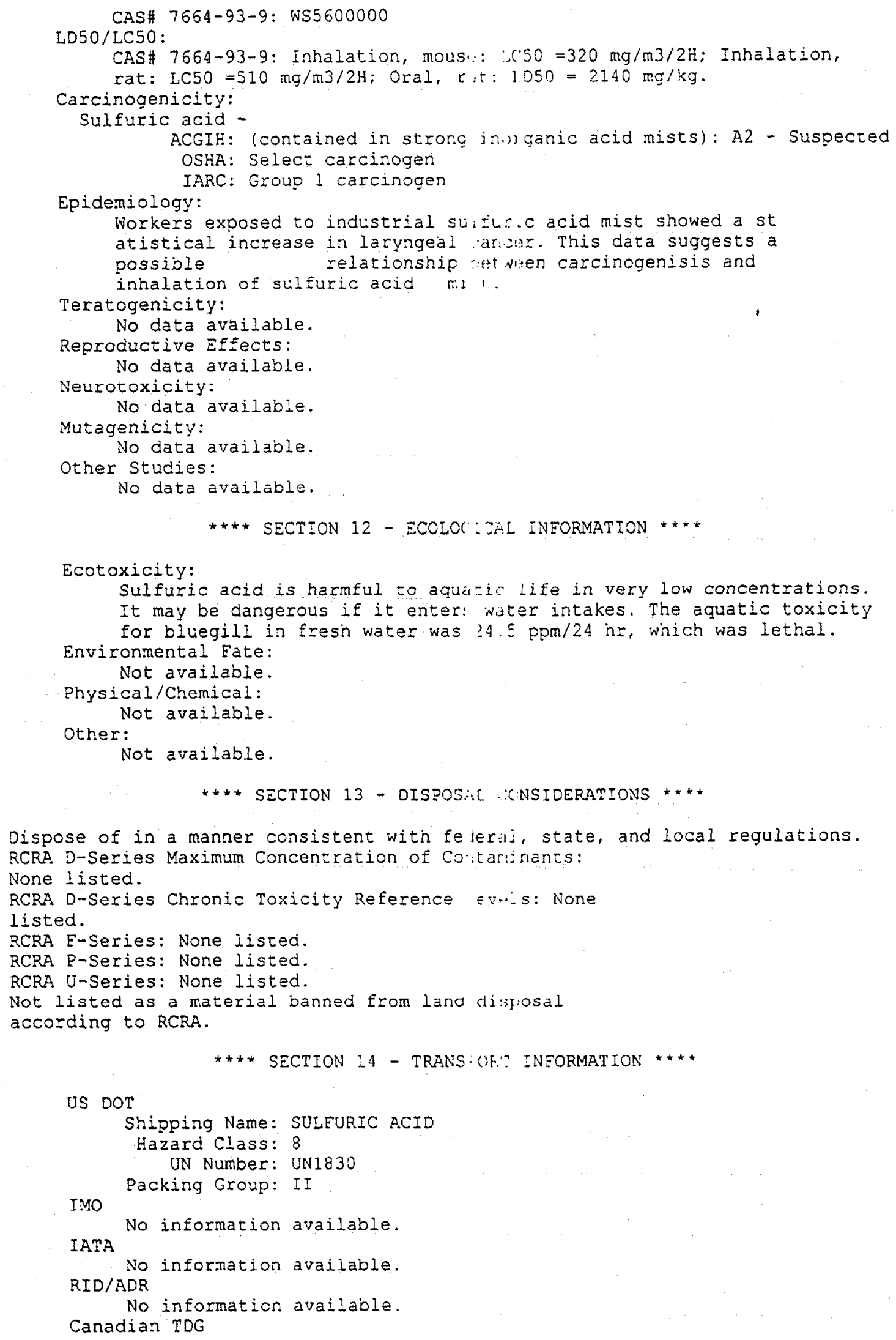


Shipping Name: SULEURIC ACID

Hazard Class: $8(9,2)$

UN Number: UN1830

*** SECTION 15 - REGULA:CR $\because$ INEORMATION ***

US TSCA

CAS\# 7664-93-9 is listed on the ISCA inventory.

Health \& Safety Reporting List None of the chemicals are on the Her.th \& Safety Reporting List.

Chemical Test Rules None of the chemicals in this pr du: are under a Chemical Test Rule. Section $12 b$ None of the chemicals are listec 11 ind TSCA Section $12 \mathrm{~b}$.

TSCA Significant New Use Rule SARA None of the chemicats in this ma' in: have a SNUR under TSCA.

Section 302 (RQ)

CAS\# 7664-93-9: final $R Q=1000$ (nunis $(454 \mathrm{~kg})$

Section 302 (TPQ)

CAS\# 7664-93-9: TPQ $=1000$ pouns:3: R2 $=1000$ pounds

SARA Codes

CAS \# 7664-93-9: acute, chronic, raazive.

Section 313

This material contains Sulfuric 10id (CAS\# 7664-93-9, 9598 0\%), which is subject to the reporting regt. rements of section 313 of SARA Title III and 40 CER Part 373.

Clean Air Act:

This material does not contain iny razardous air pollutants.

This material does not contain : :y class 1 Ozone depletors.

This material does not contain ... iy Class 2 Ozone depletors.

Clean Water Act:

CAS\# 7664-93-9 is listed as a Hkza:cous Substance under the CWA. None of the chemicais in this $p$ od..ct are listed as Priority Pollutants under the CWA. None of the chemicals in this $p$ xi: ct are listed as Toxic Pollutants under the CWA.

OSHA:

None of the chemicals in this $p$ sdict are considered highly hazardous by OSHA.

STATE

Sulfuric acid can be found on the fo lointing state right to know

lists: California, New Jersey, Flor: : . Fernsylvania, Minnesota, Massachusetts.

California No Significant Risk Level

None of the chemicals in this produc a:"t Iisced.

European/International Reguiations

European Labeling in Accordance with EC lirectives

Hazard Symbols: C

Risk Phrases:

Safety Phrases:

R 35 Causes sever. tins.

S 26 In case of c or aut with eyes, rinse immediately

with plenty of wat:r and seek medical acivice.

530 Never add wá: ex: O this product.

545 In case of $a$ a 1 . nt of if you feel unwell, seek medical advice imn ' $1: 1$ ely (show the label where possiblel.

WGK (Water Danger/Protection)

CAS\# 7664-93-9:2

Canada

CAS\# 7664-93-9 is listed on Car.uda'; DSL/NDSI List.

This product has a WHMIS classilicarion of $C, D I A, E$.

CAS\# 7664-93-9 is not listed or Cunda's Ingredient Disclosure List. 
Exposure Limits

CAS\# 7664-93-9:. OEL-ARAB Repub. ic cf Egypt:TWA $1 \mathrm{mg} / \mathrm{m} 3$. OEL-AUSTRALI A. TWA $1 \mathrm{mg} / \mathrm{m3}$. OEL-BELGIUM:TWA $1 \mathrm{ng} / \mathrm{m} 3 ;$ STEL $3 \mathrm{mg} / \mathrm{m} 3$. OEL-CZECHOSLOVAKI A:TWA $1 \mathrm{mg} / \mathrm{m} 3$;STEL $2 \mathrm{mg} / \mathrm{m3}$. OEL-VENMARK:TWA $1 \mathrm{mg} / \mathrm{m} 3$. OEL-FINLAND:TWA I $\mathrm{ng} / \mathrm{m} 3$; STEL $3 \mathrm{mg} / \mathrm{m} 3$;SKIn. OEL-ERAVICE:TNA $1 \mathrm{mg} / \mathrm{m} 3 ; \mathrm{STEL} 3 \mathrm{mg} / \mathrm{m} 3$. OEL-GER MANY: TWA $1 \mathrm{mg} / \mathrm{m} 3$, OEL-HUNGARY:S?:: $: \mathrm{mg} / \mathrm{m} 3$. OEL-JAPAN: TWA $1 \mathrm{mg} / \mathrm{m} 3$

-THE NETHERLANDS: TWA $1 \mathrm{mg} / \mathrm{m} 3$. OE:.-THE. PHILIEPINES:TWA $1 \mathrm{mg} / \mathrm{m} 3$. OEL-EOL AND:TWA $1 \mathrm{mg} / \mathrm{m} 3$. OEL-RUSSIA:STEL 1 :G/m3:Skin. OEL-SWEDEN:TWA $1 \mathrm{mg} / \mathrm{m} 3$; STEL $3 \mathrm{mg} / \mathrm{m} 3$. OEL-SWITZERLAND:TWi: i $\mathrm{mg} / \mathrm{m} 3 ;$ STEL $2 \mathrm{mg} / \mathrm{m} 3$. OEL-THAILAND:T WA $1 \mathrm{mg} / \mathrm{m} 3$. OEL-IURKEY:TWA I $\mathrm{mg} / . \vdots$. (ES-UNITED KINGDOM:TWA $1 \mathrm{mg} / \mathrm{m} 3$ L IN BULGARIA, COLOMBIA, JORDAN, GCRHA nECK ACGIH TLV. OEL IN NEN ZEA LAND, SINGAPORE, VIETNAM check i :i! '"LV

**** SECTION 16 - ADDITI NA. INEORMATION ****

MSOS Creation Date: 12/28/1994 Revis, or. "23 Date: 12/12/1997

The information above is believed to : weurate and represents the best information currently available to us H jwever, we make no warranty of merchantability or any other warranty, expzess or implied, with respect to such information, and we assume no i.bility resulting from its use. Users should make their own investigations, derermine the suitabilicy of the information for their particular purp :ins. In no way shall Fisher be liable for any claims, losses, or damages 0 : 1ny third party or for lost profits or any speciai, indirect, incidentai, :niequential or exemplary damages, howsoever arising, even it i s: e- has been advised of the possibility of such damages. 
MATERIAL SAFETADDATA SHEET (MSDS) MATERIAL SAFETY DATA SHEET

EM SCIENCE

(124) 20899

\section{CHEMICAL PRODUCT AND COMPANY IDENTIFICATION}

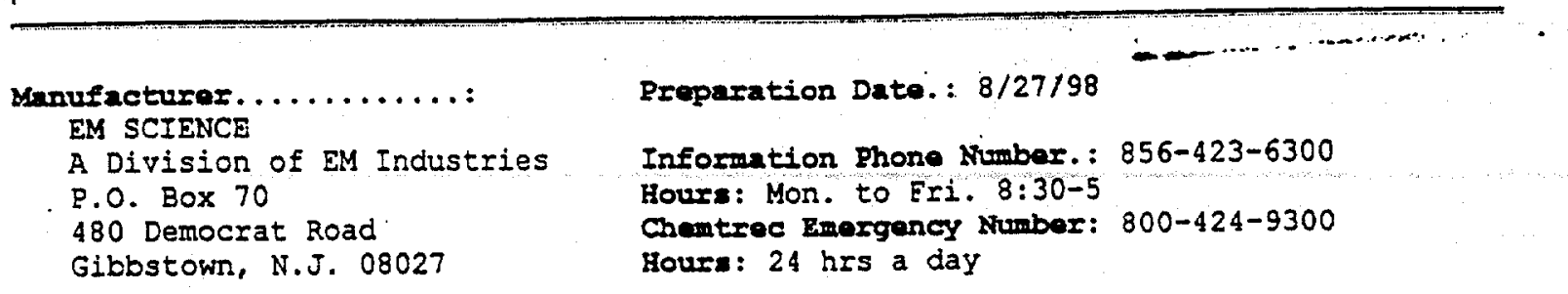

Catalog Number(s):

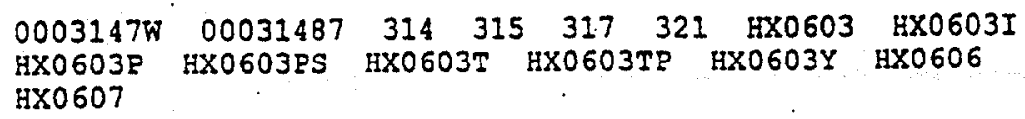

Product Name:

Hydrochloric Acid

Synonyms:

Muriatic Acid, 23 deg. Be

Chemical Family:

Inorganic Acid

Formula:

$\mathrm{HCl}$

Molecular Weight.:

36.46

\section{COMPOSITION / INFORMATION ON INGREDIENTS}

Component
Hydrochloric Acid
CAS \# $7647-01-0 \quad 1008$
Approximate per cent indicates that this product is a
concentrated acid. Hydrochloric acid is approximately 378.
3. HAZARDS IDENTIFICATION

EMERGENCY OVERVIEW

CAUSES SEVERE BURNS.

MAY BE FATAL IF INHALED OR SWALLOWED.

VAPOR EXTREMELY IRRITATING.

May Cause Damage To Respiratory Passages and Lungs.

Appearance:

Clear, colorless liquid; acrid odor 


\section{POTENTLAL HEALTH EFFECTS (ACUTE AND CHRONIC)}

\section{Symptoms of Exposure:}

Eye contact causes severe burns and permanent eye damage. Skin contact causes severe burns, possible deep ulceration. Inhalation causes damage to nasal and respiratory passages, pulmonary edema. Ingestion causes burns of mouth, throat and gastrointestinal tract.

\section{Medical Cond. Aggravated by Exposure:}

Respiratory conditions

\section{Routes of Entry:}

Inhalation, ingestion or skin contact.

\section{Carcinogenicity:}

The material is not listed (IARC, NTP, OSHA) as cancer causing agent.

\section{FIRST AID MEASURES}

\section{Emergency First Aid:}

GET MEDICAL ASSISTANCE FOR ALI CASES OF OVEREXPOSURE.

Skin: Immediately flush thoroughly with large amounts of water.

Eyes: Immediately flush thoroughly with water for at least 15 minutes.

Inhalation: Remove to fresh air; give artificial respiration if

breathing has stopped.

Ingestion: do not induce vomiting; give water or milk if conscious;

get medical attention.

\section{FIRE FIGHTING MEASURES}

Flash Point (F): Noncombustible

Flammable Limits LEL (\%): N/A

Flammable Limits UEL (\%): N/A

Extinguishing Media:

Water spray

Fire Fighting Procedures:

Wear self-contained breathing apparatus and protective clothing.

Fire \& Explosion Hazards:

Contact with metals produces hydrogen which may form explosive mixtures with air.

\section{ACCIDENTAL RELEASE MEASURES}

\section{Spill Response:}

Evacuate the area of all unnecessary personnel. Wear suitable protective equipment listed under Exposure / Personal Protection. Eliminate any ignition sources until the area is determined to be free from explosion or fire hazards. Contain the release and eliminate its source, if this can be done without risk. Take up and containerize for proper disposal as described under Disposal. Comply with Federal, State, and local regulations on reporting releases. Refer to Regulatory Information for reportable quantity and other regulatory data. EM SCIENCE recommends SPILL-X neutralizers and absorbent agents for various types of spills. 
Additional information on the SPILL-X products can be provided through the EM SCIENCE Technical Service Department (609) 423-6300. The following EM SCIENCE SPILL-X neutralizer and absorbent is recommended for this product:

Sx0861 Spill-X-A Acid Spill Treatment Kit

\section{HANDLING AND STORAGE}

Handling \& Storage:

Keep container tightly closed. Store in a cool, well-ventilated area separated from incompatible materials. Do not breathe vapor. Do not get in eyes, on skin, or on clothing. Handle empty containers with caution. Add acid cautiously to water when diluting - never add water to acid.

\section{EXPOSURE CONTROLS / PERSONAL PROTECTION}

\section{ENGINEERING CONTROLS AND PERSONAL PROTECTIVE EQUIPMENT:}

Ventilation, Respiratory Protection, Protective Clothing, Eye Protection:

Respiratory Protection: If workplace exposure limit(s) of product or any component is exceeded (see T/V/PEL), a NIOSH/MSHA approved air supplied respirator is advised in absence of proper -nvironmental control. OSHA regulations also permit other NIOSH/MSHA respirators (negative pressure type) under specified conditions (see your safety equipment supplier). Engineering and/or administrative controis should be implemented to reduce exposure. Material must be handled or transferred in an approved fume hood or with equivalent ventilation. Protective gloves (Neoprene, Nitrile or equivalent) must be worn to prevent skin contact. Safety glasses with side shieids must be worn at all times.

\section{Work/Hygenic Practices:}

Wash thoroughly after handling. Do not take internally. Eye wash and safety equipment should be readily available.

\section{EXPOSURE GUIDELINES}

OSHA - PEL:

Component PRM ${ }_{\text {MG/M3 PPM }}^{\text {STEL }}$ MG/M3 PPM CL MG/M3 Skin

Hydrochloric Acid

$5 \quad 7$

\begin{tabular}{|c|c|c|c|c|c|c|c|}
\hline \multirow[b]{2}{*}{ Component } & \multicolumn{2}{|c|}{ TWA } & \multicolumn{2}{|c|}{ STEL } & \multicolumn{2}{|c|}{ CL } & \\
\hline & PPM & MG/M3 & PPM & MG/M3 & PPM & MG/M3 & Skin \\
\hline Hydrochlo & & & 5 & 7.5 & 5 & 7.5 & \\
\hline
\end{tabular}

If there are no exposure limit numbers listed in the Exposure Guidelines chart, this indicates that no OSHA or ACGIH exposure limts have been established.

\section{PHYSICAL AND CHEMCIAL PROPERTIES}


soiling Point (C $760 \mathrm{mmHg}$ : $110 \mathrm{C} 20.24 \%$

Melting Point (C) : -74C

Specific Gravity $\left(\mathrm{H}_{2} \mathrm{O}=1\right): 1.1885$

Vapor Pressure (mm Hg) : $16020 \mathrm{C}$

Percent Volatile by vol (\%): $99.9+\%$

Vapor Density $(\mathrm{Air}=1): 1.25$

Evaporation Rate (BuAc = 1): 2.0

Solubility in Water (\%): Miscible

Appearance :

Clear, colorless liquid; acrid odor

\section{STABILITY AND REACTIVITY}

Stability: Yes

Hazardous Polymerization:

Does not occur

Hazardous Decomposition:

Toxic hydrogen chloride gas

Conditions to Avoid:

Heat; contact with metals.

\section{Materials To Avoid:}

( ) Water

() Acids

(X) Bases

() Corrosives

() Oxidizers

(x) Other: Alkalies and amines; contact with metals

produces hydrogen

\section{TOXICOLOGICAL INFORMATION}

\section{Toxicity Data}

IhI-hms LDLO: $1300 \mathrm{ppm} / 30 \mathrm{M}$

orl-rbt LD50: $900 \mathrm{mg} / \mathrm{kg}$

\section{Toxicological Findings:}

Test on laboratory animals indicate material may produce adverse mutagenic and reproductive effects.

Cited in Registry of Toxic Effects of Chemical Substances (RTECS)

\section{DISPOSAL CONSIDERATIONS}

EPA Taste Numbers: D002 
reatment:

Specified Technology - Neutralize to $\mathrm{pH}$ 6-9. Contact your local permitted waste disposal site (TSD) for permissible treatment sites.

ALWAYS CONTACT A PERMITTED WASTE DISPOSER (TSD) TO ASSURE COMPLIANCE

WTTH ALL CURRENT LOCAL, STATE AND FEDERAL REGULATIONS.

\section{TRANSPORT INFORMATION}

DOT Proper Shipping Name:

Hydrochloric Acid

DOT ID Number :

UN1789

\section{REGULATORY INFORMATION}

\section{TSCA Statement:}

This product is a "Mixture". The CAS numbers of all components are listed on the TSCA Inventory.

\begin{tabular}{lccc} 
Component & $\begin{array}{c}\text { SARA } \\
\text { EHS } \\
(302)\end{array}$ & $\begin{array}{c}\text { SARA } \\
\text { EES TPQ } \\
\text { (Ibs) }\end{array}$ & $\begin{array}{c}\text { CERCLA } \\
\text { RQ } \\
(1 b s)\end{array}$ \\
\hline Hydrochloric Acid & $Y$ & 500 & 5000 \\
Component & Eloor List & $\begin{array}{c}\text { SARA } \\
313\end{array}$ & $\begin{array}{c}\text { DeMinimis } \\
\text { for SARA 313 } \\
(8)\end{array}$ \\
\hline Eydrochioric Acid & $Y$ & $Y$ & 1.0
\end{tabular}

If there is no information listed on the regulatory information chart, this indicates that the chemical is not covered by the specific regulation listed.

\section{OTHER INFORMATION}

\section{Comments:}

None

NFPA Hazard Ratings:

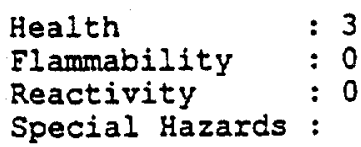


1 - Revised Section

N/A = Not Available

N/E - None Establised

The statements contained herein are offered for informational purposes only and are based upon technical data that EM Science believes to be accurate. It is intended for use only by persons having the necessary technical skill and at their own discretion and risk. Since conditions and manner of use are outside our control, we make NO WARRANTY, EXPRESS OR IMPLIED, OR

MERCHANTABILITY, FITNESS OR OTHERWISE. 


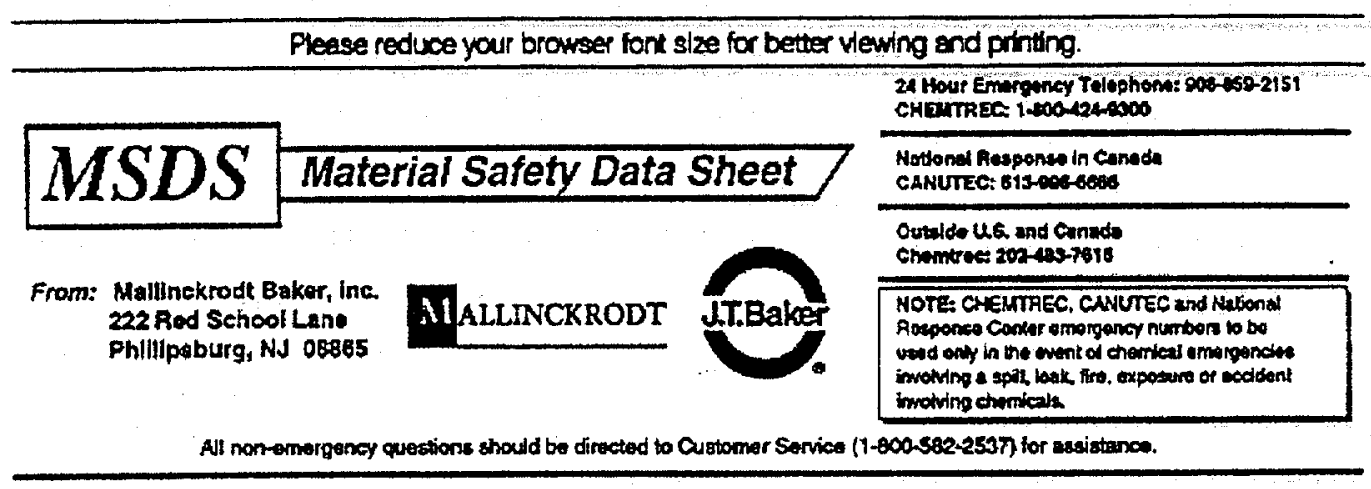

\section{FORMALDEHYDE}

MSDS Number: F5522 --- Effective Date: 03/23/98

\section{Product Identification}

Synonyms: Formaldehyde 37\%; Formalin; Morbicid Acid; Methylene Oxide; Methyl aldehyde

CAS No.: 50-00-0

Molecular Weight: 30.03

Chemical Formula: $\mathrm{HCHO}$ and $\mathrm{CH} 3 \mathrm{OH}$ in water

Product Codes:

J.T. Baker: 2105, 2106, 2107

Mallinckrodt: 5014,5016

\section{Composition/Information on Ingredients}

\begin{tabular}{|c|c|c|c|}
\hline Ingredient & CAS No & Percent & Hazardous \\
\hline 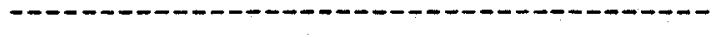 & $-------m--$ & $-\infty-\infty-\infty$ & $-----\infty-n$ \\
\hline $\begin{array}{l}\text { Eormaldehyde } \\
\text { Methyl Alcohol } \\
\text { Water }\end{array}$ & $\begin{array}{l}50-00-0 \\
67-56-1 \\
7732-18-5\end{array}$ & $\begin{array}{c}37 \% \\
10-15 \% \\
48-53 \%\end{array}$ & $\begin{array}{l}\text { Yes } \\
\text { Yes } \\
\text { No }\end{array}$ \\
\hline
\end{tabular}

\section{Hazards Identification}

Emergency Overview

POISON! DANGER! SUSPECT CANCER HAZARD. MAY CAUSE CANCER. Risk of cancer depends on level and duration of exposure. VAPOR HARMFUL. HARMFUL IF INHALED OR ABSORBED THROUGH SKIN. CAUSES IRRITATION TO SKIN, EYES AND RESPIRATORY TRACT. STRONG SENSITIZER. MAY BE FATAL OR CAUSE BLINDNESS IF SWALLOWED. 


\section{CANNOT BE MADE NONPOISONOUS. FLAMMABLE LIQUID AND VAPOR.}

J.T. Baker SAF-T-DATA ${ }^{(\mathrm{tm})}$ Ratings (Provided here for your convenience)

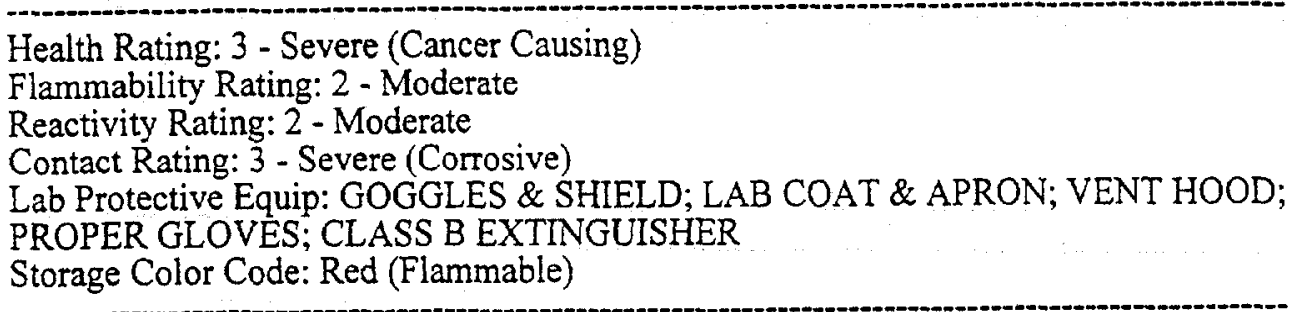

\section{Potential Health Effects}

The perception of formaldehyde by odor and eye irritation becomes less sensitive with time as one adapts to formaldehyde. This can lead to overexposure if a worker is relying on formaldehyde's warning properties to alert him or her to the potential for exposure.

\section{Inhalation:}

May cause sore throat, coughing, and shortness of breath. Causes irritation and sensitization of the respiratory tract. Concentrations of 25 to $30 \mathrm{ppm}$ cause severe respiratory tract injury leading to pulmonary edema and pneumonitis. May be fatal in high concentrations.

\section{Ingestion:}

Can cause severe abdominal pain, violent vomiting, headache, and diarrhea. Larger doses may produce decreased body temperature, pain in the digestive tract, shallow respiration, weak irregular pulse, unconsciousness and death. Methanol component affects the optic nerve and may cause blindness.

\section{Skin Contact:}

Toxic. May cause irritation to skin with redness, pain, and possibly burns. Skin absorption may occur with symptoms paralleling those from ingestion. Formaldehyde is a severe skin irritant and sensitizer. Contact causes white discoloration, smarting, cracking and scaling. Eye Contact:

Vapors cause irritation to the eyes with redness, pain, and blurred vision. Higher concentrations or splashes may cause irreversible eye damage.

Chronic Exposure:

Frequent or prolonged exposure to formaldehyde may cause hypersensitivity leading to contact dermatitis. Repeated or prolonged skin contact with formaldehyde may cause an allergic reaction in some people. Vision impairment and enlargement of liver may occur from methanol component. Formaldehyde is a suspected carcinogen (positive animal inhalation studies). Aggravation of Pre-existing Conditions:

Persons with pre-existing skin disorders or eye problems, or impaired liver, kidney or respiratory function may be more susceptible to the effects of the substance. Previously exposed persons may have an allergic reaction to future exposures.

\section{First Aid Measures}

\section{Inhalation:}

Remove to fresh air. If not breathing, give artificial respiration. If breathing is difficult, give oxygen. Call a physician.

Ingestion:

If swallowed and the victim is conscious, dilute, inactivate, or absorb the ingested 
formaldehyde by giving milk, activated charcoal, or water. Any organic material will inactivate formaldehyde. Keep affected person warm and at rest. Get medical attention immediately. If vomiting occurs, keep head lower than hips.

Skin Contact:

In case of contact, immediately flush skin with plenty of water for at least 15 minutes while removing contaminated clothing and shoes. Wash clothing before reuse. Thoroughly clean shoes before reuse. Get medical attention immediately.

Eye Contact:

Immediately flush eyes with plenty of water for at least 15 minutes, lifting lower and upper eyelids occasionally. Get medical attention immediately.

\section{Note to Physician:}

Monitor arterial blood gases and methanol levels after significant ingestion. Hemodyalysis may be effective in formaldehyde removal. Use formic acid in urine and formaldehyde in blood or expired air as diagnostic tests.

\section{Fire Fighting Measures}

Fire:

Flash point: $60 \mathrm{C}$ (140F) CC

Autoignition temperature: $300 \mathrm{C}(572 \mathrm{~F})$

Flammable limits in air \% by volume:

lel: 7.0 ; uel: 73

Flammable liquid and vapor! Gas vaporizes readily from solution and is flammable in air. Explosion:

Above flash point, vapor-air mixtures are explosive within flammable limits noted above. Containers may explode when involved in a fire.

Fire Extinguishing Media:

Water spray, dry chemical, alcohol foam, or carbon dioxide.

\section{Special Information:}

In the event of a fire, wear full protective clothing and NIOSH-approved self-contained breathing apparatus with full facepiece operated in the pressure demand or other positive pressure mode. Water may be used to flush spills away from exposures and to dilute spills to non-flammable mixtures.

\section{Accidental Release Measures}

Ventilate area of leak or spill. Remove all sources of ignition. Wear appropriate personal protective equipment as specified in Section 8. Isolate hazard area. Keep unnecessary and unprotected personnel from entering. Contain and recover liquid when possible. Use non-sparking tools and equipment. Collect liquid in an appropriate container or absorb with an inert material (e. g., vermiculite, dry sand, earth), and place in a chemical waste container. Do not use combustible materials, such as saw dust. Do not flush to sewer! US Regulations (CERCLA) require reporting spills and releases to soil, water and air in excess of reportable quantities. The toll free number for the US Coast Guard National Response Center is (800) 424-8802. If a leak or spill has not ignited, use water spray to disperse the vapors, to protect personnel attempting to stop leak, and to flush spills away from exposures. 


\title{
7. Handling and Storage
}

Store in a tightly closed container. Protect against physical damage. Store in a cool, dry weil-ventilated location, away from any area where the fire hazard may be acute. Outside or detached storage is preferred. Separate from incompatibles. Containers should be bonded and grounded for transfers to avoid static sparks. Storage and use areas should be No Smoking areas. Use non-sparking type tools and equipment, including explosion proof ventilation. Wear special protective equipment (Sec. 8) for maintenance break-in or where exposures may exceed established exposure levels. Wash hands, face, forearms and neck when exiting restricted areas. Shower, dispose of outer clothing, change to clean garments at the end of the day. Avoid cross-contamination of street clothes. Wash hands before eating and do not eat, drink, or smoke in workplace. Protect from freezing. Containers of this material may be hazardous when empty since they retain product residues (vapors, liquid); observe all warnings and precautions listed for the product.

\section{Exposure Controls/Personal Protection}

\author{
Airborne Exposure Limits: \\ -OSHA Permissible Exposure Limit (PEL): \\ $0.75 \mathrm{ppm}$ (TWA), $2 \mathrm{ppm}$ (STEL), $0.5 \mathrm{ppm}$ (TWA) action level for formaldehyde \\ $200 \mathrm{ppm}$ (TWA) for methanol \\ -ACGIH Threshold Limit Value (TLV): \\ 0.3 ppm Ceiling formaldehyde, A2 Suspected Human Carcinogen \\ $200 \mathrm{ppm}$ (TWA) $250 \mathrm{ppm}$ (STEL) skin for methanol \\ Ventilation System:
}

A system of local and/or general exhaust is recommended to keep employee exposures below the Airborne Exposure Limits. Local exhaust ventilation is generally preferred because it can control the emissions of the contaminant at its source, preventing dispersion of it into the general work area. Please refer to the ACGIH document, Industrial Ventilation, A Manual of Recommended Practices, most recent edition, for details.

Personal Respirators (NIOSH Approved):

If the exposure limit is exceeded, a full facepiece respirator with a formaldehyde cartridge may be worn up to 50 times the exposure limit or the maximum use concentration specified by the appropriate regulatory agency or respirator supplier, whichever is lowest. For emergencies or instances where the exposure levels are not known, use a full-facepiece positive-pressure, air-supplied respirator. WARNING: Air purifying respirators do not protect workers in oxygen-deficient atmospheres. Irritation also provides warning. For Methanol: If the exposure limit is exceeded, wear a supplied air, full-facepiece respirator, airlined hood, or full-facepiece self-contained breathing apparatus.

\section{Skin Protection:}

Wear impervious protective clothing, including boots, gloves, lab coat, apron or coveralls, as appropriate, to prevent skin contact.

Eye Protection:

Use chemical safety goggles and/or a full face shield where splashing is possible. Maintain eye wash fountain and quick-drench facilities in work area.

Other Control Measures:

See OSHA Standard for more information on personal protective equipment, engineering and work practice controls, medical surveillance, record keeping, and reporting requirements. (29 CFR 1910.1048)

\section{Physical and Chemical Properties}




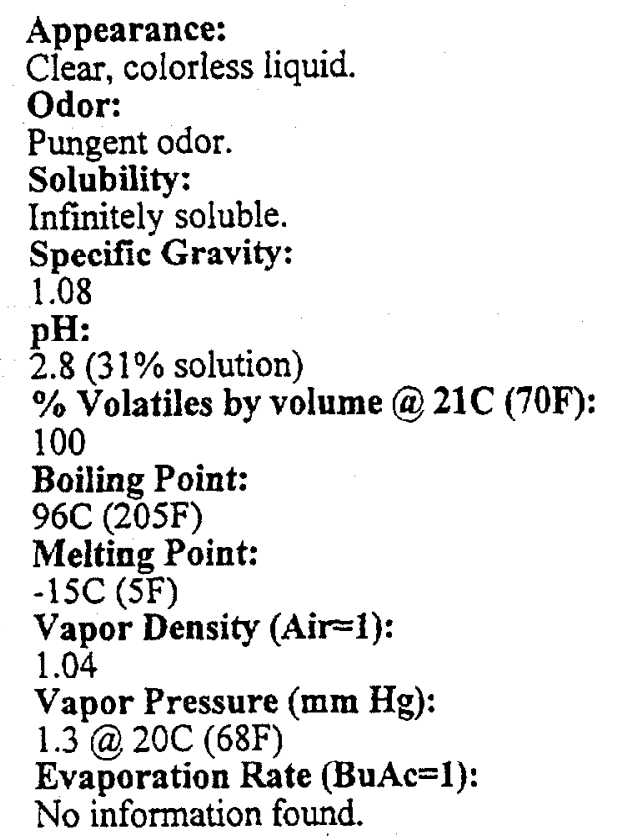

\section{Stability and Reactivity}

Stability:

Stable under ordinary conditions of use and storage.

Hazardous Decomposition Products:

May form carbon dioxide, carbon monoxide, and formaldehyde when heated to decomposition. Hazardous Polymerization:

Trioxymethylene precipitate can be formed on long standing at very low temperatures.

Nonhazardous polymerization may occur at low temperatures, forming paraformaldehyde, a white solid.

Incompatibilities:

Incompatible with oxidizing agents and alkalis. Reacts explosively with nitrogen dioxide at ca. 180C (356F). Reacts violently with perchloric acid, perchloric acid-aniline mixtures, and nitromethane. Reaction with hydrochloric acid may form bis-chloromethyl ether, an OSHA regulated carcinogen.

Conditions to Avoid:

Heat, flames, ignition sources and incompatibles.

\section{Toxicological Information}

Formaldehyde: Oral rat LD50: $100 \mathrm{mg} / \mathrm{kg}$; skin rabbit LD50: $270 \mathrm{uL} / \mathrm{kg}$, Irritation data: eye, rabbit, 750ug Severe; inhalation rat LC $50: 203 \mathrm{mg} / \mathrm{m} 3$; investigated as a tumorigen, mutagen, reproductive effector; Cancer Status: an OSHA regulated carcinogen. Methanol: oral rat LD50: $5628 \mathrm{mg} / \mathrm{kg}$; inhalation rat LC50: $64000 \mathrm{ppm} / 4 \mathrm{H}$; skin rabbit LD50: 15800 $\mathrm{mg} / \mathrm{kg}$; investigated as a tumorigen, mutagen, reproductive effector. 


$\begin{array}{llcc} & & \\ \text { Ingredient } & \text { Known } & \text { Anticipated } & \text { IARC Category } \\ \text { Eormaldehyce }(50-00-0) & \text { No } & \text { Yes } & \text { NA } \\ \text { Methyl Alcohol }(67-56-1) & \text { No } & \text { No } & \text { None } \\ \text { Water }(7732-18-5) & \text { No } & \text { No } & \text { None }\end{array}$

\section{Ecological Information}

Environmental Fate:

The following statements refer to the environmental fate of formaldehyde. When released into the soil, this material is expected to leach into groundwater. When released into water, this material is expected to readily biodegrade. When released into water, this material is not expected to evaporate significantly. This material is not expected to significantly bioaccumulate. When released into the air, this material is expected to be readily degraded by reaction with photochemically produced hydroxyl radicals. When released into the air, this material is expected to be readily degraded by photolysis. When released into the air, this material is expected to be readily removed from the atmosphere by dry and wet deposition. When released into the air, this material is expected to have a half-life of less than 1 day. The following statements refer to the environmental fate of methanol. When released into the soil, this material is expected to readily biodegrade. When released into the soil, this material is expected to leach into groundwater. When released into the soil, this material is expected to quickly evaporate. When released into water, this material is expected to readily biodegrade. When released into the water, this material is expected to have a half-life between 1 and 10 days. When released into the air, this material is expected to be readily degraded by reaction with photochemically produced hydroxyl radicals. When released into the air, this material is expected to be readily removed from the atmosphere by wet deposition. When released into air, this material is expected to have a half-life between 10 and 30 days.

Environmental Toxicity:

The following toxicity information is for the formaldehyde portion. This material is expected to be slightly toxic to aquatic life. The LC50/96-hour values for fish are between 10 and $100 \mathrm{mg} / \mathrm{l}$. The methanol portion is expected to be slightly toxic to aquatic life. The LC50/96-hour values for fish are between 10 and $100 \mathrm{mg} / 1$.

\section{Disposal Considerations}

Whatever cannot be saved for recovery or recycling should be handled as hazardous waste and sent to a RCRA approved incinerator or disposed in a RCRA approved waste facility. Processing, use or contamination of this product may change the waste management options. State and local disposal regulations may differ from federal disposal regulations. Dispose of container and unused contents in accordance with federal, state and local requirements.

\section{Transport Information}

Domestic (Land, D.O.T.)

Proper Shipping Name: RQ, FORMALDEHYDE, SOLUTION, FLAMMABLE 
Hazard Class: 3,8

UN/NA: UN1198

Packing Group: III

Information reported for product/size: $475 \mathrm{LB}$

International (Water, I.M.O.)

Proper Shipping Name: FORMALDEHYDE SOLUTIONS

Hazard Class: $3.3,8$

UN/NA: UN1198

Packing Group: III

Information reported for product/size: $475 \mathrm{LB}$

\section{Regulatory Information}

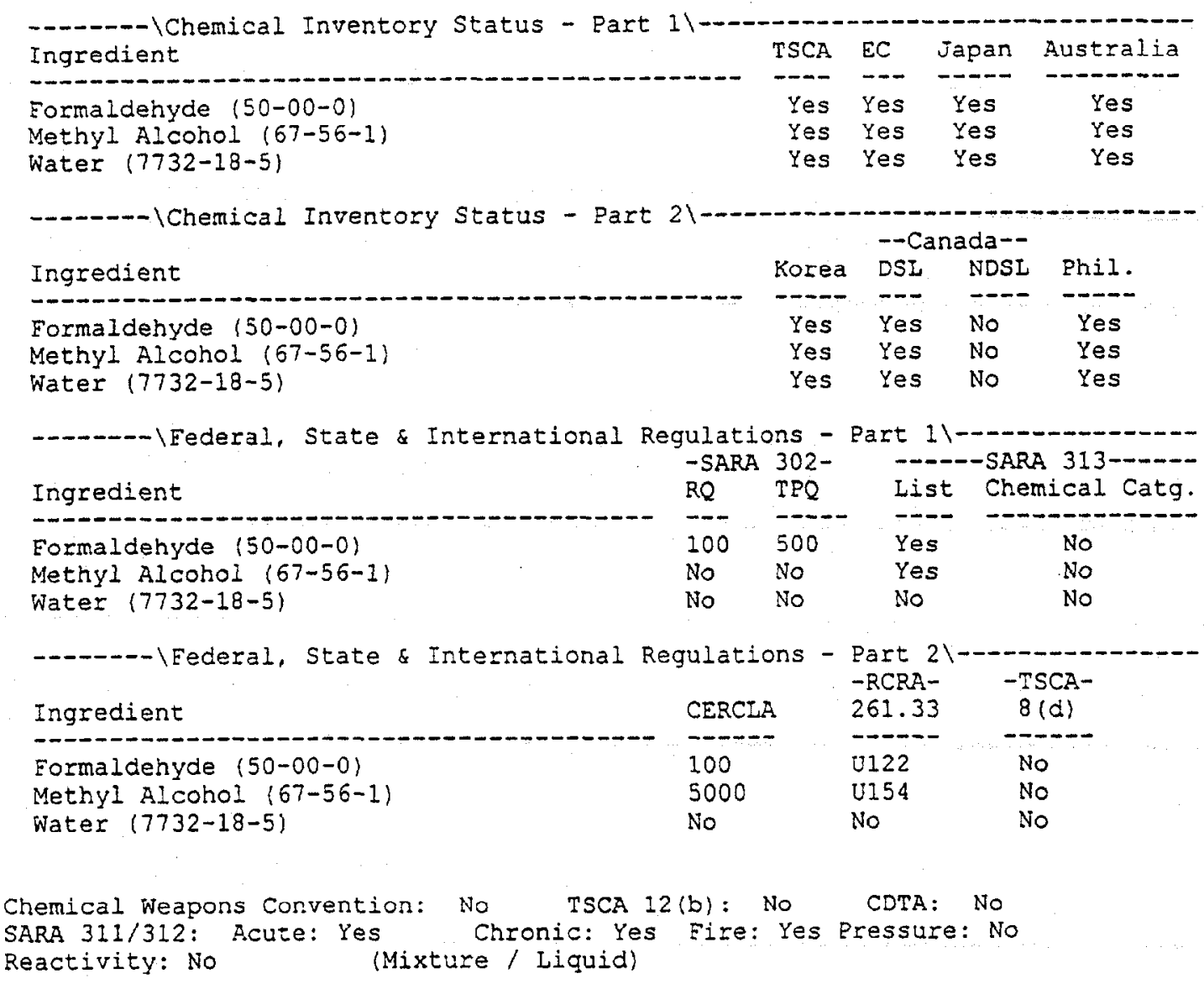

WARNING:

THIS PRODUCT CONTAINS A CHEMICAL(S) KNOWN TO THE STATE OF CALIFORNIA TO CAUSE CANCER.

Australian Hazchem Code: 2SE

Poison Schedule: S6

WHMIS:

This MSDS has been prepared according to the hazard criteria of the Controlled Products 
Regulations (CPR) and the MSDS contains all of the information required by the CPR.

\section{Other Information}

NFPA Ratings: Health: 3 Flammability: 2 Reactivity: 0

Label Hazard Warning:

POISON! DANGER! SUSPECT CANCER HAZARD. MAY CAUSE CANCER. Risk of cancer depends on level and duration of exposure. VAPOR HARMFUL. HARMFUL IF INHALED OR ABSORBED THROUGH SKIN. CAUSES IRRITATION TO SKIN, EYES AND RESPIRATORY TRACT. STRONG SENSITIZER. MAY BE FATAL OR CAUSE BLINDNESS IF SWALLOWED. CANNOT BE MADE NONPOISONOUS.

FLAMMABLE LIQUID AND VAPOR.

Label Precautions:

Keep away from heat, sparks and flame.

Do not get in eyes, on skin, or on clothing.

Do not breathe vapor.

Keep container closed.

Use only with adequate ventilation.

Wash thoroughly after handling.

Physical and health hazard information is available from employer and from material safety data sheets.

Label First Aid:

In all cases get medical attention immediately. If swallowed and the victim is conscious, dilute, inactivate, or absorb the ingested formaldehyde by giving milk, activated charcoal, or water. Any organic material will inactivate formaldehyde. Keep affected person warm and at rest. If vomiting occurs, keep head lower than hips. If inhaled, remove to fresh air. If not breathing, give artificial respiration. If breathing is difficult, give oxygen. In case of contact, immediately flush eyes or skin with plenty of water for at least 15 minutes while removing contaminated clothing and shoes. Wash clothing before reuse.

Product Use:

Laboratory Reagent.

Revision Information:

MSDS Section(s) changed since last revision of document include: $4,16$.

Disclaimer:

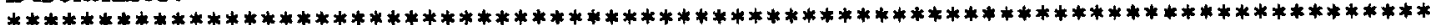

Mallinckrodt Baker, Inc. provides the information contained herein in good faith but makes no representation as to its comprehensiveness or accuracy. This document is intended only as a guide to the appropriate precautionary handling of the material by a properly trained person using this product. Individuals receiving the information must exercise their independent judgment in determining its appropriateness for a particular purpose. MALLINCKRODT BAKER, INC. MAKES NO REPRESENTATIONS OR WARRANTIES, EITHER EXPRESS OR IMPLIED, INCLUDING WITHOUT LIMITATION ANY WARRANTIES OF MERCHANTABILITY, FITNESS FOR A PARTICULAR PURPOSE WITH RESPECT TO THE INFORMATION SET FORTH HEREIN OR THE PRODUCT TO WHICH THE INFORMATION REFERS. ACCORDINGLY, MALLINCKRODT BAKER, INC. WILL NOT BE RESPONSIBLE FOR DAMAGES RESULTING FROM USE OF OR RELIANCE UPON THIS INFORMATION. 


\section{Material Safety Data Sheet}

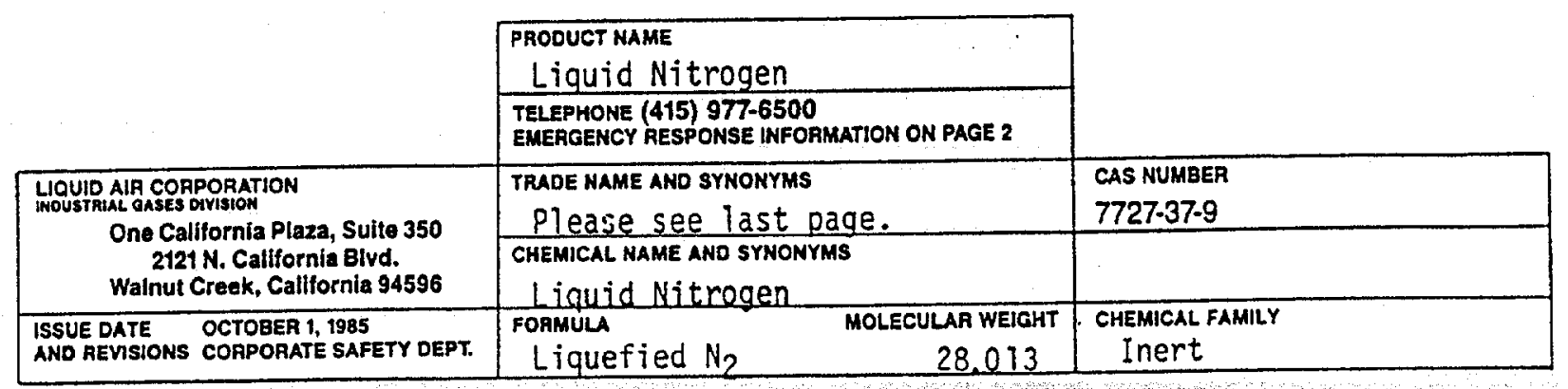

HEALTH HAZARD DATA (SEE NOTE ON LAST PAGE)

TMME WEIGHTED AVERAGE ExposuaE LIMIT Nitrogen is defined as a simple asphyxiant. oxygen levels should be maintained at greater than 18 molar percent at normal atmospheric pressure which is equivalent to a partial pressure of $135 \mathrm{~mm} \mathrm{Hg}$ (ACGIH, 1984-85).

SYMPTOMS OF EXPOSURE $\star$

Effects of exposure to high concentrations so as to displace the oxygen in air necessary for life may include any, all or none of the following:

o Loss of balance or dizziness;

0 Tightness in the frontal area of the forehead;

o Tingling of the tongue, fingertips or toes;

(Continued on last page.)

TOXICOLOGICAL PROPERTIES

Nitrogen is nontoxic but the liberation of a large amount in a confined area could displace the amount of oxygen in air necessary to support life.

Frostbite effects are a change in color of the skin to gray or white possibly followed by blistering.

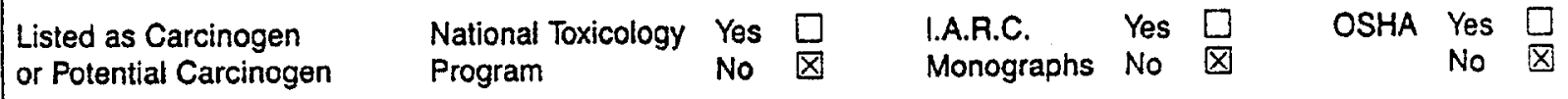

RECOMMENDED FIAST AID TREATMENT

PROMPT MEDICAL ATTENTION IS MANDATORY IN ALL CASES OF OVEREXPOSURE TO NITROGEN. RESCUE PERSONNEL SHOULD BE EQUIPPED WITH SELF-CONTAINED BREATHING APPARATUS.

Inhalation: Conscious persons should be assisted to an uncontaminated area and inhale fresh air. Quick removal from the contaminated area is most important. Unconscious persons should be moved to an uncontaminated area, given mouth-to-mouth resuscitation and supplemental oxygen. Medical assistance should be sought immediately.

For dermal contact or frostbite, flush affected areas with lukewarm water. DO NOT USE HOT WATER. A physician should see the patient promptly if the cryogenic "burn" has resulted in blistering of the dermal surface or deep tissue freezing.

Judgements as ro the suitability of inlormation herein for purchaser's purposes are necessarily purchaser's responsibility. Therelore, although reasonable care has been taken in the preparation of such

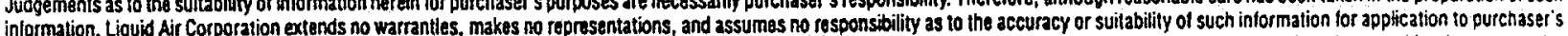
inlormation. Liquid Air Corporation extends no warrantles, makes no representations, and assumes no responsibility as to the accuracy or sultability of such intormation lor application lo purchaser's improper) use or application of the product. Oata Sheets may be changed from time to time. Be suse to consult tha latest edition.

Lic os 124 


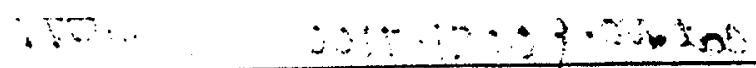

HAZARDOUS MIXTUAES OF OTHER LIQUIDS, SOLIDS, OR GASES
NONE

PHYSICAL DATA

\begin{tabular}{|c|c|}
\hline $\begin{array}{l}\text { BOILING POINT } \\
-320.445^{\circ} \mathrm{F} \quad\left(-195.803^{\circ} \mathrm{C}\right) \\
\end{array}$ & $\begin{array}{l}\text { LUOUIO DENSITY AT BOILING POINT } \\
50.48 \mathrm{lb} / \mathrm{ft}^{3} \quad\left(808.607 \mathrm{~kg} / \mathrm{m}^{3}\right)\end{array}$ \\
\hline $\begin{array}{l}\text { VAPOR PRESSURE } 70^{\circ} \mathrm{F}\left(21.1^{\circ} \mathrm{C}\right) \text { above the } \\
\text { critical temp. of }-232.51^{\circ} \mathrm{F}\left(-146.95^{\circ} \mathrm{C}\right)\end{array}$ & $\begin{array}{l}\text { GAS DENSITY AT 70 F 1 atm } \\
.07245 \mathrm{lb} / \mathrm{ft}^{3}\left(1.1605 \mathrm{~kg} / \mathrm{m}^{3}\right)\end{array}$ \\
\hline $\begin{array}{l}\text { SOLUBILIT IN WATER } \quad 068^{\circ} \mathrm{F} \quad\left(20^{\circ} \mathrm{C}\right) \text { Bunsen } \\
\text { coefficient }=.01557\end{array}$ & $\begin{array}{l}\text { FREEZING POINT } \\
-346.004^{\circ} \mathrm{F} \quad\left(-210.002^{\circ} \mathrm{C}\right)\end{array}$ \\
\hline $\begin{array}{l}\text { AppeaAAHCE AND OOOR } \\
\text { Clear, colorless, odorless liquid }\end{array}$ & \\
\hline
\end{tabular}

FIRE AND EXPLOSION HAZARD DATA

\begin{tabular}{|c|c|c|}
\hline $\begin{array}{l}\text { FLSH POINT (METHOD USED) } \\
N / A\end{array}$ & $\begin{array}{l}\text { AUTO IGNITION TEMPERATUAE } \\
N / A\end{array}$ & $\begin{array}{l}\text { FLAMMABLE LIMITS * BY VOLUME } \\
\text { N/A }\end{array}$ \\
\hline $\begin{array}{l}\text { EXTINGUISHING MEDIA } \\
\text { Nonflammable, inert }\end{array}$ & 4 & \begin{tabular}{c|c} 
ELECTRICAL CuSSIFICATION \\
Nonhazardous
\end{tabular} \\
\hline $\begin{array}{l}\text { SPECIAL FIRE FIGHTING PROCEDURES } \\
\text { N/A }\end{array}$ & & \\
\hline $\begin{array}{l}\text { UNUSUAL FIRE AND EXPLOSION HAZARD } \\
\text { N/A }\end{array}$ & & \\
\hline
\end{tabular}

REACTIVITY DATA

\begin{tabular}{|c|c|c|}
\hline $\begin{array}{l}\text { STABILIT } \\
\text { Unalable }\end{array}$ & & CONDITIONS TO AVOIO \\
\hline Stable & $x$ & \\
\hline \multicolumn{3}{|c|}{ INCOMPATIBILITY (Matorlats to avold) } \\
\hline \multicolumn{3}{|c|}{$\begin{array}{l}\text { HAZARDOUS DECOMPOSITION PRODUCTS } \\
\text { None }\end{array}$} \\
\hline \multicolumn{2}{|c|}{$\begin{array}{l}\text { HAZARDOUS POLYMEAIZATION } \\
\text { MaY OCEU }\end{array}$} & CONDITIONS TO AVOID \\
\hline Will Not Oceur & $x$ & \\
\hline
\end{tabular}

SPILL OR LEAK PROCEDURES

STEPS TO BE TAKEN IN CASE MATERIAL IS RELEASED OR SPILLEO

See note on last page.

WASTE DISPOSAL METHOD

See note on last page. 


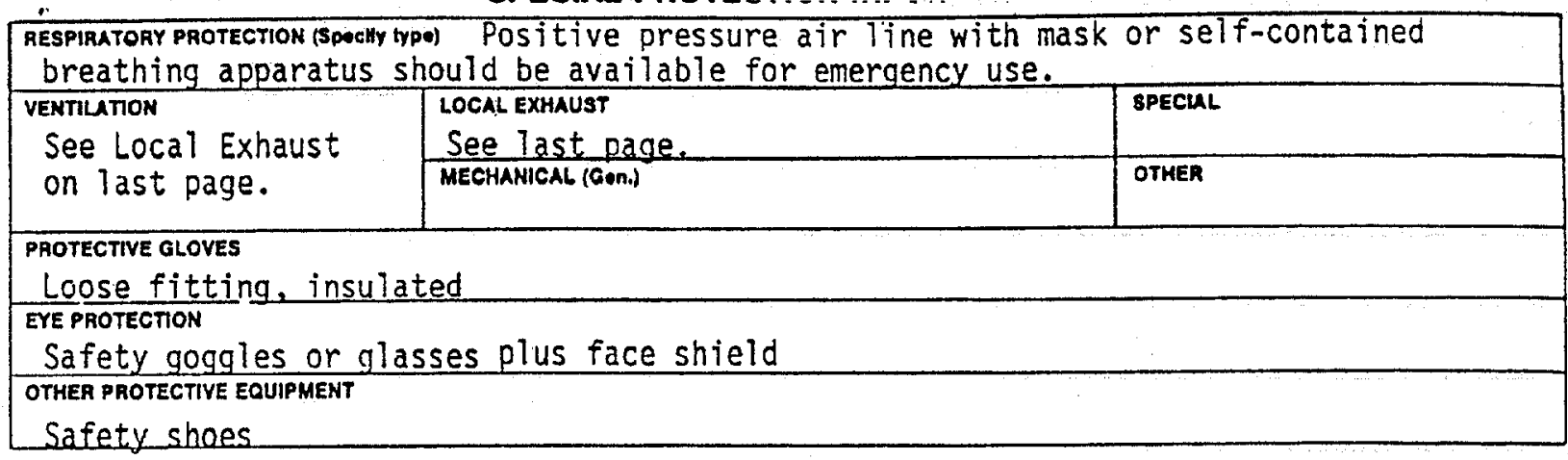

\section{SPECIAL PRECAUTIONS*}

SPECIAL LABELNG INFOAMATON Nitrogen, refrigerated liquid (cryogenic liquid) I.D. No.: UN 1977
DOT Shipping Name: NoT Shipping Label: Nonflammable Gas
DOT OOT Hazard Class: Nonflammable gas
SPECIAL HANDUNG RECOMMENDATIONS

See note on last page re Spill or Leak Procedures. Also see CGA Safety Bulletin $\mathrm{SB}-2$ and $\mathrm{CGA}$ pamphlets $\mathrm{P}-9, \mathrm{P}-12$ and $\mathrm{P}-14$.

SB-2 Oxygen Deficient Atmospheres

P-9 The Inert Gases - Argon, Nitrogen and Helium

P-12 Safe Handling of Cryogenic Liquids

P-14 Accident Prevention in Oxygen-Rich and Oxygen-Deficient Atmospheres

For additional handling recommendatlons consult L'Air Liquide's Encyclopedia de Gaz or Compressed Gas Association Pamphlet P-1.

\section{SPECIAL STORAGE RECOMMENDATIONS}

See note on last page re Spill or Leak Procedures. Also see CGA Safety Bulletin SB-2 and CGA pamphlets $\mathrm{P}-9, \mathrm{P}-12$ and $\mathrm{P}-14$.

Do not store cylinders in sub-surface or closed (poorly ventilated) areas. Nitrogen gas can cause suffocation without waming.

For additional storage recommendations consult L'Air Liquide's Encyclopedia de Gaz or Compressed Gas Assoclatlon Pamphle! P-1. SPECIAL PACKCGING RECOMMENDATIONS

Liquid nitrogen cannot be handled in carbon or low alloy steels. Eighteen-eight and 18-10 stainless steels are acceptable as are copper and its alloys, nickel and its alloys, brass, bronze, silicon alloys, Mone ${ }^{Q}$, Incone ${ }^{\circ}$, and beryllium. Also see CGA Safety Bulletin SB-2 and CGA pamphlets $P-9$ and P-12.

\section{OTHER RECOMMENDATIONS OR PRECAUTIONS}

Liquefied gas cylinders should not be refilled except by qualified producers of these products. Shipment of a compressed gas container which has not been filled by the owner or with his (written) consent is a violation of Federal Law (49CFR).

tVarious Government agencles (i.e. Department of Transportation, Occupational Safety and Health Administration, Food snd Drug Administration and others) may have specific regulations concerning the transportation, handling, storage or use of this product which may not be contained herein. The customer or user of this product should be tamiliar with these regutations. 
TRADE NAME AND SYNONYMS: (Continued)

Liquid Nitrogen; LIN; Nitrogen, refrigerated liquid (cryogenic liquid)

HEALTH HAZARD DATA: (Continued)

Note: Except where specified, the heal th hazard data and most of the other data in this material safety data sheet are for gaseous nitrogen. One volume of liquid nitrogen at its boiling point and atmospheric pressure will vaporize into approximately 695 volumes of gaseous nitrogen at $70^{\circ} \mathrm{F}\left(21.1^{\circ} \mathrm{C}\right)$ and 1 atmosphere.

SYMPTOMS OF EXPOSURE: (Continued)

o Weakened speech leading to the inability to utter sounds;

0 Rapid reduction in the ability to perform movements;

- Reduced consciousness of the surroundings;

o Loss of tactile sensations;

o Heightened mental activity.

It should be recognized that it is possible that none of the above symptoms may occur in nitrogen asphyxia so that there are no definite warning symptoms.

Contact with the cryogenic liquid or cold piping containing the liquid can cause tissue freezing or frostbite on dermal contact or if splashed into the eyes.

* For additional information, refer to L'Air Liquide's Encyclopedie des Gaz.

HOTE re SPILL OR LEAK PROCEDURES:

Liquid nitrogen is delivered to a customer into stationary vacuum-jacketed vessels at the customer's location or in portable vacuum-jacketed "liquid" cylinders.

Stationary customer-site vessels should be operated in accordance with the manufacturer's and Liquid Air Corporation's instructions. Do not attempt to repair, adjust, or in any other way modify the operation of these vessels. If there is a malfunction or other type of operational problem with the vessel, contact the closest Liquid Air Corporation location immediately.

Liquid nitrogen cylinders should be used only in well-ventilated areas and in accordance with the manufacturer's and Liquid Air Corporation's instructions. These cylinders must always be kept in an upright position. Specialized hand trucks are needed for their movement. A "first in-first out" inventory system should be used with these cylinders.

\section{LOCAL EXHAUST: (Continued)}

To prevent accumulation of high concentrations so as to reduce the oxygen level in the air to less than 13 molar percent. 


\section{Appendix C}

HEALTH AND SAFETY PLAN ACCEPTANCE AND TRAINING ACKNOWLEDGEMENT 
Instructions: This form is to be completed by each person that works on the NABIR Field Research Center work sites and returned to the Site Safety and Health Officer.

I have read and agree to abide by the contents of the SITE-SPECIFIC HEALTH AND SAFETY PLAN for work activities at the NABIR Field Research Center. I have completed the training requirements specified in the plan. I am currently participating in a medical surveillance program that satisfies the requirements of CFR 1910.120.

Signature:

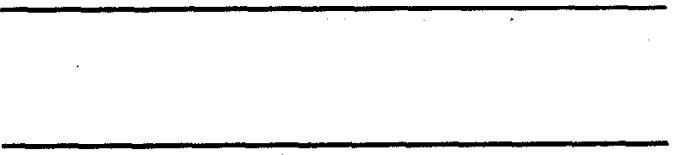

Date:

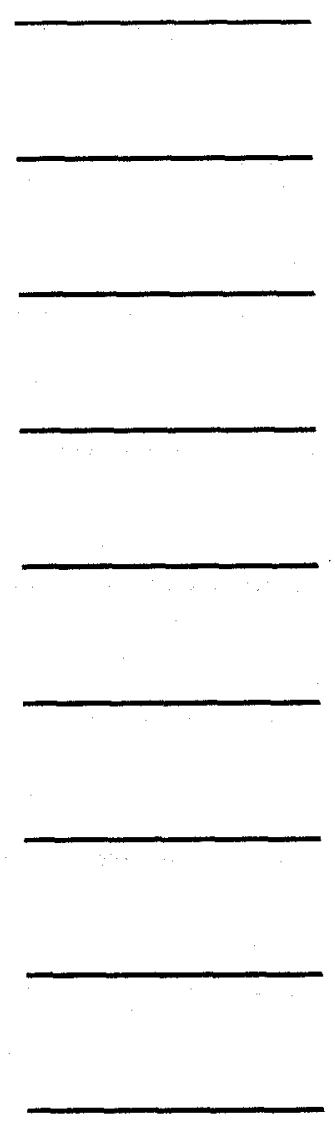

\section{Return to:}

FRC Site Safety and Health Officer or FRC Field Manager at FRC Field Site Trailer in Y-12 Bear Creek Valley or ORNL Building 1505, Room 302

P.O. Box 2008 MS 6038

Oak Ridge, TN 37831-6038 